\title{
Atmospheric Ring-Closure and Dehydration Reactions of 1,4-Hydroxycarbonyls in the Gas Phase: The Impact of Catalysts
}

Parandaman Arathala, Chanin B. Tangtartharakul, and Amitabha Sinha*

Department of Chemistry and Biochemistry, University of California-San Diego, La Jolla, California 92093

\section{Supporting Information}

This section contains: Table S1-S9 contains total electronic energies including zero-point energy correction at different levels of theory, optimized geometries of reactants, products, complexes, and transition states with their z-matrices, vibrational frequencies, rotational constants, imaginary frequencies of all the transition states, L-J parameters, bimolecular rate constants in presence and absence of tunneling, Figure S1-S4 shows structures of various higher energy TSs, two conformers of sulfuric acid, and the PES diagrams for the cyclization of 4-OH-BL catalyzed by cis- $\mathrm{H}_{2} \mathrm{SO}_{4}$ and 2-OH-THF + OH reaction. 
Table S1: Calculated total electronic energies for monomers, their complexes, and transition states (TS) at the M06-2X, and CCSD(T) levels. Zero point energy (ZPE) corrections are given at the M06-2X level.

\begin{tabular}{|c|c|c|c|}
\hline Species & M06-2X/6-311++G(3df,3pd) & ZPE (M06-2X) & CCSD(T)/6-311++G(3df,3pd) \\
\hline $\mathrm{HOCH}_{2} \mathrm{CH}_{2} \mathrm{CH}_{2} \mathrm{CHO}(4-\mathrm{OH}-\mathrm{BL})$ & -307.653841 & 0.119841 & -307.1975777 \\
\hline $\mathrm{H}_{2} \mathrm{O}$ & -76.427007 & 0.021655 & -76.3374558 \\
\hline $\mathrm{HCOOH} \mathrm{(FA)}$ & -189.763594 & 0.034421 & -189.5061194 \\
\hline $\mathrm{HO}_{2}$ & -150.901958 & 0.014595 & -150.7129418 \\
\hline $\mathrm{HOCH}_{2} \mathrm{CH}_{2} \mathrm{CH}_{2} \mathrm{CHO}(\mathrm{TSa})$ & -307.595345 & 0.117299 & -307.1395467 \\
\hline 2-OH-THF $\left(\mathrm{H}_{8} \mathrm{C}_{4} \mathrm{O}_{2}\right)$ & -307.665582 & 0.123073 & -307.2081492 \\
\hline 2-OH-THF (TSb) & -307.554791 & 0.114209 & -307.0988683 \\
\hline 2-OH-THF (PC) & -307.638096 & 0.118263 & -307.1821994 \\
\hline 2,3-Dihydrofuran & -231.202978 & 0.093459 & -230.8375675 \\
\hline $\mathrm{HOCH}_{2} \mathrm{CH}_{2} \mathrm{CH}_{2} \mathrm{CHO} \cdot \bullet \mathrm{H}_{2} \mathrm{O}(\mathrm{RC} 1)$ & -384.09707 & 0.145508 & -383.5507052 \\
\hline $\mathrm{HOCH}_{2} \mathrm{CH}_{2} \mathrm{CH}_{2} \mathrm{CHO} \bullet \cdot \mathrm{H}_{2} \mathrm{O}(\mathrm{TS} 1)$ & -384.06283 & 0.142429 & -383.5134353 \\
\hline $\mathrm{HOCH}_{2} \mathrm{CH}_{2} \mathrm{CH}_{2} \mathrm{CHO} \bullet \cdot \mathrm{H}_{2} \mathrm{O}(\mathrm{PC} 1)$ & -384.107719 & 0.148530 & -383.5603564 \\
\hline 2-OH-THF•• $\mathrm{H}_{2} \mathrm{O}(\mathrm{RC} 2)$ & -384.104224 & 0.148456 & -383.556510 \\
\hline $2-\mathrm{OH}-\mathrm{THF} \cdot \bullet \mathrm{H}_{2} \mathrm{O}(\mathrm{TS} 2)$ & -384.013461 & 0.139410 & -383.465203 \\
\hline $2-\mathrm{OH}-\mathrm{THF} \cdot \bullet \mathrm{H}_{2} \mathrm{O}(\mathrm{PC} 2)$ & -384.077728 & 0.144000 & -383.532061 \\
\hline $\mathrm{HOCH}_{2} \mathrm{CH}_{2} \mathrm{CH}_{2} \mathrm{CHO} \bullet \bullet \mathrm{H}_{2} \mathrm{O}(\mathrm{RC} 1 \mathrm{~A})$ & -384.097071 & 0.145514 & -383.550706 \\
\hline $\mathrm{HOCH}_{2} \mathrm{CH}_{2} \mathrm{CH}_{2} \mathrm{CHO} \cdot \bullet \mathrm{H}_{2} \mathrm{O}(\mathrm{TS} 1 \mathrm{~A})$ & -384.062855 & 0.142301 & -383.513440 \\
\hline $\mathrm{HOCH}_{2} \mathrm{CH}_{2} \mathrm{CH}_{2} \mathrm{CHO} \bullet \mathrm{H}_{2} \mathrm{O}(\mathrm{PC} 1 \mathrm{~A})$ & -384.107718 & 0.148507 & -383.5603606 \\
\hline $\mathrm{HOCH}_{2} \mathrm{CH}_{2} \mathrm{CH}_{2} \mathrm{CHO} \cdot \bullet \mathrm{HO}_{2}(\mathrm{RC} 3)$ & -458.578737 & 0.138044 & -457.9327236 \\
\hline $\mathrm{HOCH}_{2} \mathrm{CH}_{2} \mathrm{CH}_{2} \mathrm{CHO} \bullet \bullet \mathrm{HO}_{2}(\mathrm{TS} 3)$ & -458.560440 & 0.135496 & -457.9119625 \\
\hline $\mathrm{HOCH}_{2} \mathrm{CH}_{2} \mathrm{CH}_{2} \mathrm{CHO} \bullet \cdot \mathrm{HO}_{2}(\mathrm{PC} 3)$ & -458.587776 & 0.140778 & -457.9406635 \\
\hline $2-\mathrm{OH}-\mathrm{THF} \cdot \bullet \mathrm{HO}_{2}(\mathrm{RC} 4)$ & -458.585734 & 0.140864 & -457.9389513 \\
\hline $2-\mathrm{OH}-\mathrm{THF} \bullet \bullet \mathrm{HO}_{2}(\mathrm{TS} 4)$ & -458.517352 & 0.134294 & -457.8714463 \\
\hline $2-\mathrm{OH}-\mathrm{THF} \cdot \bullet \mathrm{HO}_{2}(\mathrm{PC} 4)$ & -458.563414 & 0.136379 & -457.918513 \\
\hline $\mathrm{HOCH}_{2} \mathrm{CH}_{2} \mathrm{CH}_{2} \mathrm{CHO} \bullet \mathrm{HO}_{2}(\mathrm{RC} 3 \mathrm{~A})$ & -458.575703 & 0.137942 & \\
\hline $\mathrm{HOCH}_{2} \mathrm{CH}_{2} \mathrm{CH}_{2} \mathrm{CHO} \cdot \bullet \mathrm{HO}_{2}(\mathrm{TS} 3 \mathrm{~A})$ & -458.559911 & 0.135507 & \\
\hline
\end{tabular}




\begin{tabular}{|c|c|c|c|}
\hline $\mathrm{HOCH}_{2} \mathrm{CH}_{2} \mathrm{CH}_{2} \mathrm{CHO} \bullet \cdot \mathrm{HO}_{2}(\mathrm{PC} 3 \mathrm{~A})$ & -458.591012 & 0.141046 & \\
\hline $\mathrm{HOCH}_{2} \mathrm{CH}_{2} \mathrm{CH}_{2} \mathrm{CHO} \bullet \mathrm{FA}(\mathrm{RC} 5)$ & -497.437991 & 0.156911 & -496.7254278 \\
\hline $\mathrm{HOCH}_{2} \mathrm{CH}_{2} \mathrm{CH}_{2} \mathrm{CHO} \bullet \cdot \mathrm{FA}$ (TS5) & -497.423185 & 0.153784 & -496.7074360 \\
\hline $\mathrm{HOCH}_{2} \mathrm{CH}_{2} \mathrm{CH}_{2} \mathrm{CHO} \bullet \cdot \mathrm{FA}(\mathrm{PC} 5)$ & -497.452754 & 0.160158 & -496.7387506 \\
\hline $2-\mathrm{OH}-\mathrm{THF} \bullet$ FA (RC6) & -497.444519 & 0.159815 & -496.7297783 \\
\hline $2-\mathrm{OH}-\mathrm{THF} \bullet \cdot \mathrm{FA}(\mathrm{TS} 6)$ & -497.377689 & 0.152547 & -496.6644500 \\
\hline 2-OH-THF••FA (PC6) & -497.424826 & 0.155780 & -496.7106299 \\
\hline $\mathrm{HOCH}_{2} \mathrm{CH}_{2} \mathrm{CH}_{2} \mathrm{CHO} \bullet \mathrm{FA}(\mathrm{RC} 5 \mathrm{~A})$ & -497.439215 & 0.157062 & -496.7256896 \\
\hline $\mathrm{HOCH}_{2} \mathrm{CH}_{2} \mathrm{CH}_{2} \mathrm{CHO} \bullet \mathrm{FA}(\mathrm{TS} 5 \mathrm{~A})$ & -497.421223 & 0.154075 & -496.7048414 \\
\hline $\mathrm{HOCH}_{2} \mathrm{CH}_{2} \mathrm{CH}_{2} \mathrm{CHO} \bullet \cdot \mathrm{FA}(\mathrm{PC} 5 \mathrm{~A})$ & -497.451405 & 0.160292 & -496.7359412 \\
\hline $\mathrm{HNO}_{3}$ & -280.887216 & 0.027522 & -280.523669 \\
\hline $\mathrm{HOCH}_{2} \mathrm{CH}_{2} \mathrm{CH}_{2} \mathrm{CHO} \cdot \bullet \mathrm{HNO}_{3}(\mathrm{RC} 7)$ & -588.561723 & 0.149578 & -587.742064 \\
\hline $\mathrm{HOCH}_{2} \mathrm{CH}_{2} \mathrm{CH}_{2} \mathrm{CHO} \cdot \bullet \mathrm{HNO}_{3}$ (TS7) & -588.549210 & 0.147301 & -587.726562 \\
\hline $\mathrm{HOCH}_{2} \mathrm{CH}_{2} \mathrm{CH}_{2} \mathrm{CHO} \cdot \bullet \mathrm{HNO}_{3}(\mathrm{PC} 7)$ & -588.577173 & 0.152701 & -587.7559366 \\
\hline $2-\mathrm{OH}-\mathrm{THF} \bullet \cdot \mathrm{HNO}_{3}(\mathrm{RC} 8)$ & -588.572268 & 0.152368 & -587.7508618 \\
\hline 2-OH-THF••-HNO ${ }_{3}$ (TS8) & -588.511201 & 0.144005 & -587.6906286 \\
\hline 2-OH-THF••HNO $\mathrm{HN}_{3}(\mathrm{PC} 8)$ & -588.551079 & 0.148246 & -587.7312269 \\
\hline $\mathrm{HOCH}_{2} \mathrm{CH}_{2} \mathrm{CH}_{2} \mathrm{CHO} \cdot \bullet \mathrm{HNO}_{3}(\mathrm{TS} 7 \mathrm{~A})$ & -588.548993 & 0.148238 & -587.7257884 \\
\hline $\mathrm{HOCH}_{2} \mathrm{CH}_{2} \mathrm{CH}_{2} \mathrm{CHO} \cdot \bullet \mathrm{HNO}_{3}(\mathrm{PC} 7 \mathrm{~A})$ & -588.576177 & 0.152869 & -587.7539755 \\
\hline trans- $\mathrm{H}_{2} \mathrm{SO}_{4}$ & -700.288805 & 0.040328 & -699.4493017 \\
\hline $\mathrm{HOCH}_{2} \mathrm{CH}_{2} \mathrm{CH}_{2} \mathrm{CHO} \bullet \bullet$ trans- $\mathrm{H}_{2} \mathrm{SO}_{4}(\mathrm{RC} 9)$ & -1007.971795 & 0.161860 & -1006.675801 \\
\hline $\mathrm{HOCH}_{2} \mathrm{CH}_{2} \mathrm{CH}_{2} \mathrm{CHO} \bullet \bullet$ trans- $\mathrm{H}_{2} \mathrm{SO}_{4}$ (TS9) & -1007.966371 & 0.162161 & -1006.669737 \\
\hline $\mathrm{HOCH}_{2} \mathrm{CH}_{2} \mathrm{CH}_{2} \mathrm{CHO} \cdot$ trans- $\mathrm{H}_{2} \mathrm{SO}_{4}(\mathrm{PC} 9)$ & -1007.980384 & 0.164925 & -1006.683624 \\
\hline 2-OH-THF••trans- $\mathrm{H}_{2} \mathrm{SO}_{4}(\mathrm{RC} 10)$ & -1007.975786 & 0.164792 & -1006.679955 \\
\hline 2-OH-THF••trans- $\mathrm{H}_{2} \mathrm{SO}_{4}(\mathrm{TS} 10)$ & -1007.929235 & 0.156166 & -1006.63560 \\
\hline $2-\mathrm{OH}-\mathrm{THF} \bullet \bullet$ trans $-\mathrm{H}_{2} \mathrm{SO}_{4}(\mathrm{PC} 10)$ & -1007.956692 & 0.160591 & -1006.662076 \\
\hline $\mathrm{HOCH}_{2} \mathrm{CH}_{2} \mathrm{CH}_{2} \mathrm{CHO} \bullet \bullet$ trans $-\mathrm{H}_{2} \mathrm{SO}_{4}(\mathrm{RC} 9 \mathrm{~A})$ & -1007.968713 & 0.162286 & \\
\hline $\mathrm{HOCH}_{2} \mathrm{CH}_{2} \mathrm{CH}_{2} \mathrm{CHO} \bullet \bullet$ trans $-\mathrm{H}_{2} \mathrm{SO}_{4}(\mathrm{TS} 9 \mathrm{~A})$ & -1007.961176 & 0.160249 & \\
\hline $\mathrm{HOCH}_{2} \mathrm{CH}_{2} \mathrm{CH}_{2} \mathrm{CHO} \bullet \bullet$ trans- $\mathrm{H}_{2} \mathrm{SO}_{4}(\mathrm{PC} 9 \mathrm{~A})$ & -1007.983621 & 0.165175 & \\
\hline cis- $\mathrm{H}_{2} \mathrm{SO}_{4}$ & -700.286728 & 0.039994 & -699.4471971 \\
\hline $\mathrm{HOCH}_{2} \mathrm{CH}_{2} \mathrm{CH}_{2} \mathrm{CHO} \bullet \bullet_{\text {cis- }} \mathrm{H}_{2} \mathrm{SO}_{4}(\mathrm{RC} 9 \mathrm{~B})$ & -1007.972142 & 0.162077 & -1006.676444 \\
\hline $\mathrm{HOCH}_{2} \mathrm{CH}_{2} \mathrm{CH}_{2} \mathrm{CHO} \cdot \bullet_{\text {cis- }} \mathrm{H}_{2} \mathrm{SO}_{4}$ (TS9B) & -1007.966539 & 0.161942 & -1006.670089 \\
\hline
\end{tabular}




\begin{tabular}{|c|c|c|c|}
\hline $\mathrm{HOCH}_{2} \mathrm{CH}_{2} \mathrm{CH}_{2} \mathrm{CHO} \cdot{ }^{\circ}$ cis- $\mathrm{H}_{2} \mathrm{SO}_{4}$ (PC9B) & -1007.980676 & 0.164916 & -1006.683835 \\
\hline OH radical & -75.730338 & 0.008600 & -75.6398533 \\
\hline 2-OH-THF••OH (TS11) & -383.397036 & 0.130532 & -382.8485364 \\
\hline 2-OH-THF・•OH (RC11) & -383.410228 & 0.133892 & -382.8616454 \\
\hline 2-OH-THF••OH (PC11) & -383.447023 & 0.135044 & -382.8965446 \\
\hline 2-OH-THF••OH (P11) & -307.005014 & 0.109507 & -306.5446547 \\
\hline $2-\mathrm{OH}-\mathrm{THF} \bullet \mathrm{OH}(\mathrm{TS} 12)$ & -383.390773 & 0.128224 & -382.8387363 \\
\hline 2-OH-THF••OH (RC12) & -383.406234 & 0.134861 & -382.856220 \\
\hline 2-OH-THF••OH (PC12) & -383.425740 & 0.133476 & -382.8752424 \\
\hline 2-OH-THF••OH (P12) & -306.988091 & 0.109185 & -306.5287700 \\
\hline 2-OH-THF••OH (TS13) & -383.407669 & 0.129903 & -382.8455042 \\
\hline 2-OH-THF・•OH (RC13) & -383.407664 & 0.134448 & -382.8584965 \\
\hline 2-OH-THF••OH (PC13) & -383.435329 & 0.132724 & -382.885576 \\
\hline 2-OH-THF••OH (P13) & -306.997722 & 0.108069 & -306.5376519 \\
\hline 2-OH-THF••OH (TS14) & -383.395859 & 0.130187 & -382.8470433 \\
\hline 2-OH-THF••OH (RC14) & -383.409049 & 0.135075 & -382.8587668 \\
\hline 2-OH-THF••OH (PC14) & -383.437713 & 0.133379 & -382.8869497 \\
\hline 2-OH-THF••OH (TS15) & -383.392117 & 0.129761 & -382.844020 \\
\hline 2-OH-THF••OH (RC15) & -383.399334 & 0.132598 & -382.849939 \\
\hline 2-OH-THF••OH (PC15) & -383.436693 & 0.132450 & -382.885930 \\
\hline 2-OH-THFं (P14) & -306.999984 & 0.107876 & -306.5398823 \\
\hline 2-OH-THF••OH (TS16) & -383.391145 & 0.129549 & -382.8430873 \\
\hline 2-OH-THF••OH (PC16) & -383.437864 & 0.132948 & -382.8869942 \\
\hline 2-OH-THF••OH (TS17) & -383.397412 & 0.130653 & -382.8488396 \\
\hline 2-OH-THF••OH (PC17) & -383.443000 & 0.133132 & -382.893255 \\
\hline 2-OH-THF $(\mathrm{P} 15)$ & -307.005940 & 0.108805 & -306.5462665 \\
\hline $2-\mathrm{OH}-\mathrm{THF} \bullet \mathrm{OH}(\mathrm{TS} 18)$ & -383.400772 & 0.131357 & -382.8521174 \\
\hline 2-OH-THF••OH (PC18) & -383.443943 & 0.134190 & -382.893391 \\
\hline $\mathrm{H}_{2} \mathrm{O}$ & -76.427009 & 0.021667 & -76.3374354 \\
\hline $\mathrm{O}_{2}$ & -150.318893 & 0.004052 & -150.1276633 \\
\hline $2-\mathrm{OH}-\mathrm{THF} \bullet \bullet \mathrm{O}_{2}\left(\mathrm{RO}_{2}\right)$ & -457.386984 & 0.119386 & -456.735370 \\
\hline $2-\mathrm{OH}-\mathrm{THF}^{*} \bullet \mathrm{O}_{2}(\mathrm{TS} 19)$ & -457.337209 & 0.11225 & -456.687323 \\
\hline
\end{tabular}




\begin{tabular}{|l|l|l|l|}
\hline $2-\mathrm{OH}-\mathrm{THF}^{\bullet} \bullet \mathrm{O}_{2}(\mathrm{PC} 19)$ & -457.363843 & 0.112849 & -456.716936 \\
\hline $2-\mathrm{OH}-\mathrm{THF}^{\bullet} \bullet \mathrm{O}_{2}$ (TS20) & -457.331745 & 0.112883 & -456.682022 \\
\hline $2-\mathrm{OH}-\mathrm{THF}^{\bullet} \bullet \mathrm{O}_{2}(\mathrm{PC} 20)$ & -457.360671 & 0.116505 & -456.709527 \\
\hline $2,3-\mathrm{Dihydro}-2$-furanol & -306.441099 & 0.098753 & -305.979768 \\
\hline Succinaldehyde & -306.443592 & 0.095024 & -305.986033 \\
\hline $\mathrm{HO}_{2}$ & -150.901958 & 0.014595 & -150.712941 \\
\hline
\end{tabular}


Table S2: Imaginary frequencies of all transition states at the MO6-2X/6-311++g(3df,3pd) levels of calculation.

\begin{tabular}{|c|c|}
\hline & imaginary freq \\
\hline Transition State & MO6-2X/6-311++g(3df,3pd) \\
\hline $\mathrm{HOCH}_{2} \mathrm{CH}_{2} \mathrm{CH}_{2} \mathrm{CHO}$ (TSa) & 1517 \\
\hline $\mathrm{HOCH}_{2} \mathrm{CH}_{2} \mathrm{CH}_{2} \mathrm{CHO}(\mathrm{TSb})$ & 1800 \\
\hline $\mathrm{HOCH}_{2} \mathrm{CH}_{2} \mathrm{CH}_{2} \mathrm{CHO} \cdot \bullet \mathrm{H}_{2} \mathrm{O}(\mathrm{TS} 1)$ & 1166 \\
\hline $2-\mathrm{OH}-\mathrm{THF} \bullet \bullet \mathrm{H}_{2} \mathrm{O}(\mathrm{TS} 2)$ & 1469 \\
\hline $\mathrm{HOCH}_{2} \mathrm{CH}_{2} \mathrm{CH}_{2} \mathrm{CHO} \cdot \bullet \mathrm{H}_{2} \mathrm{O}(\mathrm{TS} 1 \mathrm{~A})$ & 1157 \\
\hline $\mathrm{HOCH}_{2} \mathrm{CH}_{2} \mathrm{CH}_{2} \mathrm{CHO} \cdot \bullet \mathrm{HO}_{2}(\mathrm{TS} 3)$ & 700 \\
\hline 2-OH-THF•• $\mathrm{HO}_{2}(\mathrm{TS} 4)$ & 262 \\
\hline $\mathrm{HOCH}_{2} \mathrm{CH}_{2} \mathrm{CH}_{2} \mathrm{CHO} \cdot \bullet \mathrm{HO}_{2}(\mathrm{TS} 3 \mathrm{~A})$ & 600 \\
\hline $\mathrm{HOCH}_{2} \mathrm{CH}_{2} \mathrm{CH}_{2} \mathrm{CHO} \cdot \bullet \mathrm{FA}$ (TS5) & 642 \\
\hline 2-OH-THF••FA (TS6) & 724 \\
\hline $\mathrm{HOCH}_{2} \mathrm{CH}_{2} \mathrm{CH}_{2} \mathrm{CHO} \cdot \bullet \mathrm{HNO}_{3}$ (TS7) & 532 \\
\hline 2-OH-THF•• $\mathrm{HNO}_{3}$ (TS8) & 475 \\
\hline $\mathrm{HOCH}_{2} \mathrm{CH}_{2} \mathrm{CH}_{2} \mathrm{CHO} \cdot \bullet \mathrm{HNO}_{3}(\mathrm{TS} 7 \mathrm{~A})$ & 369 \\
\hline $\mathrm{HOCH}_{2} \mathrm{CH}_{2} \mathrm{CH}_{2} \mathrm{CHO} \cdot \bullet$ trans- $\mathrm{H}_{2} \mathrm{SO}_{4}$ (TS9) & 196 \\
\hline 2-OH-THF••trans- $\mathrm{H}_{2} \mathrm{SO}_{4}(\mathrm{TS} 10)$ & 953 \\
\hline $\mathrm{HOCH}_{2} \mathrm{CH}_{2} \mathrm{CH}_{2} \mathrm{CHO} \bullet \bullet$ trans $-\mathrm{H}_{2} \mathrm{SO}_{4}(\mathrm{TS} 9 \mathrm{~A})$ & 367 \\
\hline $\mathrm{HOCH}_{2} \mathrm{CH}_{2} \mathrm{CH}_{2} \mathrm{CHO} \bullet \bullet^{\circ}$ is $-\mathrm{H}_{2} \mathrm{SO}_{4}$ (TS9B) & 209 \\
\hline 2-OH-THF • OH (TS11) & 660 \\
\hline 2-OH-THF••OH (TS12) & 1592 \\
\hline 2-OH-THF••OH (TS13) & 1026 \\
\hline 2-OH-THF••OH (TS14) & 1006 \\
\hline
\end{tabular}




\begin{tabular}{|l|c|}
\hline $2-\mathrm{OH}-\mathrm{THF} \bullet \cdot \mathrm{OH}(\mathrm{TS} 15)$ & 539 \\
\hline $2-\mathrm{OH}-\mathrm{THF} \bullet \cdot \mathrm{OH}(\mathrm{TS} 16)$ & 623 \\
\hline $2-\mathrm{OH}-\mathrm{THF} \cdot \bullet \mathrm{OH}(\mathrm{TS} 17)$ & 877 \\
\hline $2-\mathrm{OH}-\mathrm{THF} \cdot \bullet \mathrm{OH}(\mathrm{TS} 18)$ & 674 \\
\hline $2-\mathrm{OH}-\mathrm{THF} \cdot \bullet \mathrm{O}_{2}(\mathrm{TS} 19)$ & 1124 \\
\hline $2-\mathrm{OH}-\mathrm{THF} \cdot \bullet \mathrm{O}_{2}(\mathrm{TS} 20)$ & \\
\hline
\end{tabular}

Table S3: Calculated rotational constants (GHz) for monomers, their complexes, and transition states (TS) at the MO6-2X/6-311++g(3df,3pd) level.

\begin{tabular}{|l|c|c|c|}
\hline & MO6-2X & MO6-2X & MO6-2X \\
\hline Species & A & B & C \\
\hline $\mathrm{HOCH}_{2} \mathrm{CH}_{2} \mathrm{CH}_{2} \mathrm{CHO}$ & 5.33 & 2.91 & 2.23 \\
\hline $\mathrm{H}_{2} \mathrm{O}$ & 829.88 & 433.02 & 284.55 \\
\hline $\mathrm{HONO}_{2}$ & 13.34 & 12.49 & 6.45 \\
\hline $\mathrm{HCOOH}$ & 78.27 & 12.24 & 10.59 \\
\hline $2-\mathrm{OH}-\mathrm{THF}_{2,3-\mathrm{DHF}}$ & 5.58 & 3.80 & 2.99 \\
\hline $\mathrm{HOCH}_{2} \mathrm{CH}_{2} \mathrm{CH}_{2} \mathrm{CHO}(\mathrm{TSa})$ & 8.17 & 7.9 & 4.26 \\
\hline $\mathrm{HOCH} \mathrm{CH}_{2} \mathrm{CH}_{2} \mathrm{CHO}(\mathrm{TSb})$ & 5.55 & 3.87 & 2.90 \\
\hline $\mathrm{HOCH}_{2} \mathrm{CH}_{2} \mathrm{CH}_{2} \mathrm{CHO}(\mathrm{PC})$ & 5.61 & 3.25 & 2.71 \\
\hline $\mathrm{HOCH}_{2} \mathrm{CH}_{2} \mathrm{CH}_{2} \mathrm{CHO} \bullet \mathrm{H}_{2} \mathrm{O}(\mathrm{RC} 1)$ & 4.22 & 2.61 & 2.59 \\
\hline $\mathrm{HOCH}_{2} \mathrm{CH}_{2} \mathrm{CH}_{2} \mathrm{CHO} \bullet \mathrm{H}_{2} \mathrm{O}(\mathrm{TS} 1)$ & 2.45 & 2.26 & 1.49 \\
\hline $\mathrm{HOCH}_{2} \mathrm{CH}_{2} \mathrm{CH}_{2} \mathrm{CHO} \bullet \mathrm{H}_{2} \mathrm{O}(\mathrm{PC} 1)$ & 3.76 & 2.27 & 1.74 \\
\hline $\mathrm{HOCH}_{2} \mathrm{CH}_{2} \mathrm{CH}_{2} \mathrm{CHO} \bullet \mathrm{H}_{2} \mathrm{O}(\mathrm{RC} 2)$ & 4.31 & 1.78 & 1.47 \\
\hline $\mathrm{HOCH}_{2} \mathrm{CH}_{2} \mathrm{CH}_{2} \mathrm{CHO} \bullet \mathrm{H}_{2} \mathrm{O}(\mathrm{TS} 2)$ & 2.97 & 2.19 & 1.80 \\
\hline
\end{tabular}




\begin{tabular}{|c|c|c|c|}
\hline $\mathrm{HOCH}_{2} \mathrm{CH}_{2} \mathrm{CH}_{2} \mathrm{CHO} \cdot \bullet \mathrm{H}_{2} \mathrm{O}(\mathrm{PC} 2)$ & 2.78 & 1.75 & 1.44 \\
\hline $\mathrm{HOCH}_{2} \mathrm{CH}_{2} \mathrm{CH}_{2} \mathrm{CHO} \bullet \bullet \mathrm{H}_{2} \mathrm{O}(\mathrm{RC} 1 \mathrm{~A})$ & 2.45 & 2.26 & 1.49 \\
\hline $\mathrm{HOCH}_{2} \mathrm{CH}_{2} \mathrm{CH}_{2} \mathrm{CHO} \bullet \cdot \mathrm{H}_{2} \mathrm{O}(\mathrm{TS} 1 \mathrm{~A})$ & 3.76 & 2.27 & 1.74 \\
\hline $\mathrm{HOCH}_{2} \mathrm{CH}_{2} \mathrm{CH}_{2} \mathrm{CHO} \bullet \cdot \mathrm{H}_{2} \mathrm{O}(\mathrm{PC} 1 \mathrm{~A})$ & 4.31 & 1.78 & 1.47 \\
\hline $\mathrm{HOCH}_{2} \mathrm{CH}_{2} \mathrm{CH}_{2} \mathrm{CHO} \bullet \cdot \mathrm{HO}_{2}(\mathrm{RC} 3)$ & 2.36 & 1.49 & 1.17 \\
\hline $\mathrm{HOCH}_{2} \mathrm{CH}_{2} \mathrm{CH}_{2} \mathrm{CHO} \bullet \cdot \mathrm{HO}_{2}(\mathrm{TS} 3)$ & 2.91 & 1.62 & 1.32 \\
\hline $\mathrm{HOCH}_{2} \mathrm{CH}_{2} \mathrm{CH}_{2} \mathrm{CHO} \bullet \cdot \mathrm{HO}_{2}(\mathrm{PC} 3)$ & 2.86 & 1.54 & 1.27 \\
\hline $2-\mathrm{OH}-\mathrm{THF} \bullet \cdot \mathrm{HO}_{2}(\mathrm{RC} 4)$ & 2.73 & 1.51 & 1.25 \\
\hline $2-\mathrm{OH}-\mathrm{THF} \bullet \bullet \mathrm{HO}_{2}(\mathrm{TS} 4)$ & 2.46 & 1.63 & 1.31 \\
\hline $2-\mathrm{OH}-\mathrm{THF} \bullet \bullet \mathrm{HO}_{2}(\mathrm{PC} 4)$ & 2.34 & 1.46 & 1.19 \\
\hline $\mathrm{HOCH}_{2} \mathrm{CH}_{2} \mathrm{CH}_{2} \mathrm{CHO} \bullet \bullet \mathrm{HO}_{2}(\mathrm{RC} 3 \mathrm{~A})$ & 2.64 & 1.32 & 1.01 \\
\hline $\mathrm{HOCH}_{2} \mathrm{CH}_{2} \mathrm{CH}_{2} \mathrm{CHO} \bullet \cdot \mathrm{HO}_{2}(\mathrm{TS} 3 \mathrm{~A})$ & 3.49 & 1.43 & 1.17 \\
\hline $\mathrm{HOCH}_{2} \mathrm{CH}_{2} \mathrm{CH}_{2} \mathrm{CHO} \bullet \bullet \mathrm{HO}_{2}(\mathrm{PC} 3 \mathrm{~A})$ & 3.76 & 1.27 & 1.06 \\
\hline $\mathrm{HOCH}_{2} \mathrm{CH}_{2} \mathrm{CH}_{2} \mathrm{CHO} \bullet$ FA (RC5) & 2.34 & 0.98 & 0.77 \\
\hline $\mathrm{HOCH}_{2} \mathrm{CH}_{2} \mathrm{CH}_{2} \mathrm{CHO} \bullet \cdot \mathrm{FA}$ (TS5) & 2.97 & 1.07 & 0.87 \\
\hline $\mathrm{HOCH}_{2} \mathrm{CH}_{2} \mathrm{CH}_{2} \mathrm{CHO} \bullet \cdot \mathrm{FA}(\mathrm{PC} 5)$ & 3.14 & 0.96 & 0.80 \\
\hline $\mathrm{HOCH}_{2} \mathrm{CH}_{2} \mathrm{CH}_{2} \mathrm{CHO} \bullet$ FA (RC5A) & 1.99 & 1.18 & 0.94 \\
\hline $\mathrm{HOCH}_{2} \mathrm{CH}_{2} \mathrm{CH}_{2} \mathrm{CHO} \bullet \cdot \mathrm{FA}(\mathrm{TS} 5 \mathrm{~A})$ & 2.23 & 1.36 & 1.16 \\
\hline $\mathrm{HOCH}_{2} \mathrm{CH}_{2} \mathrm{CH}_{2} \mathrm{CHO} \bullet \cdot \mathrm{FA}(\mathrm{PC} 5 \mathrm{~A})$ & 2.54 & 1.16 & 1.09 \\
\hline 2-OH-THF••FA (RC6) & 2.53 & 1.02 & 0.97 \\
\hline 2-OH-THF••FA (TS6) & 1.96 & 1.31 & 1.08 \\
\hline 2-OH-THF••FA (PC6) & 2.08 & 1.18 & 1.03 \\
\hline $\mathrm{HOCH}_{2} \mathrm{CH}_{2} \mathrm{CH}_{2} \mathrm{CHO} \bullet \bullet \mathrm{HNO}_{3}(\mathrm{RC} 7)$ & 2.37 & 0.68 & 0.57 \\
\hline $\mathrm{HOCH}_{2} \mathrm{CH}_{2} \mathrm{CH}_{2} \mathrm{CHO} \bullet \cdot \mathrm{HNO}_{3}(\mathrm{TS} 7)$ & 2.89 & 0.73 & 0.63 \\
\hline $\mathrm{HOCH}_{2} \mathrm{CH}_{2} \mathrm{CH}_{2} \mathrm{CHO} \bullet \cdot \mathrm{HNO}_{3}(\mathrm{PC} 7)$ & 2.54 & 0.81 & 0.77 \\
\hline
\end{tabular}




\begin{tabular}{|c|c|c|c|}
\hline $\mathrm{HOCH}_{2} \mathrm{CH}_{2} \mathrm{CH}_{2} \mathrm{CHO} \cdot \bullet \mathrm{HNO}_{3}(\mathrm{RC} 7 \mathrm{~A})$ & 2.37 & 0.68 & 0.57 \\
\hline $\mathrm{HOCH}_{2} \mathrm{CH}_{2} \mathrm{CH}_{2} \mathrm{CHO} \cdot \bullet \mathrm{HNO}_{3}(\mathrm{TS} 7 \mathrm{~A})$ & 2.06 & 0.95 & 0.86 \\
\hline $\mathrm{HOCH}_{2} \mathrm{CH}_{2} \mathrm{CH}_{2} \mathrm{CHO} \cdot \bullet \mathrm{HNO}_{3}(\mathrm{PC} 7 \mathrm{~A})$ & 3.11 & 0.68 & 0.60 \\
\hline 2-OH-THF••-HNO 3 (RC8) & 2.43 & 0.76 & 0.67 \\
\hline 2-OH-THF••HNO 3 (TS8) & 2.03 & 0.78 & 0.65 \\
\hline 2-OH-THF••-HNO 3 (PC8) & 1.76 & 0.91 & 0.74 \\
\hline trans $-\mathrm{H}_{2} \mathrm{SO}_{4}$ & 5.18 & 5.06 & 4.89 \\
\hline $\mathrm{HOCH}_{2} \mathrm{CH}_{2} \mathrm{CH}_{2} \mathrm{CHO} \bullet \bullet$ trans- $\mathrm{H}_{2} \mathrm{SO}_{4}(\mathrm{RC} 9)$ & 1.54 & 0.65 & 0.58 \\
\hline $\mathrm{HOCH}_{2} \mathrm{CH}_{2} \mathrm{CH}_{2} \mathrm{CHO} \bullet \bullet$ trans- $\mathrm{H}_{2} \mathrm{SO}_{4}$ (TS9) & 1.65 & 0.69 & 0.63 \\
\hline $\mathrm{HOCH}_{2} \mathrm{CH}_{2} \mathrm{CH}_{2} \mathrm{CHO} \bullet$ trans- $\mathrm{H}_{2} \mathrm{SO}_{4}(\mathrm{PC} 9)$ & 1.72 & 0.68 & 0.62 \\
\hline $2-\mathrm{OH}-\mathrm{THF} \bullet \bullet$ trans- $\mathrm{H}_{2} \mathrm{SO}_{4}(\mathrm{RC} 10)$ & 2.10 & 0.52 & 0.48 \\
\hline 2-OH-THF••trans- $\mathrm{H}_{2} \mathrm{SO}_{4}(\mathrm{TS} 10)$ & 1.29 & 0.76 & 0.60 \\
\hline 2-OH-THF••trans- $\mathrm{H}_{2} \mathrm{SO}_{4}(\mathrm{PC} 10)$ & 1.43 & 0.68 & 0.57 \\
\hline $\mathrm{HOCH}_{2} \mathrm{CH}_{2} \mathrm{CH}_{2} \mathrm{CHO} \bullet$ trans- $\mathrm{H}_{2} \mathrm{SO}_{4}(\mathrm{RC} 9 \mathrm{~A})$ & 1.85 & 0.54 & 0.48 \\
\hline $\mathrm{HOCH}_{2} \mathrm{CH}_{2} \mathrm{CH}_{2} \mathrm{CHO} \bullet$ trans- $\mathrm{H}_{2} \mathrm{SO}_{4}(\mathrm{TS} 9 \mathrm{~A})$ & 2.13 & 0.53 & 0.49 \\
\hline $\mathrm{HOCH}_{2} \mathrm{CH}_{2} \mathrm{CH}_{2} \mathrm{CHO} \bullet \bullet$ trans $-\mathrm{H}_{2} \mathrm{SO}_{4}(\mathrm{PC} 9 \mathrm{~A})$ & 2.30 & 0.50 & 0.47 \\
\hline cis- $\mathrm{H}_{2} \mathrm{SO}_{4}$ & 5.17 & 5.10 & 4.85 \\
\hline $\mathrm{HOCH}_{2} \mathrm{CH}_{2} \mathrm{CH}_{2} \mathrm{CHO} \bullet$ cis- $\mathrm{H}_{2} \mathrm{SO}_{4}(\mathrm{RC} 9 \mathrm{~B})$ & 1.54 & 0.65 & 0.59 \\
\hline $\mathrm{HOCH}_{2} \mathrm{CH}_{2} \mathrm{CH}_{2} \mathrm{CHO} \cdot \bullet$ cis- $\mathrm{H}_{2} \mathrm{SO}_{4}$ (TS9B) & 1.65 & 0.70 & 0.63 \\
\hline $\mathrm{HOCH}_{2} \mathrm{CH}_{2} \mathrm{CH}_{2} \mathrm{CHO} \cdot \bullet$ cis- $\mathrm{H}_{2} \mathrm{SO}_{4}(\mathrm{PC} 9 \mathrm{~B})$ & 1.72 & 0.69 & 0.62 \\
\hline$\cdot \mathrm{OH}$ & 565.60 & & \\
\hline 2-OH-THF••OH (TS11) & 3.83 & 1.86 & 1.65 \\
\hline 2-OH-THF••OH (RC11) & 4.23 & 1.85 & 1.50 \\
\hline 2-OH-THF••OH (PC11) & 4.60 & 1.69 & 1.30 \\
\hline 2-OH-THF••OH (P11) & 6.92 & 3.47 & 2.52 \\
\hline
\end{tabular}




\begin{tabular}{|c|c|c|c|}
\hline 2-OH-THF••OH (TS12) & 3.81 & 2.10 & 1.90 \\
\hline 2-OH-THF••OH (RC12) & 3.22 & 2.24 & 1.69 \\
\hline 2-OH-THF••OH (PC12) & 3.07 & 2.25 & 1.79 \\
\hline 2-OH-THF••OH (P12) & 6.14 & 3.81 & 2.86 \\
\hline 2-OH-THF••OH (TS13) & 3.45 & 2.12 & 1.92 \\
\hline 2-OH-THF••OH (RC13) & 3.28 & 2.00 & 1.76 \\
\hline 2-OH-THF••OH (PC13) & 3.61 & 1.84 & 1.66 \\
\hline 2-OH-THF••OH (P13) & 6.30 & 3.67 & 2.86 \\
\hline 2-OH-THF••OH (TS14) & 3.16 & 2.43 & 1.78 \\
\hline 2-OH-THF••OH (RC14) & 2.92 & 2.36 & 1.92 \\
\hline 2-OH-THF••OH (PC14) & 2.94 & 2.30 & 1.81 \\
\hline 2-OH-THF••OH (TS15) & 5.07 & 1.48 & 1.34 \\
\hline 2-OH-THF••OH (RC15) & 4.64 & 1.49 & 1.36 \\
\hline 2-OH-THF••OH (PC15) & 3.61 & 1.84 & 1.75 \\
\hline 2-OH-THF••OH (P14) & 5.73 & 3.96 & 3.04 \\
\hline 2-OH-THF••OH (TS16) & 3.37 & 1.92 & 1.67 \\
\hline 2-OH-THF••OH (PC16) & 3.03 & 2.10 & 1.80 \\
\hline 2-OH-THF••OH (TS17) & 4.31 & 1.75 & 1.62 \\
\hline 2-OH-THF••OH (PC17) & 3.16 & 1.98 & 1.70 \\
\hline 2-OH-THF••OH (P15) & 5.77 & 3.95 & 3.06 \\
\hline 2-OH-THF••OH (TS18) & 2.77 & 2.65 & 2.01 \\
\hline 2-OH-THF••OH (PC18) & 3.02 & 2.11 & 1.79 \\
\hline $\mathrm{H}_{2} \mathrm{O}$ & 830.74 & 433.04 & 284.66 \\
\hline${ }^{3} \mathrm{O}_{2}$ & 44.80 & & \\
\hline $2-\mathrm{OH}-\mathrm{THF}^{\bullet} \bullet \mathrm{O}_{2}\left(\mathrm{RO}_{2}\right)$ & 3.74 & 1.66 & 1.48 \\
\hline
\end{tabular}




\begin{tabular}{|l|c|c|c|}
\hline 2-OH-THF $\bullet \mathrm{O}_{2}$ (TS19) & 2.79 & 2.04 & 1.66 \\
\hline 2-OH-THF $\bullet \mathrm{O}_{2}(\mathrm{PC} 19)$ & 2.37 & 1.51 & 1.17 \\
\hline Succinaldehyde & 5.77 & 2.89 & 2.48 \\
\hline $\mathrm{HO}_{2}$ & 630.48 & 34.93 & 33.09 \\
\hline 2-OH-THF $\bullet \mathrm{O}_{2}(\mathrm{TS} 20)$ & 3.01 & 1.88 & 1.72 \\
\hline 2-OH-THF $\bullet \mathrm{O}_{2}(\mathrm{PC} 20)$ & 3.37 & 1.42 & 1.25 \\
\hline 2,3-DH-2-FOH & 6.51 & 3.96 & 2.96 \\
\hline
\end{tabular}

Table S4: Calculated positive frequencies $\left(\mathrm{cm}^{-1}\right)$ for monomers, their complexes, and transition states (TS) at the MO6-2X level.

\begin{tabular}{|c|c|c|c|c|c|}
\hline \multirow{2}{*}{$\begin{array}{l}\text { Species } \\
\mathrm{HOCH}_{2} \mathrm{CH}_{2} \mathrm{CH}_{2} \mathrm{CHO}\end{array}$} & \multicolumn{5}{|c|}{ Frequencies } \\
\hline & $\begin{array}{l}114 \\
458 \\
888 \\
1152 \\
1381 \\
1487 \\
3036 \\
3863\end{array}$ & $\begin{array}{l}144 \\
508 \\
956 \\
1194 \\
1399 \\
1524 \\
3058\end{array}$ & $\begin{array}{l}160 \\
666 \\
1006 \\
1249 \\
1421 \\
1855 \\
3072\end{array}$ & $\begin{array}{l}261 \\
701 \\
1043 \\
1294 \\
1434 \\
2986 \\
3080\end{array}$ & $\begin{array}{l}300 \\
844 \\
1120 \\
1362 \\
1446 \\
3032 \\
3110\end{array}$ \\
\hline $\mathrm{H}_{2} \mathrm{O}$ & 1620 & 3891 & 3993 & & \\
\hline \multicolumn{6}{|l|}{$\mathrm{HO}_{2}$} \\
\hline HCOOH (FA) & $\begin{array}{l}648 \\
1413\end{array}$ & $\begin{array}{l}687 \\
1880\end{array}$ & $\begin{array}{l}1067 \\
3133\end{array}$ & $\begin{array}{l}1162 \\
3800\end{array}$ & 1319 \\
\hline $\mathrm{HOCH}_{2} \mathrm{CH}_{2} \mathrm{CH}_{2} \mathrm{CHO}(\mathrm{TSa})$ & $\begin{array}{l}-1517 \\
577 \\
924 \\
1145 \\
1324 \\
1488 \\
3075 \\
3151\end{array}$ & $\begin{array}{c}200 \\
638 \\
950 \\
1175 \\
1345 \\
1503 \\
3083\end{array}$ & $\begin{array}{c}260 \\
764 \\
1006 \\
1237 \\
1356 \\
1524 \\
3088\end{array}$ & $\begin{array}{c}408 \\
871 \\
1054 \\
1263 \\
1394 \\
2063 \\
3130\end{array}$ & $\begin{array}{l}524 \\
888 \\
1073 \\
1314 \\
1479 \\
3068 \\
3143\end{array}$ \\
\hline 2-OH-THF & 84 & 206 & 355 & 422 & 520 \\
\hline
\end{tabular}




\begin{tabular}{|c|c|c|c|c|c|}
\hline & $\begin{array}{l}639 \\
956 \\
1163 \\
1328 \\
1488 \\
3095 \\
3904\end{array}$ & $\begin{array}{l}790 \\
966 \\
1202 \\
1348 \\
1507 \\
3096\end{array}$ & $\begin{array}{l}872 \\
1017 \\
1214 \\
1372 \\
1538 \\
3107\end{array}$ & $\begin{array}{l}879 \\
1055 \\
1257 \\
1408 \\
3057 \\
3150\end{array}$ & $\begin{array}{l}942 \\
1130 \\
1266 \\
1445 \\
3081 \\
3160\end{array}$ \\
\hline 2-OH-THF (TSb) & $\begin{array}{l}-1800 \\
474 \\
868 \\
1039 \\
1255 \\
1486 \\
3075 \\
3862\end{array}$ & $\begin{array}{l}159 \\
600 \\
876 \\
1074 \\
1314 \\
1511 \\
3116\end{array}$ & $\begin{array}{l}174 \\
607 \\
956 \\
1096 \\
1319 \\
1523 \\
3160\end{array}$ & $\begin{array}{l}238 \\
703 \\
976 \\
1209 \\
1385 \\
1674 \\
3215\end{array}$ & $\begin{array}{l}420 \\
787 \\
1001 \\
1243 \\
1422 \\
3045 \\
3271\end{array}$ \\
\hline 2-OH-THF (PC) & $\begin{array}{l}32 \\
285 \\
844 \\
1085 \\
1311 \\
1634 \\
3150 \\
3970\end{array}$ & $\begin{array}{l}115 \\
463 \\
851 \\
1109 \\
1329 \\
1693 \\
3233\end{array}$ & $\begin{array}{l}129 \\
476 \\
941 \\
1090 \\
1424 \\
3056 \\
3160\end{array}$ & $\begin{array}{l}148 \\
715 \\
960 \\
1225 \\
1502 \\
3095 \\
3264\end{array}$ & $\begin{array}{l}207 \\
754 \\
1032 \\
1269 \\
1527 \\
3102 \\
3833\end{array}$ \\
\hline 2,3-DHF & $\begin{array}{l}134 \\
858 \\
1074 \\
1307 \\
1695 \\
3230\end{array}$ & $\begin{array}{l}460 \\
944 \\
1107 \\
1334 \\
3038 \\
3257\end{array}$ & $\begin{array}{l}679 \\
948 \\
1175 \\
1416 \\
3058\end{array}$ & $\begin{array}{l}733 \\
973 \\
1220 \\
1495 \\
3096\end{array}$ & $\begin{array}{l}843 \\
1030 \\
1257 \\
1523 \\
3139\end{array}$ \\
\hline $\mathrm{HOCH}_{2} \mathrm{CH}_{2} \mathrm{CH}_{2} \mathrm{CHO} \cdot \mathrm{H}_{2} \mathrm{O}(\mathrm{RC} 1)$ & $\begin{array}{l}76 \\
215 \\
497 \\
853 \\
1115 \\
1362 \\
1453 \\
3001 \\
3090\end{array}$ & $\begin{array}{l}102 \\
258 \\
535 \\
872 \\
1146 \\
1399 \\
1492 \\
3031 \\
3117\end{array}$ & $\begin{array}{l}115 \\
276 \\
643 \\
961 \\
1195 \\
1411 \\
1514 \\
3040 \\
3724\end{array}$ & $\begin{array}{l}131 \\
316 \\
736 \\
1025 \\
1247 \\
1429 \\
1631 \\
3066 \\
3778\end{array}$ & $\begin{array}{l}199 \\
360 \\
783 \\
1043 \\
1298 \\
1447 \\
1851 \\
3078 \\
3962\end{array}$ \\
\hline $\mathrm{HOCH}_{2} \mathrm{CH}_{2} \mathrm{CH}_{2} \mathrm{CHO} \cdot \mathrm{H}_{2} \mathrm{O}(\mathrm{TS} 1)$ & $\begin{array}{l}-1157 \\
389 \\
648 \\
931\end{array}$ & $\begin{array}{l}65 \\
502 \\
736 \\
962\end{array}$ & $\begin{array}{l}141 \\
547 \\
783 \\
1015\end{array}$ & $\begin{array}{l}232 \\
580 \\
884 \\
1064\end{array}$ & $\begin{array}{l}374 \\
613 \\
894 \\
1095\end{array}$ \\
\hline
\end{tabular}




\begin{tabular}{|c|c|c|c|c|c|}
\hline & $\begin{array}{l}1180 \\
1335 \\
1487 \\
1718 \\
3087\end{array}$ & $\begin{array}{l}1216 \\
1349 \\
1492 \\
1914 \\
3122\end{array}$ & $\begin{array}{l}1258 \\
1360 \\
1501 \\
3045 \\
3140\end{array}$ & $\begin{array}{l}1283 \\
1397 \\
1537 \\
3068 \\
3148\end{array}$ & $\begin{array}{l}1304 \\
1479 \\
1576 \\
3069 \\
3944\end{array}$ \\
\hline $\mathrm{HOCH}_{2} \mathrm{CH}_{2} \mathrm{CH}_{2} \mathrm{CHO} \cdot \bullet \mathrm{H}_{2} \mathrm{O}(\mathrm{PC} 1)$ & $\begin{array}{l}55 \\
226 \\
639 \\
941 \\
1110 \\
1292 \\
1465 \\
3069 \\
3142\end{array}$ & $\begin{array}{l}73 \\
339 \\
691 \\
944 \\
1165 \\
1329 \\
1486 \\
3077 \\
3153\end{array}$ & $\begin{array}{l}151 \\
429 \\
791 \\
964 \\
1214 \\
1354 \\
1503 \\
3091 \\
3724\end{array}$ & $\begin{array}{l}181 \\
513 \\
866 \\
1021 \\
1218 \\
1378 \\
1538 \\
3098 \\
3790\end{array}$ & $\begin{array}{l}206 \\
625 \\
883 \\
1064 \\
1268 \\
1409 \\
1625 \\
3116 \\
3969\end{array}$ \\
\hline $\mathrm{HOCH}_{2} \mathrm{CH}_{2} \mathrm{CH}_{2} \mathrm{CHO} \cdot \bullet \mathrm{H}_{2} \mathrm{O}(\mathrm{RC} 1 \mathrm{~A})$ & \begin{tabular}{|l}
76 \\
216 \\
497 \\
853 \\
1115 \\
1362 \\
1453 \\
3001 \\
3090
\end{tabular} & $\begin{array}{l}101 \\
256 \\
536 \\
872 \\
1146 \\
1399 \\
1492 \\
3031 \\
3117\end{array}$ & $\begin{array}{l}115 \\
276 \\
642 \\
961 \\
1196 \\
1412 \\
1514 \\
3040 \\
3723\end{array}$ & $\begin{array}{l}131 \\
317 \\
736 \\
1025 \\
1247 \\
1429 \\
1631 \\
3066 \\
3777\end{array}$ & $\begin{array}{l}200 \\
357 \\
785 \\
1043 \\
1298 \\
1447 \\
1851 \\
3079 \\
3962\end{array}$ \\
\hline $\mathrm{HOCH}_{2} \mathrm{CH}_{2} \mathrm{CH}_{2} \mathrm{CHO} \bullet \cdot \mathrm{H}_{2} \mathrm{O}(\mathrm{TS} 1 \mathrm{~A})$ & \begin{tabular}{|l}
-1166 \\
391 \\
639 \\
931 \\
1181 \\
1334 \\
1488 \\
1707 \\
3093
\end{tabular} & $\begin{array}{l}71 \\
522 \\
735 \\
963 \\
1217 \\
1348 \\
1490 \\
1922 \\
3128\end{array}$ & $\begin{array}{l}147 \\
553 \\
785 \\
1015 \\
1256 \\
1359 \\
1501 \\
3054 \\
3142\end{array}$ & $\begin{array}{l}234 \\
570 \\
886 \\
1063 \\
1282 \\
1397 \\
1539 \\
3069 \\
3153\end{array}$ & $\begin{array}{l}373 \\
614 \\
896 \\
1095 \\
1303 \\
1481 \\
1574 \\
3078 \\
3940\end{array}$ \\
\hline $\mathrm{HOCH}_{2} \mathrm{CH}_{2} \mathrm{CH}_{2} \mathrm{CHO} \cdot \bullet \mathrm{H}_{2} \mathrm{O}(\mathrm{PC} 1 \mathrm{~A})$ & \begin{tabular}{|l}
55 \\
232 \\
639 \\
941 \\
1110 \\
1292 \\
1465 \\
3070 \\
3142
\end{tabular} & $\begin{array}{l}74 \\
339 \\
692 \\
944 \\
1165 \\
1329 \\
1486 \\
3077 \\
3153\end{array}$ & $\begin{array}{l}151 \\
429 \\
791 \\
964 \\
1214 \\
1355 \\
1503 \\
3090 \\
3725\end{array}$ & $\begin{array}{l}181 \\
513 \\
866 \\
1021 \\
1218 \\
1378 \\
1538 \\
3098 \\
3790\end{array}$ & $\begin{array}{l}207 \\
624 \\
883 \\
1064 \\
1268 \\
1409 \\
1626 \\
3116 \\
3969\end{array}$ \\
\hline $\mathrm{HOCH}_{2} \mathrm{CH}_{2} \mathrm{CH}_{2} \mathrm{CHO} \cdot \mathrm{H}_{2} \mathrm{O}(\mathrm{RC} 2)$ & 79 & 112 & 142 & 164 & 188 \\
\hline
\end{tabular}




\begin{tabular}{|c|c|c|c|c|c|}
\hline & $\begin{array}{l}243 \\
630 \\
932 \\
1122 \\
1273 \\
1436 \\
3073 \\
3147\end{array}$ & $\begin{array}{l}373 \\
660 \\
955 \\
1163 \\
1309 \\
1491 \\
3079 \\
3162\end{array}$ & $\begin{array}{l}376 \\
801 \\
995 \\
1223 \\
1331 \\
1513 \\
3106 \\
3747\end{array}$ & $\begin{array}{l}431 \\
814 \\
1029 \\
1243 \\
1372 \\
1545 \\
3125 \\
3881\end{array}$ & $\begin{array}{l}542 \\
877 \\
1061 \\
1264 \\
1407 \\
1647 \\
3137 \\
3964\end{array}$ \\
\hline $\mathrm{HOCH}_{2} \mathrm{CH}_{2} \mathrm{CH}_{2} \mathrm{CHO} \cdot \bullet \mathrm{H}_{2} \mathrm{O}(\mathrm{TS} 2)$ & $\begin{array}{l}-1469 \\
306 \\
609 \\
878 \\
1054 \\
1248 \\
1455 \\
1660 \\
3158\end{array}$ & $\begin{array}{l}72 \\
423 \\
643 \\
896 \\
1090 \\
1300 \\
1482 \\
1861 \\
3179\end{array}$ & $\begin{array}{l}142 \\
494 \\
689 \\
956 \\
1114 \\
1317 \\
1507 \\
3051 \\
3257\end{array}$ & $\begin{array}{l}196 \\
531 \\
715 \\
976 \\
1213 \\
1348 \\
1521 \\
3074 \\
3910\end{array}$ & $\begin{array}{l}248 \\
541 \\
798 \\
1007 \\
1242 \\
1400 \\
1587 \\
3119 \\
3925\end{array}$ \\
\hline $\mathrm{HOCH}_{2} \mathrm{CH}_{2} \mathrm{CH}_{2} \mathrm{CHO} \cdot \bullet \mathrm{H}_{2} \mathrm{O}(\mathrm{PC} 2)$ & $\begin{array}{l}34 \\
190 \\
450 \\
765 \\
966 \\
1227 \\
1499 \\
3060 \\
3268\end{array}$ & $\begin{array}{l}80 \\
202 \\
461 \\
844 \\
1027 \\
1264 \\
1535 \\
3100 \\
3794\end{array}$ & $\begin{array}{l}113 \\
246 \\
478 \\
855 \\
1082 \\
1312 \\
1619 \\
3108 \\
3834\end{array}$ & $\begin{array}{l}137 \\
288 \\
583 \\
946 \\
1112 \\
1340 \\
1669 \\
3158 \\
3917\end{array}$ & $\begin{array}{l}146 \\
350 \\
697 \\
961 \\
1182 \\
1426 \\
1689 \\
3241 \\
3955\end{array}$ \\
\hline $\mathrm{HOCH}_{2} \mathrm{CH}_{2} \mathrm{CH}_{2} \mathrm{CHO} \bullet \cdot \mathrm{HO}_{2}(\mathrm{RC} 3)$ & $\begin{array}{l}72 \\
192 \\
469 \\
859 \\
1117 \\
1321 \\
1436 \\
1815 \\
3096\end{array}$ & $\begin{array}{l}94 \\
216 \\
558 \\
906 \\
1138 \\
1347 \\
1452 \\
3036 \\
3102\end{array}$ & $\begin{array}{l}130 \\
293 \\
588 \\
951 \\
1211 \\
1382 \\
1484 \\
3045 \\
3110\end{array}$ & $\begin{array}{l}138 \\
312 \\
769 \\
1026 \\
1238 \\
1398 \\
1516 \\
3047 \\
3129\end{array}$ & $\begin{array}{l}152 \\
360 \\
821 \\
1082 \\
1294 \\
1426 \\
1611 \\
3050 \\
3804\end{array}$ \\
\hline $\mathrm{HOCH}_{2} \mathrm{CH}_{2} \mathrm{CH}_{2} \mathrm{CHO} \cdot \bullet \mathrm{HO}_{2}(\mathrm{TS} 3)$ & $\begin{array}{l}-700 \\
250 \\
596 \\
929 \\
1166 \\
1322 \\
1400\end{array}$ & $\begin{array}{l}63 \\
361 \\
623 \\
942 \\
1197 \\
1358 \\
1473\end{array}$ & $\begin{array}{l}94 \\
447 \\
710 \\
1022 \\
1236 \\
1363 \\
1503\end{array}$ & $\begin{array}{l}175 \\
512 \\
858 \\
1082 \\
1282 \\
1373 \\
1524\end{array}$ & $\begin{array}{l}239 \\
569 \\
905 \\
1149 \\
1292 \\
1391 \\
1572\end{array}$ \\
\hline
\end{tabular}




\begin{tabular}{|c|c|c|c|c|c|}
\hline & $\begin{array}{l}1634 \\
3079\end{array}$ & $\begin{array}{l}1748 \\
3112\end{array}$ & $\begin{array}{l}2387 \\
3127\end{array}$ & $\begin{array}{l}3062 \\
3131\end{array}$ & $\begin{array}{l}3074 \\
3144\end{array}$ \\
\hline \multirow[t]{9}{*}{$\mathrm{HOCH}_{2} \mathrm{CH}_{2} \mathrm{CH}_{2} \mathrm{CHO} \cdot \bullet \mathrm{HO}_{2}(\mathrm{PC} 3)$} & 49 & 65 & 120 & 169 & 197 \\
\hline & 246 & 288 & 415 & 543 & 564 \\
\hline & 630 & 658 & 762 & 864 & 876 \\
\hline & 915 & 958 & 970 & 1052 & 1098 \\
\hline & 1123 & 1188 & 1212 & 1221 & 1276 \\
\hline & 1314 & 1321 & 1337 & 1365 & 1389 \\
\hline & 1412 & 1471 & 1483 & 1500 & 1539 \\
\hline & 1612 & 3055 & 3061 & 3069 & 3113 \\
\hline & 3120 & 3130 & 3138 & 3143 & 3762 \\
\hline \multirow[t]{9}{*}{$2-\mathrm{OH}-\mathrm{THF} \bullet \cdot \mathrm{HO}_{2}(\mathrm{RC} 4)$} & 68 & 105 & 110 & 143 & 161 \\
\hline & 238 & 244 & 369 & 448 & 552 \\
\hline & 626 & 686 & 775 & 839 & 870 \\
\hline & 932 & 954 & 993 & 1028 & 1065 \\
\hline & 1118 & 1168 & 1220 & 1239 & 1259 \\
\hline & 1276 & 1303 & 1321 & 1333 & 1377 \\
\hline & 1412 & 1436 & 1488 & 1510 & 1531 \\
\hline & 1591 & 3077 & 3085 & 3108 & 3117 \\
\hline & 3141 & 3151 & 3164 & 3313 & 3882 \\
\hline \multirow{9}{*}{$2-\mathrm{OH}-\mathrm{THF} \bullet \cdot \mathrm{HO}_{2}(\mathrm{TS} 4)$} & -262 & 61 & 68 & 146 & 158 \\
\hline & 186 & 255 & 296 & 322 & 375 \\
\hline & 521 & 625 & 675 & 789 & 831 \\
\hline & 864 & 936 & 950 & 968 & 996 \\
\hline & 1007 & 1061 & 1110 & 1193 & 1225 \\
\hline & 1245 & 1284 & 1320 & 1327 & 1344 \\
\hline & 1390 & 1480 & 1489 & 1508 & 1551 \\
\hline & 1708 & 2080 & 2702 & 3076 & 3100 \\
\hline & 3155 & 3162 & 3182 & 3293 & 3933 \\
\hline \multirow[t]{9}{*}{$2-\mathrm{OH}-\mathrm{THF} \bullet \bullet \mathrm{HO}_{2}(\mathrm{PC} 4)$} & 39 & 47 & 70 & 82 & 136 \\
\hline & 144 & 183 & 216 & 293 & 331 \\
\hline & 410 & 469 & 639 & 687 & 754 \\
\hline & 843 & 857 & 892 & 943 & 947 \\
\hline & 962 & 1028 & 1082 & 1099 & 1167 \\
\hline & 1227 & 1266 & 1305 & 1315 & 1338 \\
\hline & 1416 & 1500 & 1520 & 1592 & 1638 \\
\hline & 1705 & 3074 & 3084 & 3122 & 3153 \\
\hline & 3211 & 3241 & 3268 & 3629 & 3936 \\
\hline \multirow[t]{4}{*}{$\mathrm{HOCH}_{2} \mathrm{CH}_{2} \mathrm{CH}_{2} \mathrm{CHO} \cdot \bullet \mathrm{HO}_{2}(\mathrm{RC} 3 \mathrm{~A})$} & 34 & 88 & 104 & 121 & 144 \\
\hline & 187 & 226 & 242 & 307 & 319 \\
\hline & 500 & 597 & 675 & 692 & 828 \\
\hline & 861 & 879 & 957 & 1018 & 1049 \\
\hline
\end{tabular}




\begin{tabular}{|c|c|c|c|c|c|}
\hline & $\begin{array}{l}1107 \\
1322 \\
1430 \\
1810 \\
3094\end{array}$ & $\begin{array}{l}1135 \\
1349 \\
1472 \\
3050 \\
3113\end{array}$ & $\begin{array}{l}1178 \\
1375 \\
1489 \\
3060 \\
3123\end{array}$ & $\begin{array}{l}1228 \\
1401 \\
1515 \\
3063 \\
3320\end{array}$ & $\begin{array}{l}1285 \\
1425 \\
1567 \\
3069 \\
3737\end{array}$ \\
\hline $\mathrm{HOCH}_{2} \mathrm{CH}_{2} \mathrm{CH}_{2} \mathrm{CHO} \cdot \bullet \mathrm{HO}_{2}(\mathrm{TS} 3 \mathrm{~A})$ & $\begin{array}{l}-600 \\
235 \\
656 \\
925 \\
1162 \\
1304 \\
1399 \\
1605 \\
3083\end{array}$ & $\begin{array}{l}75 \\
329 \\
703 \\
960 \\
1189 \\
1340 \\
1480 \\
1743 \\
3087\end{array}$ & $\begin{array}{l}96 \\
384 \\
844 \\
1023 \\
1233 \\
1357 \\
1491 \\
2433 \\
3127\end{array}$ & $\begin{array}{l}164 \\
520 \\
878 \\
1067 \\
1260 \\
1367 \\
1523 \\
3074 \\
3138\end{array}$ & $\begin{array}{l}208 \\
588 \\
897 \\
1097 \\
1280 \\
1380 \\
1551 \\
3078 \\
3147\end{array}$ \\
\hline $\mathrm{HOCH}_{2} \mathrm{CH}_{2} \mathrm{CH}_{2} \mathrm{CHO} \cdot \bullet \mathrm{HO}_{2}(\mathrm{PC} 3 \mathrm{~A})$ & $\begin{array}{l}54 \\
218 \\
650 \\
931 \\
1095 \\
1298 \\
1406 \\
1651 \\
3107\end{array}$ & $\begin{array}{l}67 \\
285 \\
794 \\
941 \\
1169 \\
1324 \\
1468 \\
3068 \\
3120\end{array}$ & $\begin{array}{l}102 \\
434 \\
809 \\
963 \\
1217 \\
1333 \\
1489 \\
3085 \\
3155\end{array}$ & $\begin{array}{l}124 \\
513 \\
863 \\
1030 \\
1225 \\
1357 \\
1510 \\
3092 \\
3164\end{array}$ & $\begin{array}{l}182 \\
641 \\
880 \\
1071 \\
1269 \\
1378 \\
1533 \\
3101 \\
3743\end{array}$ \\
\hline $\mathrm{HOCH}_{2} \mathrm{CH}_{2} \mathrm{CH}_{2} \mathrm{CHO} \bullet \cdot \mathrm{FA}(\mathrm{RC} 5)$ & $\begin{array}{l}39 \\
177 \\
331 \\
837 \\
1021 \\
1183 \\
1375 \\
1447 \\
1833 \\
3089 \\
3717\end{array}$ & $\begin{array}{l}52 \\
195 \\
502 \\
861 \\
1052 \\
1229 \\
1404 \\
1473 \\
3044 \\
3114\end{array}$ & $\begin{array}{l}86 \\
211 \\
599 \\
879 \\
1095 \\
1267 \\
1407 \\
1488 \\
3053 \\
3116\end{array}$ & $\begin{array}{l}112 \\
243 \\
681 \\
934 \\
1111 \\
1287 \\
1432 \\
1513 \\
3063 \\
3121\end{array}$ & $\begin{array}{l}133 \\
306 \\
697 \\
961 \\
1137 \\
1348 \\
1436 \\
1805 \\
3066 \\
3313\end{array}$ \\
\hline $\mathrm{HOCH}_{2} \mathrm{CH}_{2} \mathrm{CH}_{2} \mathrm{CHO} \bullet \mathrm{FA}$ (TS5) & $\begin{array}{l}-642 \\
214 \\
580 \\
878 \\
1067 \\
1218 \\
1365 \\
1480\end{array}$ & $\begin{array}{l}60 \\
252 \\
620 \\
894 \\
1098 \\
1245 \\
1391 \\
1493\end{array}$ & $\begin{array}{l}77 \\
299 \\
647 \\
920 \\
1100 \\
1284 \\
1402 \\
1521\end{array}$ & $\begin{array}{l}138 \\
356 \\
722 \\
960 \\
1149 \\
1338 \\
1443 \\
1532\end{array}$ & $\begin{array}{l}170 \\
494 \\
798 \\
1020 \\
1186 \\
1357 \\
1453 \\
1558\end{array}$ \\
\hline
\end{tabular}




\begin{tabular}{|c|c|c|c|c|c|}
\hline & $\begin{array}{l}1620 \\
3082 \\
3145\end{array}$ & $\begin{array}{l}1772 \\
3092\end{array}$ & $\begin{array}{l}2507 \\
3100\end{array}$ & $\begin{array}{l}3073 \\
3124\end{array}$ & $\begin{array}{l}3077 \\
3135\end{array}$ \\
\hline $\mathrm{HOCH}_{2} \mathrm{CH}_{2} \mathrm{CH}_{2} \mathrm{CHO} \bullet \cdot \mathrm{FA}(\mathrm{PC} 5)$ & $\begin{array}{l}41 \\
198 \\
644 \\
882 \\
1029 \\
1218 \\
1332 \\
1465 \\
1818 \\
3109 \\
3727\end{array}$ & $\begin{array}{l}50 \\
217 \\
706 \\
929 \\
1072 \\
1224 \\
1357 \\
1485 \\
3069 \\
3119\end{array}$ & $\begin{array}{l}90 \\
237 \\
726 \\
941 \\
1095 \\
1270 \\
1378 \\
1496 \\
3090 \\
3149\end{array}$ & $\begin{array}{l}109 \\
440 \\
802 \\
961 \\
1105 \\
1286 \\
1405 \\
1512 \\
3101 \\
3158\end{array}$ & $\begin{array}{l}163 \\
513 \\
866 \\
1008 \\
1171 \\
1306 \\
1425 \\
1537 \\
3103 \\
3167\end{array}$ \\
\hline $\mathrm{HOCH}_{2} \mathrm{CH}_{2} \mathrm{CH}_{2} \mathrm{CHO} \bullet \mathrm{FA}(\mathrm{RC} 5 \mathrm{~A})$ & $\begin{array}{l}66 \\
163 \\
312 \\
819 \\
1020 \\
1177 \\
1381 \\
1445 \\
1837 \\
3094 \\
3764\end{array}$ & $\begin{array}{l}70 \\
198 \\
496 \\
859 \\
1047 \\
1233 \\
1402 \\
1473 \\
3046 \\
3109\end{array}$ & $\begin{array}{l}87 \\
214 \\
599 \\
869 \\
1091 \\
1265 \\
1410 \\
1489 \\
3054 \\
3122\end{array}$ & $\begin{array}{l}120 \\
236 \\
650 \\
952 \\
1107 \\
1289 \\
1438 \\
1509 \\
3066 \\
3123\end{array}$ & $\begin{array}{l}152 \\
296 \\
701 \\
967 \\
1136 \\
1357 \\
1442 \\
1810 \\
3074 \\
3305\end{array}$ \\
\hline $\mathrm{HOCH}_{2} \mathrm{CH}_{2} \mathrm{CH}_{2} \mathrm{CHO} \bullet \mathrm{FA}(\mathrm{TS} 5 \mathrm{~A})$ & $\begin{array}{l}-724 \\
243 \\
511 \\
851 \\
1072 \\
1244 \\
1386 \\
1484 \\
1621 \\
3081 \\
3148\end{array}$ & $\begin{array}{l}49 \\
283 \\
570 \\
902 \\
1098 \\
1262 \\
1389 \\
1494 \\
1765 \\
3081\end{array}$ & $\begin{array}{l}107 \\
327 \\
577 \\
920 \\
1116 \\
1288 \\
1408 \\
1506 \\
2567 \\
3127\end{array}$ & $\begin{array}{l}139 \\
439 \\
719 \\
961 \\
1155 \\
1338 \\
1441 \\
1531 \\
3069 \\
3132\end{array}$ & $\begin{array}{l}178 \\
471 \\
786 \\
1029 \\
1180 \\
1356 \\
1455 \\
1557 \\
3076 \\
3142\end{array}$ \\
\hline $\mathrm{HOCH}_{2} \mathrm{CH}_{2} \mathrm{CH}_{2} \mathrm{CHO} \bullet \mathrm{FA}(\mathrm{PC} 5 \mathrm{~A})$ & $\begin{array}{l}78 \\
223 \\
612 \\
888 \\
1032 \\
1213\end{array}$ & $\begin{array}{l}95 \\
225 \\
615 \\
903 \\
1084 \\
1229\end{array}$ & $\begin{array}{l}112 \\
261 \\
711 \\
943 \\
1095 \\
1274\end{array}$ & $\begin{array}{l}162 \\
435 \\
760 \\
965 \\
1107 \\
1278\end{array}$ & $\begin{array}{l}177 \\
501 \\
861 \\
994 \\
1167 \\
1327\end{array}$ \\
\hline
\end{tabular}




\begin{tabular}{|c|c|c|c|c|c|}
\hline & $\begin{array}{l}1333 \\
1456 \\
1811 \\
3127 \\
3741\end{array}$ & $\begin{array}{l}1352 \\
1467 \\
3073 \\
3144\end{array}$ & $\begin{array}{l}1368 \\
1491 \\
3073 \\
3147\end{array}$ & $\begin{array}{l}1406 \\
1511 \\
3090 \\
3152\end{array}$ & $\begin{array}{l}1417 \\
1538 \\
3123 \\
3215\end{array}$ \\
\hline $\mathrm{HOCH}_{2} \mathrm{CH}_{2} \mathrm{CH}_{2} \mathrm{CHO} \bullet \cdot \mathrm{FA}(\mathrm{RC} 6)$ & $\begin{array}{l}52 \\
181 \\
559 \\
875 \\
1024 \\
1217 \\
1308 \\
1431 \\
1848 \\
3133 \\
3877\end{array}$ & $\begin{array}{l}71 \\
199 \\
695 \\
925 \\
1060 \\
1222 \\
1335 \\
1438 \\
3080 \\
3136\end{array}$ & $\begin{array}{l}93 \\
235 \\
702 \\
941 \\
1089 \\
1254 \\
1375 \\
1491 \\
3093 \\
3150\end{array}$ & $\begin{array}{l}103 \\
407 \\
768 \\
957 \\
1119 \\
1261 \\
1391 \\
1515 \\
3101 \\
3164\end{array}$ & $\begin{array}{l}136 \\
439 \\
828 \\
991 \\
1167 \\
1277 \\
1411 \\
1544 \\
3101 \\
3383\end{array}$ \\
\hline $\mathrm{HOCH}_{2} \mathrm{CH}_{2} \mathrm{CH}_{2} \mathrm{CHO} \bullet \mathrm{FA}$ (TS6) & $\begin{array}{l}-160 \\
164 \\
290 \\
800 \\
941 \\
1109 \\
1326 \\
1436 \\
1669 \\
3143 \\
3954\end{array}$ & $\begin{array}{l}59 \\
185 \\
484 \\
826 \\
980 \\
1160 \\
1352 \\
1487 \\
2017 \\
3151\end{array}$ & $\begin{array}{l}83 \\
218 \\
495 \\
857 \\
1013 \\
1232 \\
1386 \\
1505 \\
2982 \\
3199\end{array}$ & $\begin{array}{l}102 \\
242 \\
660 \\
869 \\
1081 \\
1256 \\
1405 \\
1563 \\
3080 \\
3240\end{array}$ & $\begin{array}{l}142 \\
245 \\
757 \\
917 \\
1094 \\
1293 \\
1421 \\
1661 \\
3125 \\
3304\end{array}$ \\
\hline $\mathrm{HOCH}_{2} \mathrm{CH}_{2} \mathrm{CH}_{2} \mathrm{CHO} \bullet \cdot \mathrm{FA}$ (PC6) & $\begin{array}{l}50 \\
128 \\
278 \\
716 \\
955 \\
1093 \\
1269 \\
1457 \\
1826 \\
3160 \\
3882\end{array}$ & $\begin{array}{l}62 \\
188 \\
467 \\
755 \\
960 \\
1104 \\
1317 \\
1498 \\
3070 \\
3233\end{array}$ & $\begin{array}{l}90 \\
210 \\
516 \\
846 \\
1029 \\
1175 \\
1334 \\
1531 \\
3084 \\
3237\end{array}$ & $\begin{array}{l}105 \\
227 \\
637 \\
856 \\
1040 \\
1229 \\
1407 \\
1665 \\
3110 \\
3266\end{array}$ & $\begin{array}{l}125 \\
230 \\
704 \\
943 \\
1087 \\
1255 \\
1421 \\
1706 \\
3113 \\
3733\end{array}$ \\
\hline $\mathrm{HOCH}_{2} \mathrm{CH}_{2} \mathrm{CH}_{2} \mathrm{CHO} \bullet \cdot \mathrm{HNO}_{3}(\mathrm{RC} 7)$ & $\begin{array}{l}39 \\
152 \\
349 \\
733\end{array}$ & $\begin{array}{l}45 \\
174 \\
499 \\
841\end{array}$ & $\begin{array}{l}77 \\
197 \\
551 \\
845\end{array}$ & $\begin{array}{l}94 \\
237 \\
602 \\
861\end{array}$ & $\begin{array}{l}114 \\
306 \\
677 \\
879\end{array}$ \\
\hline
\end{tabular}




\begin{tabular}{|c|c|c|c|c|c|}
\hline & $\begin{array}{l}957 \\
1104 \\
1348 \\
1437 \\
1791 \\
3066 \\
3816\end{array}$ & $\begin{array}{l}1005 \\
1132 \\
1375 \\
1475 \\
1803 \\
3075\end{array}$ & $\begin{array}{l}1023 \\
1173 \\
1393 \\
1488 \\
3005 \\
3097\end{array}$ & $\begin{array}{l}1052 \\
1230 \\
1411 \\
1490 \\
3052 \\
3117\end{array}$ & $\begin{array}{l}1055 \\
1283 \\
1428 \\
1515 \\
3064 \\
3126\end{array}$ \\
\hline $\mathrm{HOCH}_{2} \mathrm{CH}_{2} \mathrm{CH}_{2} \mathrm{CHO} \bullet \cdot \mathrm{HNO}_{3}(\mathrm{TS} 7)$ & $\begin{array}{l}-532 \\
171 \\
444 \\
821 \\
961 \\
1122 \\
1343 \\
1457 \\
1563 \\
3088 \\
3153\end{array}$ & $\begin{array}{l}51 \\
223 \\
577 \\
863 \\
996 \\
1148 \\
1364 \\
1479 \\
1669 \\
3093\end{array}$ & $\begin{array}{l}65 \\
244 \\
673 \\
877 \\
1022 \\
1207 \\
1376 \\
1497 \\
1708 \\
3135\end{array}$ & $\begin{array}{l}104 \\
357 \\
723 \\
897 \\
1072 \\
1239 \\
1395 \\
1519 \\
3066 \\
3138\end{array}$ & $\begin{array}{l}138 \\
412 \\
741 \\
911 \\
1094 \\
1280 \\
1428 \\
1524 \\
3082 \\
3147\end{array}$ \\
\hline $\mathrm{HOCH}_{2} \mathrm{CH}_{2} \mathrm{CH}_{2} \mathrm{CHO} \cdot \bullet \mathrm{HNO}_{3}(\mathrm{PC} 7)$ & \begin{tabular}{|l}
36 \\
147 \\
569 \\
846 \\
960 \\
1092 \\
1284 \\
1423 \\
1557 \\
3105 \\
3854
\end{tabular} & $\begin{array}{l}54 \\
212 \\
639 \\
864 \\
993 \\
1164 \\
1333 \\
1459 \\
1804 \\
3134\end{array}$ & $\begin{array}{l}79 \\
222 \\
686 \\
882 \\
1025 \\
1213 \\
1362 \\
1490 \\
2888 \\
3138\end{array}$ & $\begin{array}{l}101 \\
437 \\
736 \\
921 \\
1059 \\
1223 \\
1379 \\
1510 \\
3089 \\
3154\end{array}$ & $\begin{array}{l}122 \\
502 \\
803 \\
940 \\
1070 \\
1271 \\
1404 \\
1538 \\
3093 \\
3165\end{array}$ \\
\hline $\mathrm{HOCH}_{2} \mathrm{CH}_{2} \mathrm{CH}_{2} \mathrm{CHO} \cdot \cdot \mathrm{HNO}_{3}(\mathrm{RC} 7 \mathrm{~A})$ & \begin{tabular}{|l}
57 \\
157 \\
312 \\
736 \\
945 \\
1102 \\
1358 \\
1443 \\
1795 \\
3082 \\
3861
\end{tabular} & $\begin{array}{l}68 \\
164 \\
494 \\
808 \\
965 \\
1134 \\
1385 \\
1468 \\
1814 \\
3084\end{array}$ & $\begin{array}{l}76 \\
206 \\
564 \\
843 \\
1018 \\
1173 \\
1394 \\
1488 \\
3047 \\
3102\end{array}$ & $\begin{array}{l}108 \\
229 \\
607 \\
859 \\
1041 \\
1234 \\
1425 \\
1496 \\
3053 \\
3109\end{array}$ & $\begin{array}{l}117 \\
303 \\
675 \\
868 \\
1055 \\
1285 \\
1432 \\
1511 \\
3057 \\
3131\end{array}$ \\
\hline $\mathrm{HOCH}_{2} \mathrm{CH}_{2} \mathrm{CH}_{2} \mathrm{CHO} \cdot \bullet \mathrm{HNO}_{3}(\mathrm{TS} 7 \mathrm{~A})$ & $\begin{array}{l}-369 \\
173\end{array}$ & $\begin{array}{l}49 \\
231\end{array}$ & $\begin{array}{l}68 \\
270\end{array}$ & $\begin{array}{l}103 \\
384\end{array}$ & $\begin{array}{l}146 \\
408\end{array}$ \\
\hline
\end{tabular}




\begin{tabular}{|c|c|c|c|c|c|}
\hline & $\begin{array}{l}474 \\
836 \\
967 \\
1134 \\
1344 \\
1465 \\
1577 \\
3093 \\
3250\end{array}$ & $\begin{array}{l}552 \\
865 \\
1034 \\
1144 \\
1358 \\
1481 \\
1678 \\
3128\end{array}$ & $\begin{array}{l}639 \\
883 \\
1053 \\
1191 \\
1382 \\
1496 \\
1712 \\
3136\end{array}$ & $\begin{array}{l}738 \\
904 \\
1079 \\
1240 \\
1391 \\
1514 \\
3069 \\
3153\end{array}$ & $\begin{array}{l}778 \\
915 \\
1109 \\
1279 \\
1417 \\
1533 \\
3080 \\
3166\end{array}$ \\
\hline $\mathrm{HOCH}_{2} \mathrm{CH}_{2} \mathrm{CH}_{2} \mathrm{CHO} \cdot \bullet \mathrm{HNO}_{3}(\mathrm{PC} 7 \mathrm{~A})$ & $\begin{array}{l}61 \\
187 \\
543 \\
848 \\
962 \\
1102 \\
1326 \\
1419 \\
1533 \\
3089 \\
3821\end{array}$ & $\begin{array}{l}95 \\
221 \\
611 \\
857 \\
1004 \\
1165 \\
1326 \\
1468 \\
1793 \\
3126\end{array}$ & $\begin{array}{l}102 \\
276 \\
680 \\
885 \\
1040 \\
1207 \\
1352 \\
1490 \\
2940 \\
3144\end{array}$ & $\begin{array}{l}139 \\
432 \\
736 \\
899 \\
1059 \\
1229 \\
1366 \\
1509 \\
3063 \\
3152\end{array}$ & $\begin{array}{l}158 \\
477 \\
757 \\
944 \\
1087 \\
1273 \\
1401 \\
1510 \\
3076 \\
3162\end{array}$ \\
\hline 2-OH-THF••HNO 3 (RC8) & $\begin{array}{l}43 \\
137 \\
550 \\
827 \\
955 \\
1117 \\
1276 \\
1431 \\
1530 \\
3119 \\
3884\end{array}$ & $\begin{array}{l}77 \\
196 \\
631 \\
843 \\
994 \\
1168 \\
1321 \\
1435 \\
1794 \\
3140\end{array}$ & $\begin{array}{l}92 \\
233 \\
666 \\
870 \\
1027 \\
1222 \\
1333 \\
1460 \\
3080 \\
3148\end{array}$ & $\begin{array}{l}109 \\
342 \\
730 \\
915 \\
1044 \\
1243 \\
1379 \\
1487 \\
3084 \\
3151\end{array}$ & $\begin{array}{l}126 \\
441 \\
775 \\
936 \\
1071 \\
1260 \\
1410 \\
1506 \\
3109 \\
3162\end{array}$ \\
\hline 2-OH-THF•• $\mathrm{HNO}_{3}(\mathrm{TS} 8)$ & $\begin{array}{l}-475 \\
108 \\
262 \\
722 \\
880 \\
1031 \\
1238 \\
1433 \\
1631 \\
3154 \\
3958\end{array}$ & $\begin{array}{l}31 \\
142 \\
466 \\
768 \\
902 \\
1087 \\
1282 \\
1444 \\
1663 \\
3184\end{array}$ & $\begin{array}{l}52 \\
201 \\
496 \\
781 \\
939 \\
1095 \\
1314 \\
1497 \\
1724 \\
3186\end{array}$ & $\begin{array}{l}64 \\
224 \\
592 \\
789 \\
949 \\
1133 \\
1337 \\
1508 \\
3077 \\
3278\end{array}$ & $\begin{array}{l}93 \\
233 \\
688 \\
852 \\
1015 \\
1216 \\
1353 \\
1533 \\
3107 \\
3496\end{array}$ \\
\hline
\end{tabular}




\begin{tabular}{|c|c|c|c|c|c|}
\hline 2-OH-THF••HNO ${ }_{3}(\mathrm{PC} 8)$ & $\begin{array}{l}37 \\
115 \\
384 \\
702 \\
860 \\
1050 \\
1228 \\
1422 \\
1716 \\
3105 \\
3905\end{array}$ & $\begin{array}{l}53 \\
131 \\
461 \\
734 \\
942 \\
1078 \\
1267 \\
1508 \\
1797 \\
3137\end{array}$ & $\begin{array}{l}57 \\
177 \\
497 \\
754 \\
944 \\
1095 \\
1317 \\
1536 \\
2849 \\
3243\end{array}$ & $\begin{array}{l}67 \\
211 \\
677 \\
845 \\
956 \\
1124 \\
1336 \\
1539 \\
3069 \\
3270\end{array}$ & $\begin{array}{l}101 \\
285 \\
693 \\
846 \\
1028 \\
1169 \\
1416 \\
1649 \\
3078 \\
3611\end{array}$ \\
\hline trans $-\mathrm{H}_{2} \mathrm{SO}_{4}$ & $\begin{array}{l}261 \\
564 \\
1176\end{array}$ & $\begin{array}{l}342 \\
577 \\
1267\end{array}$ & $\begin{array}{l}386 \\
889 \\
1510\end{array}$ & $\begin{array}{l}448 \\
937 \\
3833\end{array}$ & $\begin{array}{l}512 \\
1156 \\
3844\end{array}$ \\
\hline $\mathrm{HOCH}_{2} \mathrm{CH}_{2} \mathrm{CH}_{2} \mathrm{CHO} \cdot \bullet$ trans- $\mathrm{H}_{2} \mathrm{SO}_{4}$ (RC9) & $\begin{array}{l}41 \\
160 \\
316 \\
523 \\
779 \\
1001 \\
1136 \\
1300 \\
1435 \\
1527 \\
3051 \\
3837\end{array}$ & $\begin{array}{l}58 \\
181 \\
380 \\
552 \\
867 \\
1016 \\
1153 \\
1346 \\
1442 \\
1779 \\
3084 \\
3845\end{array}$ & $\begin{array}{l}90 \\
229 \\
404 \\
565 \\
908 \\
1025 \\
1217 \\
1366 \\
1451 \\
2494 \\
3099\end{array}$ & $\begin{array}{l}103 \\
243 \\
437 \\
566 \\
913 \\
1087 \\
1234 \\
1384 \\
1465 \\
3036 \\
3107\end{array}$ & $\begin{array}{l}133 \\
269 \\
476 \\
585 \\
985 \\
1117 \\
1242 \\
1396 \\
1486 \\
3049 \\
3116\end{array}$ \\
\hline $\mathrm{HOCH}_{2} \mathrm{CH}_{2} \mathrm{CH}_{2} \mathrm{CHO} \bullet$ trans- $\mathrm{H}_{2} \mathrm{SO}_{4}$ (TS9) & $\begin{array}{l}-196 \\
154 \\
371 \\
572 \\
845 \\
1003 \\
1150 \\
1312 \\
1395 \\
1546 \\
3074 \\
3480\end{array}$ & $\begin{array}{l}62 \\
207 \\
435 \\
579 \\
898 \\
1024 \\
1171 \\
1332 \\
1442 \\
1616 \\
3130 \\
3864\end{array}$ & $\begin{array}{l}80 \\
239 \\
461 \\
604 \\
906 \\
1071 \\
1212 \\
1366 \\
1474 \\
1802 \\
3132\end{array}$ & $\begin{array}{l}116 \\
283 \\
469 \\
649 \\
932 \\
1102 \\
1223 \\
1373 \\
1493 \\
3059 \\
3143\end{array}$ & $\begin{array}{l}124 \\
328 \\
518 \\
832 \\
936 \\
1140 \\
1293 \\
1385 \\
1528 \\
3062 \\
3180\end{array}$ \\
\hline $\mathrm{HOCH}_{2} \mathrm{CH}_{2} \mathrm{CH}_{2} \mathrm{CHO} \cdot$ •trans- $\mathrm{H}_{2} \mathrm{SO}_{4}$ (PC9) & $\begin{array}{l}35 \\
148 \\
414 \\
569\end{array}$ & $\begin{array}{l}75 \\
205 \\
434 \\
584\end{array}$ & $\begin{array}{l}86 \\
228 \\
541 \\
543\end{array}$ & $\begin{array}{l}115 \\
287 \\
552 \\
669\end{array}$ & $\begin{array}{l}142 \\
402 \\
562 \\
838\end{array}$ \\
\hline
\end{tabular}




\begin{tabular}{|c|c|c|c|c|c|}
\hline & $\begin{array}{l}860 \\
1008 \\
1152 \\
1279 \\
1401 \\
1510 \\
3091 \\
3745\end{array}$ & $\begin{array}{l}910 \\
1038 \\
1199 \\
1314 \\
1419 \\
1538 \\
3112 \\
3846\end{array}$ & $\begin{array}{l}919 \\
1073 \\
1227 \\
1343 \\
1432 \\
2484 \\
3131\end{array}$ & $\begin{array}{l}949 \\
1111 \\
1232 \\
1372 \\
1479 \\
3073 \\
3141\end{array}$ & $\begin{array}{l}973 \\
1136 \\
1241 \\
1389 \\
1495 \\
3082 \\
3152\end{array}$ \\
\hline $\mathrm{HOCH}_{2} \mathrm{CH}_{2} \mathrm{CH}_{2} \mathrm{CHO} \cdot \bullet$ trans- $\mathrm{H}_{2} \mathrm{SO}_{4}(\mathrm{RC} 9 \mathrm{~A})$ & \begin{tabular}{|l}
32 \\
142 \\
305 \\
543 \\
846 \\
984 \\
1136 \\
1283 \\
1415 \\
1514 \\
3065 \\
3764
\end{tabular} & $\begin{array}{l}43 \\
167 \\
360 \\
571 \\
862 \\
1007 \\
1164 \\
1345 \\
1435 \\
1789 \\
3084 \\
3840\end{array}$ & $\begin{array}{l}53 \\
205 \\
404 \\
581 \\
882 \\
1025 \\
1178 \\
1369 \\
1475 \\
2803 \\
3095\end{array}$ & $\begin{array}{l}84 \\
243 \\
442 \\
604 \\
884 \\
1059 \\
1229 \\
1373 \\
1483 \\
3051 \\
3117\end{array}$ & $\begin{array}{l}108 \\
268 \\
506 \\
618 \\
955 \\
1106 \\
1245 \\
1402 \\
1489 \\
3064 \\
3126\end{array}$ \\
\hline $\mathrm{HOCH}_{2} \mathrm{CH}_{2} \mathrm{CH}_{2} \mathrm{CHO} \cdot \bullet$ trans $-\mathrm{H}_{2} \mathrm{SO}_{4}(\mathrm{TS} 9 \mathrm{~A})$ & \begin{tabular}{|l}
-367 \\
146 \\
359 \\
578 \\
858 \\
962 \\
1136 \\
1330 \\
1406 \\
1516 \\
3088 \\
3270
\end{tabular} & $\begin{array}{l}23 \\
187 \\
400 \\
597 \\
878 \\
1027 \\
1161 \\
1350 \\
1437 \\
1523 \\
3128 \\
3858\end{array}$ & $\begin{array}{l}54 \\
202 \\
413 \\
636 \\
892 \\
1074 \\
1199 \\
1368 \\
1448 \\
1690 \\
3136\end{array}$ & $\begin{array}{l}82 \\
228 \\
462 \\
689 \\
901 \\
1087 \\
1235 \\
1371 \\
1480 \\
3070 \\
3150\end{array}$ & $\begin{array}{l}127 \\
338 \\
560 \\
793 \\
908 \\
1111 \\
1276 \\
1387 \\
1498 \\
3080 \\
3163\end{array}$ \\
\hline $\mathrm{HOCH}_{2} \mathrm{CH}_{2} \mathrm{CH}_{2} \mathrm{CHO} \cdot \bullet$ trans- $\mathrm{H}_{2} \mathrm{SO}_{4}(\mathrm{PC} 9 \mathrm{~A})$ & \begin{tabular}{|l}
25 \\
140 \\
436 \\
582 \\
878 \\
978 \\
1059 \\
1272 \\
1406 \\
1526 \\
3097
\end{tabular} & $\begin{array}{l}34 \\
207 \\
455 \\
640 \\
886 \\
1008 \\
1169 \\
1298 \\
1432 \\
1535 \\
3127\end{array}$ & $\begin{array}{l}71 \\
220 \\
519 \\
645 \\
809 \\
1026 \\
1217 \\
1332 \\
1463 \\
2615 \\
3130\end{array}$ & $\begin{array}{l}93 \\
306 \\
548 \\
801 \\
940 \\
1071 \\
1224 \\
1357 \\
1486 \\
3083 \\
3152\end{array}$ & $\begin{array}{l}107 \\
398 \\
574 \\
855 \\
959 \\
1086 \\
1253 \\
1377 \\
1507 \\
3085 \\
3162\end{array}$ \\
\hline
\end{tabular}




\begin{tabular}{|c|c|c|c|c|c|}
\hline & 3788 & 3849 & & & \\
\hline \multirow[t]{12}{*}{ 2-OH-THF••trans- $\mathrm{H}_{2} \mathrm{SO}_{4}(\mathrm{RC} 10)$} & 46 & 58 & 68 & 89 & 109 \\
\hline & 110 & 185 & 253 & 285 & 398 \\
\hline & 325 & 389 & 441 & 446 & 540 \\
\hline & 569 & 578 & 636 & 781 & 865 \\
\hline & 866 & 878 & 885 & 939 & 960 \\
\hline & 962 & 969 & 1004 & 1057 & 1126 \\
\hline & 1162 & 1164 & 1199 & 1216 & 1248 \\
\hline & 1258 & 1268 & 1329 & 1351 & 1356 \\
\hline & 1380 & 1407 & 1444 & 1489 & 1504 \\
\hline & 1514 & 1541 & 3016 & 3070 & 3082 \\
\hline & 3096 & 3128 & 3130 & 3149 & 3156 \\
\hline & 3851 & 3889 & & & \\
\hline \multirow[t]{12}{*}{ 2-OH-THF••trans- $\mathrm{H}_{2} \mathrm{SO}_{4}(\mathrm{TS} 10)$} & $\mid-953$ & 35 & 46 & 68 & 89 \\
\hline & 117 & 143 & 163 & 186 & 197 \\
\hline & 232 & 240 & 374 & 391 & 405 \\
\hline & 467 & 532 & 556 & 585 & 613 \\
\hline & 621 & 682 & 780 & 848 & 853 \\
\hline & 872 & 937 & 943 & 1014 & 1023 \\
\hline & 1089 & 1096 & 1106 & 1152 & 1207 \\
\hline & 1234 & 1260 & 1288 & 1320 & 1336 \\
\hline & 1355 & 1384 & 1422 & 1452 & 1500 \\
\hline & 1526 & 1636 & 1645 & 3078 & 3119 \\
\hline & 3139 & 3176 & 3184 & 3254 & 3739 \\
\hline & 3871 & 3967 & & & \\
\hline \multirow[t]{12}{*}{ 2-OH-THF••trans- $\mathrm{H}_{2} \mathrm{SO}_{4}(\mathrm{PC} 10)$} & 28 & 48 & 59 & 80 & 91 \\
\hline & 119 & 131 & 188 & 213 & 262 \\
\hline & 301 & 333 & 381 & 451 & 461 \\
\hline & 485 & 546 & 569 & 583 & 699 \\
\hline & 746 & 753 & 845 & 852 & 884 \\
\hline & 939 & 943 & 960 & 991 & 1030 \\
\hline & 1081 & 1194 & 1099 & 1169 & 1169 \\
\hline & 1225 & 1247 & 1267 & 1316 & 1335 \\
\hline & 1382 & 1416 & 1482 & 1494 & 1527 \\
\hline & 1653 & 1721 & 2657 & 3066 & 3077 \\
\hline & 3108 & 3160 & 3237 & 3267 & 3488 \\
\hline & 3841 & 3931 & & & \\
\hline \multirow[t]{3}{*}{ cis- $\mathrm{H}_{2} \mathrm{SO}_{4}$} & 138 & 354 & 378 & 432 & 514 \\
\hline & 562 & 576 & 885 & 936 & 1134 \\
\hline & 1170 & 1264 & 1512 & 3845 & 3854 \\
\hline \multirow[t]{2}{*}{$\mathrm{HOCH}_{2} \mathrm{CH}_{2} \mathrm{CH}_{2} \mathrm{CHO} \cdot{ }^{-}$cis- $\mathrm{H}_{2} \mathrm{SO}_{4}$ (RC9B) } & 46 & 52 & 87 & 98 & 128 \\
\hline & 160 & 183 & 243 & 249 & 276 \\
\hline
\end{tabular}




\begin{tabular}{|c|c|c|c|c|c|}
\hline & $\begin{array}{l}316 \\
536 \\
779 \\
995 \\
1135 \\
1298 \\
1433 \\
1524 \\
3052 \\
3832\end{array}$ & $\begin{array}{l}381 \\
550 \\
867 \\
1024 \\
1155 \\
1346 \\
1442 \\
1780 \\
3081 \\
3836\end{array}$ & $\begin{array}{l}428 \\
564 \\
909 \\
1031 \\
1216 \\
1369 \\
1450 \\
2532 \\
3102\end{array}$ & $\begin{array}{l}436 \\
567 \\
913 \\
1087 \\
1233 \\
1384 \\
1464 \\
3034 \\
3108\end{array}$ & $\begin{array}{l}478 \\
584 \\
950 \\
1118 \\
1239 \\
1407 \\
1486 \\
3050 \\
3118\end{array}$ \\
\hline $\mathrm{HOCH}_{2} \mathrm{CH}_{2} \mathrm{CH}_{2} \mathrm{CHO} \cdot \bullet$ cis- $\mathrm{H}_{2} \mathrm{SO}_{4}$ (TS9B) & $\begin{array}{l}-209 \\
153 \\
373 \\
565 \\
843 \\
981 \\
1158 \\
1305 \\
1399 \\
1547 \\
3074 \\
3464\end{array}$ & $\begin{array}{l}71 \\
206 \\
443 \\
578 \\
899 \\
1025 \\
1167 \\
1330 \\
1441 \\
1602 \\
3130 \\
3852\end{array}$ & $\begin{array}{l}79 \\
229 \\
461 \\
601 \\
907 \\
1070 \\
1212 \\
1368 \\
1473 \\
1771 \\
3132\end{array}$ & $\begin{array}{l}116 \\
282 \\
472 \\
659 \\
930 \\
1101 \\
1224 \\
1377 \\
1493 \\
3059 \\
3142\end{array}$ & $\begin{array}{l}121 \\
322 \\
517 \\
833 \\
936 \\
1136 \\
1292 \\
1388 \\
1526 \\
3062 \\
3179\end{array}$ \\
\hline $\mathrm{HOCH}_{2} \mathrm{CH}_{2} \mathrm{CH}_{2} \mathrm{CHO} \cdot \bullet$ cis- $\mathrm{H}_{2} \mathrm{SO}_{4}(\mathrm{PC} 9 \mathrm{~B})$ & $\begin{array}{l}35 \\
149 \\
414 \\
568 \\
860 \\
1007 \\
1160 \\
1279 \\
1404 \\
1509 \\
3083 \\
3748\end{array}$ & $\begin{array}{l}73 \\
204 \\
442 \\
584 \\
910 \\
1038 \\
1198 \\
1314 \\
1425 \\
1535 \\
3109 \\
3842\end{array}$ & $\begin{array}{l}88 \\
259 \\
542 \\
646 \\
916 \\
1072 \\
1226 \\
1341 \\
1434 \\
2472 \\
3128\end{array}$ & $\begin{array}{l}115 \\
297 \\
553 \\
668 \\
948 \\
1111 \\
1229 \\
1371 \\
1480 \\
3072 \\
3139\end{array}$ & $\begin{array}{l}134 \\
401 \\
562 \\
835 \\
973 \\
1137 \\
1236 \\
1391 \\
1493 \\
3079 \\
3149\end{array}$ \\
\hline 2-OH-THF••OH (TS11) & $\begin{array}{l}-660 \\
321 \\
625 \\
948 \\
1125 \\
1296 \\
1484 \\
3096 \\
3785\end{array}$ & $\begin{array}{l}28 \\
328 \\
779 \\
959 \\
1186 \\
1337 \\
1500 \\
3100 \\
3873\end{array}$ & $\begin{array}{l}79 \\
410 \\
845 \\
1014 \\
1218 \\
1344 \\
1537 \\
3126\end{array}$ & $\begin{array}{l}130 \\
515 \\
884 \\
1027 \\
1237 \\
1390 \\
1885 \\
3156\end{array}$ & $\begin{array}{l}199 \\
614 \\
902 \\
1087 \\
1279 \\
1415 \\
3062 \\
3172\end{array}$ \\
\hline
\end{tabular}




\begin{tabular}{|c|c|c|c|c|c|}
\hline 2-OH-THF ••OH (RC11) & $\begin{array}{l}32 \\
257 \\
674 \\
943 \\
1163 \\
1329 \\
1486 \\
3099 \\
3550\end{array}$ & $\begin{array}{l}68 \\
389 \\
789 \\
961 \\
1210 \\
1352 \\
1503 \\
3104 \\
3817\end{array}$ & $\begin{array}{c}116 \\
430 \\
867 \\
1021 \\
1217 \\
1374 \\
1537 \\
3122\end{array}$ & $\begin{array}{c}168 \\
512 \\
877 \\
1063 \\
1268 \\
1407 \\
3073 \\
3157\end{array}$ & $\begin{array}{l}189 \\
631 \\
939 \\
1107 \\
1279 \\
1459 \\
3084 \\
3170\end{array}$ \\
\hline 2-OH-THF••OH (PC11) & $\begin{array}{l}44 \\
239 \\
612 \\
903 \\
1149 \\
1333 \\
1503 \\
3094 \\
3793\end{array}$ & $\begin{array}{c}68 \\
268 \\
659 \\
941 \\
1191 \\
1355 \\
1525 \\
3119 \\
3949\end{array}$ & $\begin{array}{c}138 \\
424 \\
717 \\
1009 \\
1216 \\
1401 \\
1626 \\
3145\end{array}$ & $\begin{array}{c}194 \\
504 \\
827 \\
1073 \\
1269 \\
1451 \\
3048 \\
3155\end{array}$ & $\begin{array}{c}227 \\
587 \\
896 \\
1085 \\
1298 \\
1484 \\
3076 \\
3675\end{array}$ \\
\hline 2-OH-THF••OH (P11) & $\begin{array}{l}59 \\
611 \\
940 \\
1195 \\
1339 \\
1527 \\
3146\end{array}$ & $\begin{array}{l}234 \\
653 \\
1005 \\
1213 \\
1399 \\
3050 \\
3156\end{array}$ & $\begin{array}{l}269 \\
822 \\
1065 \\
1264 \\
1418 \\
3077 \\
3882\end{array}$ & $\begin{array}{l}404 \\
899 \\
1076 \\
1290 \\
1483 \\
3095\end{array}$ & $\begin{array}{l}515 \\
905 \\
1152 \\
1305 \\
1501 \\
3118\end{array}$ \\
\hline 2-OH-THF••OH (TS12) & \begin{tabular}{|l}
-1591 \\
305 \\
660 \\
948 \\
1137 \\
1304 \\
1485 \\
3037 \\
3158
\end{tabular} & $\begin{array}{l}70 \\
412 \\
805 \\
956 \\
1158 \\
1322 \\
1499 \\
3082 \\
3786\end{array}$ & $\begin{array}{l}101 \\
428 \\
864 \\
980 \\
1213 \\
1348 \\
1532 \\
3091\end{array}$ & $\begin{array}{l}156 \\
568 \\
891 \\
1053 \\
1214 \\
1371 \\
1532 \\
3127\end{array}$ & $\begin{array}{l}242 \\
587 \\
911 \\
1124 \\
1276 \\
1403 \\
2999 \\
3147\end{array}$ \\
\hline 2-OH-THF••OH (RC12) & \begin{tabular}{|l}
45 \\
399 \\
657 \\
956 \\
1180 \\
1334 \\
1485
\end{tabular} & $\begin{array}{c}109 \\
423 \\
738 \\
973 \\
1209 \\
1364 \\
1504\end{array}$ & $\begin{array}{l}154 \\
446 \\
867 \\
1049 \\
1218 \\
1384 \\
1540\end{array}$ & $\begin{array}{l}210 \\
548 \\
883 \\
1110 \\
1275 \\
1414 \\
3034\end{array}$ & $\begin{array}{l}253 \\
569 \\
909 \\
1124 \\
1311 \\
1467 \\
3048\end{array}$ \\
\hline
\end{tabular}




\begin{tabular}{|c|c|c|c|c|c|}
\hline & $\begin{array}{l}3073 \\
3581\end{array}$ & $\begin{array}{l}3100 \\
3826\end{array}$ & 3126 & 3142 & 3148 \\
\hline 2-OH-THF••OH (PC12) & $\begin{array}{l}68 \\
268 \\
578 \\
949 \\
1146 \\
1297 \\
1495 \\
3071 \\
3826\end{array}$ & $\begin{array}{l}106 \\
300 \\
644 \\
975 \\
1185 \\
1308 \\
1534 \\
3092 \\
3963\end{array}$ & $\begin{array}{l}139 \\
359 \\
842 \\
1021 \\
1200 \\
1345 \\
1637 \\
3127\end{array}$ & $\begin{array}{l}155 \\
505 \\
888 \\
1049 \\
1224 \\
1409 \\
2952 \\
3138\end{array}$ & $\begin{array}{l}231 \\
553 \\
897 \\
1113 \\
1280 \\
1485 \\
3064 \\
3167\end{array}$ \\
\hline 2-OH-THF••OH (P12) & $\begin{array}{l}87 \\
700 \\
963 \\
1194 \\
1321 \\
1526 \\
3136\end{array}$ & $\begin{array}{l}247 \\
845 \\
1005 \\
1217 \\
1355 \\
3037 \\
3145\end{array}$ & $\begin{array}{l}356 \\
860 \\
1047 \\
1226 \\
1396 \\
3062 \\
3169\end{array}$ & $\begin{array}{l}557 \\
909 \\
1126 \\
1278 \\
1488 \\
3082\end{array}$ & $\begin{array}{l}600 \\
940 \\
1137 \\
1302 \\
1503 \\
3106\end{array}$ \\
\hline 2-OH-THF••OH (TS13) & $\begin{array}{l}-1026 \\
325 \\
752 \\
946 \\
1138 \\
1262 \\
1439 \\
3093 \\
3772\end{array}$ & $\begin{array}{l}73 \\
346 \\
807 \\
964 \\
1161 \\
1295 \\
1486 \\
3097 \\
3885\end{array}$ & $\begin{array}{l}106 \\
362 \\
852 \\
1020 \\
1191 \\
1319 \\
1534 \\
3128\end{array}$ & $\begin{array}{l}125 \\
522 \\
905 \\
1046 \\
1208 \\
1368 \\
1599 \\
3150\end{array}$ & $\begin{array}{l}189 \\
643 \\
942 \\
1069 \\
1248 \\
1408 \\
3083 \\
3165\end{array}$ \\
\hline 2-OH-THF••OH (RC13) & $\begin{array}{l}45 \\
327 \\
650 \\
947 \\
1158 \\
1330 \\
1491 \\
3094 \\
3615\end{array}$ & $\begin{array}{l}77 \\
422 \\
785 \\
956 \\
1206 \\
1348 \\
1506 \\
3107 \\
3890\end{array}$ & $\begin{array}{l}100 \\
434 \\
862 \\
1027 \\
1220 \\
1377 \\
1537 \\
3128\end{array}$ & $\begin{array}{l}178 \\
529 \\
883 \\
1056 \\
1259 \\
1413 \\
3081 \\
3158\end{array}$ & $\begin{array}{l}211 \\
599 \\
938 \\
1108 \\
1271 \\
1442 \\
3083 \\
3167\end{array}$ \\
\hline 2-OH-THF••OH (PC13) & $\begin{array}{l}36 \\
227 \\
561 \\
932 \\
1082 \\
1315\end{array}$ & $\begin{array}{l}74 \\
277 \\
588 \\
944 \\
1119 \\
1319\end{array}$ & $\begin{array}{l}135 \\
319 \\
652 \\
962 \\
1203 \\
1373\end{array}$ & $\begin{array}{l}159 \\
357 \\
806 \\
1028 \\
1212 \\
1405\end{array}$ & $\begin{array}{l}171 \\
464 \\
865 \\
1053 \\
1264 \\
1443\end{array}$ \\
\hline
\end{tabular}




\begin{tabular}{|c|c|c|c|c|c|}
\hline & $\begin{array}{l}1474 \\
3073 \\
3872\end{array}$ & $\begin{array}{l}1520 \\
3101 \\
3957\end{array}$ & $\begin{array}{l}1631 \\
3153\end{array}$ & $\begin{array}{l}3036 \\
3272\end{array}$ & $\begin{array}{l}3059 \\
3762\end{array}$ \\
\hline 2-OH-THF••OH (P13) & $\begin{array}{l}126 \\
557 \\
950 \\
1140 \\
1316 \\
1520 \\
3135\end{array}$ & $\begin{array}{l}200 \\
651 \\
972 \\
1198 \\
1371 \\
3023 \\
3252\end{array}$ & $\begin{array}{l}264 \\
812 \\
1025 \\
1208 \\
1406 \\
3055 \\
3879\end{array}$ & $\begin{array}{l}335 \\
863 \\
1054 \\
1259 \\
1441 \\
3075\end{array}$ & $\begin{array}{l}468 \\
935 \\
1074 \\
1307 \\
1474 \\
3086\end{array}$ \\
\hline 2-OH-THF••OH (TS14) & $\begin{array}{l}-1006 \\
336 \\
692 \\
955 \\
1129 \\
1282 \\
1422 \\
3075 \\
3774\end{array}$ & $\begin{array}{l}55 \\
387 \\
813 \\
979 \\
1185 \\
1306 \\
1456 \\
3101 \\
3905\end{array}$ & $\begin{array}{l}119 \\
430 \\
833 \\
1039 \\
1219 \\
1327 \\
1485 \\
3136\end{array}$ & $\begin{array}{l}186 \\
554 \\
875 \\
1069 \\
1231 \\
1373 \\
1524 \\
3142\end{array}$ & $\begin{array}{l}254 \\
592 \\
928 \\
1108 \\
1259 \\
1406 \\
3054 \\
3147\end{array}$ \\
\hline 2-OH-THF••OH (RC14) & $\begin{array}{l}69 \\
361 \\
654 \\
952 \\
1165 \\
1314 \\
1495 \\
3104 \\
3601\end{array}$ & $\begin{array}{l}114 \\
426 \\
797 \\
996 \\
1218 \\
1331 \\
1516 \\
3124 \\
3879\end{array}$ & $\begin{array}{l}125 \\
472 \\
825 \\
1031 \\
1238 \\
1373 \\
1536 \\
3136\end{array}$ & $\begin{array}{l}191 \\
551 \\
875 \\
1061 \\
1258 \\
1411 \\
3046 \\
3149\end{array}$ & $\begin{array}{l}240 \\
637 \\
929 \\
1122 \\
1271 \\
1436 \\
3097 \\
3163\end{array}$ \\
\hline 2-OH-THF••OH (PC14) & $\begin{array}{l}75 \\
220 \\
557 \\
937 \\
1050 \\
1307 \\
1473 \\
3083 \\
3879\end{array}$ & $\begin{array}{l}127 \\
277 \\
617 \\
946 \\
1143 \\
1318 \\
1534 \\
3106 \\
3968\end{array}$ & $\begin{array}{l}143 \\
369 \\
664 \\
970 \\
1203 \\
1367 \\
1651 \\
3149\end{array}$ & $\begin{array}{l}195 \\
385 \\
818 \\
1030 \\
1212 \\
1403 \\
3014 \\
3247\end{array}$ & $\begin{array}{l}211 \\
466 \\
859 \\
1034 \\
1263 \\
1440 \\
3080 \\
3757\end{array}$ \\
\hline 2-OH-THF••OH (TS15) & $\begin{array}{l}-539 \\
193 \\
732 \\
957 \\
1158\end{array}$ & $\begin{array}{l}28 \\
347 \\
801 \\
992 \\
1179\end{array}$ & $\begin{array}{l}49 \\
401 \\
838 \\
1031 \\
1220\end{array}$ & $\begin{array}{l}106 \\
538 \\
888 \\
1057 \\
1234\end{array}$ & $\begin{array}{l}122 \\
623 \\
936 \\
1118 \\
1262\end{array}$ \\
\hline
\end{tabular}




\begin{tabular}{|c|c|c|c|c|c|}
\hline & $\begin{array}{l}1288 \\
1435 \\
3095 \\
3801\end{array}$ & $\begin{array}{l}1320 \\
1487 \\
3106 \\
3886\end{array}$ & $\begin{array}{l}1345 \\
1516 \\
3134\end{array}$ & $\begin{array}{l}1372 \\
1594 \\
3141\end{array}$ & $\begin{array}{l}1409 \\
3056 \\
3164\end{array}$ \\
\hline 2-OH-THF••OH (RC15) & $\begin{array}{l}38 \\
189 \\
625 \\
953 \\
1161 \\
1326 \\
1479 \\
3085 \\
3780\end{array}$ & $\begin{array}{l}48 \\
218 \\
785 \\
961 \\
1196 \\
1349 \\
1500 \\
3095 \\
3891\end{array}$ & $\begin{array}{l}93 \\
315 \\
874 \\
1012 \\
1214 \\
1373 \\
1537 \\
3118\end{array}$ & $\begin{array}{l}103 \\
420 \\
875 \\
1050 \\
1253 \\
1406 \\
3071 \\
3141\end{array}$ & $\begin{array}{l}136 \\
511 \\
941 \\
1129 \\
1269 \\
1441 \\
3078 \\
3163\end{array}$ \\
\hline 2-OH-THF••OH (PC15) & $\begin{array}{l}31 \\
207 \\
515 \\
944 \\
1073 \\
1310 \\
1468 \\
3077 \\
3884\end{array}$ & $\begin{array}{l}86 \\
302 \\
550 \\
955 \\
1122 \\
1326 \\
1505 \\
3102 \\
3934\end{array}$ & $\begin{array}{l}128 \\
346 \\
743 \\
962 \\
1198 \\
1362 \\
1646 \\
3111\end{array}$ & $\begin{array}{l}161 \\
358 \\
804 \\
981 \\
1217 \\
1407 \\
3007 \\
3261\end{array}$ & $\begin{array}{l}185 \\
421 \\
870 \\
1043 \\
1245 \\
1438 \\
3046 \\
3807\end{array}$ \\
\hline 2-OH-THF••OH (P14) & $\begin{array}{l}148 \\
538 \\
960 \\
1141 \\
1332 \\
1503 \\
3108\end{array}$ & $\begin{array}{l}165 \\
748 \\
971 \\
1193 \\
1363 \\
3004 \\
3271\end{array}$ & $\begin{array}{l}255 \\
812 \\
974 \\
1209 \\
1405 \\
3018 \\
3902\end{array}$ & $\begin{array}{l}376 \\
866 \\
1047 \\
1238 \\
1440 \\
3061\end{array}$ & $\begin{array}{l}409 \\
951 \\
1067 \\
1311 \\
1466 \\
3095\end{array}$ \\
\hline 2-OH-THF••OH (TS16) & $\begin{array}{l}-623 \\
196 \\
752 \\
956 \\
1142 \\
1270 \\
1441 \\
3077 \\
3802\end{array}$ & $\begin{array}{l}48 \\
340 \\
811 \\
975 \\
1166 \\
1290 \\
1476 \\
3100 \\
3992\end{array}$ & $\begin{array}{l}64 \\
412 \\
871 \\
1028 \\
1209 \\
1331 \\
1525 \\
3113\end{array}$ & $\begin{array}{l}129 \\
523 \\
890 \\
1042 \\
1226 \\
1369 \\
1590 \\
3129\end{array}$ & $\begin{array}{l}177 \\
595 \\
930 \\
1096 \\
1247 \\
1405 \\
3072 \\
3151\end{array}$ \\
\hline 2-OH-THF••OH (PC16) & $\begin{array}{l}58 \\
231 \\
515 \\
953\end{array}$ & $\begin{array}{l}109 \\
291 \\
572 \\
953\end{array}$ & $\begin{array}{l}133 \\
317 \\
748 \\
968\end{array}$ & $\begin{array}{l}194 \\
389 \\
814 \\
984\end{array}$ & $\begin{array}{l}200 \\
443 \\
869 \\
1044\end{array}$ \\
\hline
\end{tabular}




\begin{tabular}{|c|c|c|c|c|c|}
\hline & $\begin{array}{l}1052 \\
1312 \\
1473 \\
3080 \\
3885\end{array}$ & $\begin{array}{l}1142 \\
1327 \\
1512 \\
3098 \\
3964\end{array}$ & $\begin{array}{l}1184 \\
1367 \\
1653 \\
3127\end{array}$ & $\begin{array}{l}1218 \\
1408 \\
3028 \\
3243\end{array}$ & $\begin{array}{l}1233 \\
1436 \\
3029 \\
3795\end{array}$ \\
\hline 2-OH-THF••OH (TS17) & $\begin{array}{l}-812 \\
261 \\
691 \\
952 \\
1130 \\
1316 \\
1443 \\
3092 \\
3793\end{array}$ & $\begin{array}{l}61 \\
315 \\
782 \\
972 \\
1181 \\
1343 \\
1479 \\
3103 \\
3890\end{array}$ & $\begin{array}{l}65 \\
422 \\
831 \\
1015 \\
1217 \\
1360 \\
1496 \\
3114\end{array}$ & $\begin{array}{l}122 \\
502 \\
869 \\
1043 \\
1248 \\
1394 \\
1878 \\
3154\end{array}$ & $\begin{array}{l}206 \\
614 \\
940 \\
1108 \\
1276 \\
1415 \\
3084 \\
3170\end{array}$ \\
\hline 2-OH-THF••OH (PC17) & $\begin{array}{l}52 \\
222 \\
510 \\
927 \\
1096 \\
1310 \\
1478 \\
3113 \\
3890\end{array}$ & $\begin{array}{l}78 \\
299 \\
523 \\
955 \\
1169 \\
1329 \\
1495 \\
3121 \\
3964\end{array}$ & $\begin{array}{l}123 \\
325 \\
708 \\
977 \\
1188 \\
1358 \\
1625 \\
3176\end{array}$ & $\begin{array}{l}137 \\
424 \\
789 \\
1007 \\
1233 \\
1421 \\
3027 \\
3234\end{array}$ & $\begin{array}{l}184 \\
444 \\
854 \\
1039 \\
1250 \\
1448 \\
3107 \\
3828\end{array}$ \\
\hline 2-OH-THF••OH (P15) & $\begin{array}{l}159 \\
528 \\
955 \\
1172 \\
1316 \\
1500 \\
3156\end{array}$ & $\begin{array}{l}187 \\
692 \\
993 \\
1208 \\
1352 \\
3048 \\
3238\end{array}$ & $\begin{array}{l}348 \\
797 \\
1012 \\
1233 \\
1413 \\
3085 \\
3897\end{array}$ & $\begin{array}{l}411 \\
853 \\
1029 \\
1244 \\
1444 \\
3096\end{array}$ & $\begin{array}{l}477 \\
920 \\
1102 \\
1302 \\
1480 \\
3112\end{array}$ \\
\hline 2-OH-THF••OH (TS18) & $\begin{array}{l}-877 \\
373 \\
717 \\
951 \\
1163 \\
1299 \\
1443 \\
3105 \\
3702\end{array}$ & $\begin{array}{l}84 \\
426 \\
822 \\
996 \\
1192 \\
1319 \\
1483 \\
3110 \\
3890\end{array}$ & $\begin{array}{l}110 \\
509 \\
859 \\
1040 \\
1229 \\
1339 \\
1504 \\
3128\end{array}$ & $\begin{array}{l}168 \\
571 \\
867 \\
1068 \\
1251 \\
1401 \\
1652 \\
3142\end{array}$ & $\begin{array}{l}223 \\
594 \\
910 \\
1082 \\
1279 \\
1426 \\
3068 \\
3161\end{array}$ \\
\hline 2-OH-THF••OH (PC18) & $\begin{array}{l}54 \\
241 \\
561\end{array}$ & $\begin{array}{l}113 \\
298 \\
602\end{array}$ & $\begin{array}{l}132 \\
386 \\
712\end{array}$ & $\begin{array}{l}186 \\
412 \\
800\end{array}$ & $\begin{array}{l}218 \\
525 \\
842\end{array}$ \\
\hline
\end{tabular}




\begin{tabular}{|c|c|c|c|c|c|}
\hline & $\begin{array}{l}921 \\
1101 \\
1310 \\
1485 \\
3098 \\
3888\end{array}$ & $\begin{array}{l}955 \\
1171 \\
1321 \\
1505 \\
3141 \\
3925\end{array}$ & $\begin{array}{l}997 \\
1210 \\
1356 \\
1652 \\
3159\end{array}$ & $\begin{array}{l}1016 \\
1237 \\
1418 \\
3058 \\
3239\end{array}$ & $\begin{array}{l}1039 \\
1252 \\
1450 \\
3086 \\
3825\end{array}$ \\
\hline $\mathrm{H}_{2} \mathrm{O}$ & 1618 & 3896 & 3997 & & \\
\hline${ }^{3} \mathrm{O}_{2}$ & 1779 & & & & \\
\hline $\mathrm{HO}_{2}$ & 1268 & 1462 & 3676 & & \\
\hline $2-\mathrm{OH}-\mathrm{THF} \bullet \bullet \mathrm{O}_{2}\left(\mathrm{RO}_{2}\right)$ & $\begin{array}{l}77 \\
407 \\
813 \\
1011 \\
1205 \\
1330 \\
1485 \\
3150\end{array}$ & $\begin{array}{l}114 \\
451 \\
853 \\
1032 \\
1242 \\
1357 \\
1504 \\
3158\end{array}$ & $\begin{array}{l}143 \\
515 \\
878 \\
1058 \\
1256 \\
1383 \\
3089 \\
3170\end{array}$ & $\begin{array}{l}300 \\
545 \\
941 \\
1122 \\
1314 \\
1409 \\
3109 \\
3887\end{array}$ & $\begin{array}{l}344 \\
699 \\
992 \\
1184 \\
1315 \\
1446 \\
3112\end{array}$ \\
\hline 2-OH-THF $\bullet \mathrm{O}_{2}(\mathrm{TS} 19)$ & $\begin{array}{l}-674 \\
305 \\
726 \\
921 \\
1198 \\
1353 \\
1636 \\
3121\end{array}$ & $\begin{array}{l}70 \\
397 \\
748 \\
995 \\
1203 \\
1375 \\
1651 \\
3136\end{array}$ & $\begin{array}{l}128 \\
432 \\
840 \\
1059 \\
1260 \\
1391 \\
1721 \\
3151\end{array}$ & $\begin{array}{l}199 \\
490 \\
850 \\
1080 \\
1322 \\
1468 \\
3084 \\
3156\end{array}$ & $\begin{array}{l}281 \\
541 \\
915 \\
1151 \\
1331 \\
1496 \\
3091\end{array}$ \\
\hline 2-OH-THF •• $\mathrm{O}_{2}(\mathrm{PC} 19)$ & $\begin{array}{l}47 \\
154 \\
601 \\
871 \\
1222 \\
1421 \\
1845 \\
3084\end{array}$ & $\begin{array}{l}70 \\
196 \\
636 \\
931 \\
1259 \\
1427 \\
1860 \\
3107\end{array}$ & $\begin{array}{l}87 \\
240 \\
653 \\
1024 \\
1313 \\
1439 \\
3041 \\
3125\end{array}$ & $\begin{array}{l}107 \\
278 \\
769 \\
1039 \\
1369 \\
1462 \\
3046 \\
3439\end{array}$ & $\begin{array}{l}131 \\
301 \\
802 \\
1120 \\
1398 \\
1563 \\
3056\end{array}$ \\
\hline Succinaldehyde & $\begin{array}{l}84 \\
599 \\
927 \\
1258 \\
1434 \\
2987\end{array}$ & $\begin{array}{l}111 \\
634 \\
1020 \\
1365 \\
1453 \\
3051\end{array}$ & $\begin{array}{l}169 \\
769 \\
1039 \\
1392 \\
1866 \\
3065\end{array}$ & $\begin{array}{l}259 \\
791 \\
1119 \\
1418 \\
1876 \\
3098\end{array}$ & $\begin{array}{l}301 \\
876 \\
1226 \\
1422 \\
2984 \\
3113\end{array}$ \\
\hline
\end{tabular}




\begin{tabular}{|l|lllll|}
\hline 2-OH-THF $\bullet \mathrm{O}_{2}(\mathrm{TS} 20)$ & -1124 & 122 & 153 & 196 & 239 \\
& 364 & 426 & 461 & 528 & 609 \\
& 646 & 756 & 816 & 874 & 921 \\
& 961 & 988 & 1013 & 1122 & 1064 \\
& 1148 & 1201 & 1216 & 1248 & 1273 \\
& 1317 & 1329 & 1399 & 1406 & 1444 \\
& 1493 & 1555 & 1686 & 3074 & 3116 \\
& 3137 & 3199 & 3252 & 3893 & \\
\hline 2-OH-THF $\bullet \mathrm{O}_{2}(\mathrm{PC} 20)$ & 52 & 61 & 118 & 159 & 178 \\
& 254 & 412 & 443 & 534 & 592 \\
& 630 & 744 & 802 & 847 & 897 \\
& 953 & 966 & 992 & 1034 & 1074 \\
& 1116 & 1169 & 1201 & 1255 & 1298 \\
& 1309 & 1331 & 1399 & 1446 & 1488 \\
& 1547 & 1700 & 3075 & 3123 & 3132 \\
& 3247 & 3273 & 3483 & 3801 & \\
\hline \multirow{2}{*}{ 2,3-dihydro-2-furanol } & 136 & 363 & 423 & 437 & 559 \\
& 736 & 785 & 846 & 894 & 950 \\
& 965 & 992 & 1033 & 1085 & 1119 \\
& 1170 & 1208 & 1260 & 1310 & 1332 \\
& 1404 & 1449 & 1486 & 1702 & 3069 \\
& 3106 & 3120 & 3237 & 3268 & 3899 \\
\hline
\end{tabular}

Table S5: Z-matrices for optimized structures of all species and complexes at the M06-2X /6-311++g(3df,3pd) level of theory.

\section{$\underline{\mathrm{HOCH}_{2}} \underline{\mathrm{CH}}_{2} \underline{\mathrm{CH}}_{2} \underline{\mathrm{CHO}}$ (4-OH-BL)}

Final structure in terms of initial Z-matrix:

$\mathrm{C}$

$\mathrm{H}, 1, \mathrm{~B} 1$

$\mathrm{C}, 1, \mathrm{~B} 2,2, \mathrm{~A} 1$

$\mathrm{H}, 3, \mathrm{~B} 3,1, \mathrm{~A} 2,2, \mathrm{D} 1,0$

$\mathrm{H}, 3, \mathrm{~B} 4,1, \mathrm{~A} 3,2, \mathrm{D} 2,0$

$\mathrm{C}, 3, \mathrm{~B} 5,1, \mathrm{~A} 4,2, \mathrm{D} 3,0$

$\mathrm{H}, 6, \mathrm{~B} 6,3, \mathrm{~A} 5,1, \mathrm{D} 4,0$

H,6,B7,3,A6,1,D5,0

C,6,B8,3,A7,1,D6,0

O,1,B9,3,A8,6,D7,0

H,10,B10,1,A9,3,D8,0

O,9,B11,6,A10,3,D9,0

H,9,B12,6,A11,3,D10,0

H,1,B13,10,A12,3,D11,0 


Variables:
B1 $=1.09135192$
B2 $=1.5240908$
B3 $=1.09137448$
B4 $=1.0901858$
B5 $=1.5232282$
B6 $=1.09418145$
B7 $=1.09451295$
B8 $=1.50457879$
B9 $=1.41020709$
B10 $=0.96253448$
$B 11=1.20058818$
$B 12=1.10545041$
B13 $=1.09437891$
A1 $=109.6525521$
A2 $=109.75107765$
A3 $=108.39106206$
A4 $=112.35380847$
A5 $=111.79903809$
A6 $=111.6195817$
A7 $=114.29703587$
A8 $=113.06501476$
A9 $=108.80084145$
A10 $=125.01139837$
A11 $=115.0551634$
A12 $=111.02826964$
D1 $=169.93497933$
D2 $=53.15928105$
$D 3=-68.67339705$
D4 $=156.31208255$
D5 $=38.17647534$
D6 $=-83.48475333$
D7 $=49.13069804$
D8 $=44.01784083$
D9 $=-2.26759992$
D10 $=179.06890865$
D11 $=-123.58766113$

\section{$\underline{\mathrm{H}_{2}} \underline{\mathrm{O}}$}

Final structure in terms of initial Z-matrix:

$\mathrm{O}$

$\mathrm{H}, 1, \mathrm{~B} 1$

H,1,B2,2, A1

Variables:

$\mathrm{B} 1=0.95871501$ 
$\mathrm{B} 2=0.95880624$

A1 $=105.05908950$

$\underline{\text { FA }}$

Final structure in terms of initial Z-matrix:

C

$\mathrm{H}, 1, \mathrm{~B} 1$

$\mathrm{O}, 1, \mathrm{~B} 2,2, \mathrm{~A} 1$

$\mathrm{O}, 1, \mathrm{~B} 3,3, \mathrm{~A} 2,2, \mathrm{D} 1,0$

H,4,B4,1,A3,3,D2,0

Variables:

B1=1.09463027

$\mathrm{B} 2=1.19107511$

B3 $=1.33582732$

B4 $=0.96704254$

A1 $=124.93395082$

$\mathrm{A} 2=124.87003115$

A3 $=107.75704424$

$\mathrm{D} 1=179.94335063$

D2 $=-0.00433616$

\section{$\underline{\mathrm{HOCH}_{2}} \underline{\mathrm{CH}}_{2} \mathrm{CH}_{2} \mathrm{CHO}$ (TSa)}

Final structure in terms of initial Z-matrix:

C

$\mathrm{H}, 1, \mathrm{~B} 1$

$\mathrm{C}, 1, \mathrm{~B} 2,2, \mathrm{~A} 1$

H,3,B3,1,A2,2,D1,0

H,3,B4,1,A3,2,D2,0

C,3,B5,1,A4,2,D3,0

H,6,B6,3,A5,1,D4,0

H,6,B7,3,A6,1,D5,0

C,6,B8,3,A7,1,D6,0

O,1,B9,3,A8,6,D7,0

H,10,B10,1,A9,3,D8,0

O,9,B11,6,A10,3,D9,0

H,1,B12,10,A11,9,D10,0

H,9,B13,6,A12,3,D11,0

Variables:

B1 $=1.08978274$

B2 $=1.5249937$

$\mathrm{B} 3=1.08844822$

B4 $=1.08710001$

B5 $=1.5295499$

B6=1.08766944 


$$
\begin{aligned}
& \mathrm{B} 7=1.09066432 \\
& \mathrm{~B} 8=1.51801189 \\
& \mathrm{~B} 9=1.43217074 \\
& \mathrm{~B} 10=1.15506266 \\
& \mathrm{~B} 11=1.30622545 \\
& \mathrm{~B} 12=1.08749094 \\
& \mathrm{~B} 13=1.0949365 \\
& \mathrm{~A} 1=111.36270828 \\
& \mathrm{~A} 2=110.39921545 \\
& \mathrm{~A} 3=111.82427211 \\
& \mathrm{~A} 4=102.93674755 \\
& \mathrm{~A} 5=112.91911417 \\
& \mathrm{~A} 6=110.92345392 \\
& \mathrm{~A} 7=104.45243626 \\
& \mathrm{~A} 8=106.31065631 \\
& \mathrm{~A} 9=113.29904443 \\
& \mathrm{~A} 10=118.72070846 \\
& \mathrm{~A} 11=108.84115336 \\
& \mathrm{~A} 12=116.10762824 \\
& \mathrm{D} 1=163.55522797 \\
& \mathrm{D} 2=42.23499307 \\
& \mathrm{D} 3=-79.46093302 \\
& \mathrm{D} 4=-162.22741658 \\
& \mathrm{D} 5=74.79276975 \\
& \mathrm{D} 6=-44.18680017 \\
& \mathrm{D} 7=36.99078907 \\
& D 8=56.27140336 \\
& \mathrm{D} 9=-67.02375028 \\
& \mathrm{D} 10=-139.15216047 \\
& \mathrm{D} 11=142.77276978
\end{aligned}
$$

\section{2-OH-THF}

Final structure in terms of initial Z-matrix:

C

$\mathrm{H}, 1, \mathrm{~B} 1$

$\mathrm{C}, 1, \mathrm{~B} 2,2, \mathrm{~A} 1$

H,3,B3,1,A2,2,D1,0

H,3,B4,1,A3,2,D2,0

$\mathrm{C}, 3, \mathrm{~B} 5,1, \mathrm{~A} 4,2, \mathrm{D} 3,0$

H,6,B6,3,A5,1,D4,0

H,6,B7,3,A6,1,D5,0

C,6,B8,3,A7,1,D6,0

O,9,B9,6,A8,3,D7,0

O,9,B10,6,A9,3,D8,0

H,9,B11,6,A10,3,D9,0

H,1,B12,10,A11,9,D10,0 


\footnotetext{
H,11,B13,9,A12,6,D11,0

Variables:

B1=1.09062829

B2 $=1.53234106$

B3 $=1.08737092$

B4 $=1.0867483$

B5 $=1.53020042$

B6 $=1.08586678$

B7 $=1.08930643$

B8 $=1.51777398$

B9 $=1.40102472$

$\mathrm{B} 10=1.40618653$

B11 $=1.09114699$

B12 $=1.08893103$

B13 $=0.96054149$

$\mathrm{A} 1=111.92302478$

A2 $=110.57020925$

A3 $=112.21980267$

A4 $=102.19618444$

A5 $=113.93321406$

A6 $=111.11479918$

$\mathrm{A} 7=100.86681801$

A8 $=105.50735154$

A9 $=107.73631437$

A10 $=114.72593646$

A11 $=109.35207671$

A12 $=107.98044253$

D1 $=146.89564043$

D2 $=25.22901231$

D3 $=-96.35274724$

D4 $=-154.92593133$

D5 $=80.79399948$

D6 $=-34.80122314$

D7 $=38.0214215$

$\mathrm{D} 8=-81.03306264$

D9 $=155.49308955$

D10=-119.12910137

D11 $=168.62424917$
}

\section{2-OH-THF (TSb)}

Final structure in terms of initial Z-matrix:

C

$\mathrm{H}, 1, \mathrm{~B} 1$

$\mathrm{C}, 1, \mathrm{~B} 2,2, \mathrm{~A} 1$

H,3,B3,1,A2,2,D1,0 


$$
\begin{aligned}
& \text { H,3,B4,1,A3,2,D2,0 } \\
& \text { C,3,B5,1,A4,2,D3,0 } \\
& \text { H,6,B6,3,A5,1,D4,0 } \\
& \text { H,6,B7,3,A6,1,D5,0 } \\
& \text { C,6,B8,3,A7,1,D6,0 } \\
& \text { O,9,B9,6,A8,3,D7,0 } \\
& \text { O,9,B10,6,A9,3,D8,0 } \\
& \text { H,9,B11,6,A10,3,D9,0 } \\
& \text { H,1,B12,10,A11,9,D10,0 } \\
& \text { H,11,B13,9,A12,6,D11,0 } \\
& \text { Variables: } \\
& \text { B1 }=1.08483244 \\
& \text { B2 }=1.52557627 \\
& \text { B3 }=1.08727544 \\
& \text { B4 }=1.09175892 \\
& \text { B5 }=1.52386064 \\
& \text { B6 }=1.36155344 \\
& \text { B7 }=1.08019784 \\
& \text { B8 }=1.42822765 \\
& \text { B9=1.31646801 } \\
& \text { B10 }=1.97654207 \\
& \text { B11 }=1.07830646 \\
& \text { B12 }=1.09064575 \\
& \text { B13 }=0.96330136 \\
& \text { A1 }=115.65251863 \\
& \text { A2 }=110.91740851 \\
& \text { A3 }=111.01608166 \\
& \text { A4 }=100.64338042 \\
& \text { A5 }=114.24018744 \\
& \text { A6 }=120.94889453 \\
& \text { A7 }=104.4346912 \\
& \text { A8 }=113.49822233 \\
& \text { A9 }=88.53824809 \\
& \text { A10 }=128.88181271 \\
& \text { A11 }=107.07693241 \\
& \text { A12 }=122.66510734 \\
& \text { D1 }=93.14781421 \\
& \text { D2 }=-27.2647132 \\
& \text { D3 }=-146.30197179 \\
& \text { D4=-60.60049341 } \\
& \text { D5 }=159.75071614 \\
& \text { D6=22.8411738 } \\
& \text { D7=-9.3499578 } \\
& \text { D8 }=-117.61360076 \\
& \text { D9 }=152.18189263 \\
& \text { D10 }=-93.8982223 \\
&
\end{aligned}
$$


D11 $=121.04902725$

\section{2-OH-THF (PC)}

Final structure in terms of initial Z-matrix:

C

$\mathrm{H}, 1, \mathrm{~B} 1$

$\mathrm{C}, 1, \mathrm{~B} 2,2, \mathrm{~A} 1$

H,3,B3,1,A2,2,D1,0

H,3,B4,1,A3,2,D2,0

C,3,B5,1,A4,2,D3,0

H,6,B6,3,A5,1,D4,0

H,6,B7,3,A6,1,D5,0

C,6,B8,3,A7,1,D6,0

O,9,B9,6,A8,3,D7,0

O,9,B10,6,A9,3,D8,0

H,9,B11,6,A10,3,D9,0

H,1,B12,10,A11,9,D10,0

H,11,B13,9,A12,6,D11,0

Variables:

B1 $=1.08696692$

$\mathrm{B} 2=1.54362778$

B3=1.08925272

B4 $=1.09010398$

B5 $=1.51144708$

$\mathrm{B} 6=2.3701677$

$\mathrm{B} 7=1.07631433$

B8 $=1.32748426$

B9 $=1.3535033$

$\mathrm{B} 10=3.34225857$

$\mathrm{B} 11=1.07933744$

B12 $=1.08685119$

$\mathrm{B} 13=0.95889221$

A1=113.18490337

A2 $=111.19585431$

A3 $=112.29095916$

A4 $=101.41427655$

A5 $=84.22870957$

A6 $=125.76038843$

A7 $=108.60546758$

A8 $=115.49797216$

A9 $=72.6327831$

A10 $=129.61730401$

A11 $=107.41368828$

A12 $=102.84787612$

D1=117.59698737 


$$
\begin{aligned}
& \mathrm{D} 2=-3.44867573 \\
& D 3=-122.63068712 \\
& D 4=-73.21308382 \\
& D 5=-178.96694001 \\
& D 6=3.63123483 \\
& D 7=-1.40718473 \\
& D 8=-71.26475696 \\
& D 9=178.29804521 \\
& D 10=-115.9851011 \\
& D 11=-163.70647264
\end{aligned}
$$

\section{2,3-DHF}

Final structure in terms of initial Z-matrix:

C

$\mathrm{H}, 1, \mathrm{~B} 1$

$\mathrm{C}, 1, \mathrm{~B} 2,2, \mathrm{~A} 1$

H,3,B3,1,A2,2,D1,0

H,3,B4,1,A3,2,D2,0

C,3,B5,1,A4,2,D3,0

H,6,B6,3,A5,1,D4,0

C,6,B7,3,A6,1,D5,0

O,8,B8,6,A7,3,D6,0

H,8,B9,6,A8,3,D7,0

H,1,B10,9,A9,8,D8,0

Variables:

B1 $=1.08588545$

$\mathrm{B} 2=1.53791038$

$\mathrm{B} 3=1.08819881$

B4 $=1.09202609$

B5 $=1.51160574$

$\mathrm{B} 6=1.0758851$

B7 $=1.32465735$

$B 8=1.36069724$

B9 $=1.07848803$

B10 $=1.09133066$

A1 $=114.23993035$

A2 $=111.46754257$

A3 $=112.34678935$

A4 $=100.56160337$

A5 $=125.89523312$

A6 $=108.21897742$

A7 $=115.11353479$

A8 $=129.96329359$

A9 $=107.4934274$

D1 $=102.84272957$

D2=-18.19489865 
D3=-136.52210536

D4 $=-171.84569235$

D5 $=12.09056135$

D6=-2.20341559

D7 $=176.58097989$

D8 $=-101.90226435$

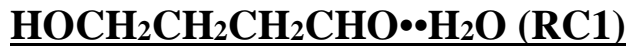

Final structure in terms of initial Z-matrix:

C

$\mathrm{H}, 1, \mathrm{~B} 1$

$\mathrm{C}, 1, \mathrm{~B} 2,2, \mathrm{~A} 1$

H,3,B3,1,A2,2,D1,0

H,3,B4,1,A3,2,D2,0

C,3,B5,1,A4,2,D3,0

H,6,B6,3,A5,1,D4,0

H,6,B7,3,A6,1,D5,0

C,6,B8,3,A7,1,D6,0

$\mathrm{O}, 1, \mathrm{~B} 9,3, \mathrm{~A} 8,6, \mathrm{D} 7,0$

H,10,B10,1,A9,3,D8,0

O,9,B11,6,A10,3,D9,0

H,9,B12,6,A11,3,D10,0

H,1,B13,10,A12,9,D11,0

O,12,B14,9,A13,6,D12,0

H,15,B15,12,A14,9,D13,0

H,15,B16,12,A15,9,D14,0

Variables:

B1 $=1.09173141$

B2 $=1.52651685$

B3 $=1.09048232$

B4=1.09052729

B5 $=1.5228424$

B6 $=1.09606681$

$\mathrm{B} 7=1.09245861$

B8 $=1.50105485$

B9 $=1.40856971$

B10 $=0.96741565$

B11 $=1.20326401$

B12 $=1.1041654$

$\mathrm{B} 13=1.09329905$

B14 $=2.78971507$

B15 $=0.9690975$

$\mathrm{B} 16=0.95837179$

A1 $=109.24385056$ 

$\mathrm{A} 2=110.15112979$
A3 $=108.72594361$
A4 $=110.97682126$
A5 $=111.55612508$
A6 $=111.45628665$
A7 $=115.15649758$
A8 $=112.50981994$
A9 $=107.32824255$
$\mathrm{A} 10=125.53891312$
A11 $=115.20880611$
A12 $=111.13398969$
A13 $=125.73327956$
A14 $=15.37248073$
$\mathrm{A} 15=114.81696076$
$\mathrm{D} 1=170.23838487$
D2 $=53.15651621$
D3 $=-67.98656746$
D4 $=156.67414341$
D5 $=38.6216539$
D6 $=-84.31863642$
$\mathrm{D} 7=49.78413854$
D8 $=72.57005152$
D9 $=-20.4073503$
D10 $=162.96850288$
D11 $=-137.14308009$
D12 $=44.65127586$
D13 $=-154.72816806$
D14=146.57044621

\section{$\underline{\mathrm{HOCH}_{2}} \underline{\mathrm{CH}_{2}} \underline{\mathrm{CH}_{2} \mathrm{CHO} \cdot \bullet \mathrm{H}_{2} \mathrm{O} \text { (TS1) }}$}

Final structure in terms of initial Z-matrix:

C

H, 1,B1

$\mathrm{C}, 1, \mathrm{~B} 2,2, \mathrm{~A} 1$

H,3,B3,1,A2,2,D1,0

H,3,B4,1,A3,2,D2,0

C,3,B5,1,A4,2,D3,0

H,6,B6,3,A5,1,D4,0

H,6,B7,3,A6,1,D5,0

C,6,B8,3,A7,1,D6,0

O,1,B9,3,A8,6,D7,0

H,10,B10,1,A9,3,D8,0

O,9,B11,6,A10,3,D9,0

H,9,B12,6,A11,3,D10,0

H,1,B13,10,A12,9,D11,0 
O,1,B14,10,A13,9,D12,0

H,15,B15,1,A14,10,D13,0

H,3,B16,1,A15,15,D14,0

Variables:

B1 $=1.08932774$

B2 $=1.52579467$

B3 $=1.0881903$

B4 $=1.087195$

B5 $=1.52667195$

B6 $=1.08670235$

B7 $=1.09066733$

B8 $=1.5178249$

B9 $=1.43717617$

$\mathrm{B} 10=1.13267501$

B11 $=1.29686495$

B12 $=1.09621433$

$\mathrm{B} 13=1.08864072$

B14=3.21033956

$\mathrm{B} 15=1.13521161$

B16 $=4.74321285$

A1 $=111.52670106$

A2 $=110.47341763$

A3 $=111.64847228$

A4 $=103.00028843$

A5 $=112.96307518$

A6 $=110.92496268$

A7 $=103.630591$

A8 $=106.28955684$

A9 $=111.83372548$

A10 $=116.19605168$

A11 $=115.34347873$

A12 $=108.91691386$

A13 $=42.96982469$

A14 $=80.1410561$

A15 $=52.25069678$

D1=156.59508906

$\mathrm{D} 2=35.12368672$

D3 $=-86.67354336$

D4 $=-164.50887005$

D5 $=72.02232718$

D6 $=-46.57689226$

D7 $=29.98417359$

D8 $=104.90745414$

D9 $=-73.80316017$

D10 $=143.60697597$

D11=-126.10685435 
D12 $=-96.44340431$

D13 $=71.67757058$

D14=6.18488956

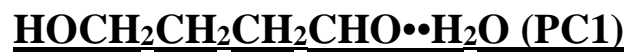

Final structure in terms of initial Z-matrix:

C

$\mathrm{H}, 1, \mathrm{~B} 1$

$\mathrm{C}, 1, \mathrm{~B} 2,2, \mathrm{~A} 1$

$\mathrm{H}, 3, \mathrm{~B} 3,1, \mathrm{~A} 2,2, \mathrm{D} 1,0$

H,3,B4,1,A3,2,D2,0

C,3,B5,1,A4,2,D3,0

H,6,B6,3,A5,1,D4,0

H,6,B7,3,A6,1,D5,0

C,6,B8,3,A7,1,D6,0

O,9,B9,6,A8,3,D7,0

H,10,B10,9,A9,6,D8,0

O,9,B11,6,A10,3,D9,0

H,9,B12,6,A11,3,D10,0

H,1,B13,10,A12,9,D11,0

O,10,B14,9,A13,6,D12,0

H,12,B15,9,A14,6,D13,0

H,15,B16,10,A15,9,D14,0

Variables:

B1 $=1.08998178$

B2 $=1.53290766$

B3 $=1.08715882$

B4 $=1.08665177$

$\mathrm{B} 5=1.53073023$

B6 $=1.0856384$

B7 $=1.08918084$

B8 $=1.51663491$

B9=1.42021937

$\mathrm{B} 10=1.91699775$

$\mathrm{B} 11=1.39198374$

B12 $=1.09142972$

$\mathrm{B} 13=1.08881472$

B14 $=2.77356089$

$\mathrm{B} 15=0.96711033$

B16 $=0.95785986$

A1 $=112.11355916$

A2 $=110.47282336$

A3 $=112.10128583$

A4 $=102.47358333$

A5 $=113.77277776$

A6 $=111.09538807$ 


$$
\begin{aligned}
& A 7=101.02719694 \\
& \text { A8 }=104.34470866 \\
& \text { A9 }=99.72166315 \\
& \text { A10 }=108.46612557 \\
& \text { A11 }=114.93693059 \\
& \text { A12 }=109.42841102 \\
& \text { A13 }=88.85544326 \\
& \text { A14 }=107.95331516 \\
& \text { A15 }=119.44408032 \\
& \text { D1 }=145.33851742 \\
& \text { D2 }=23.81698349 \\
& \text { D3 }=-97.82386404 \\
& D 4=-154.99515691 \\
& \text { D5 }=80.73681927 \\
& \text { D6 }=-35.19556892 \\
& D 7=39.71438774 \\
& \text { D8 }=-167.48531217 \\
& \text { D9 }=-79.41846424 \\
& \text { D10 }=155.32030198 \\
& \text { D11 }=-115.96398847 \\
& \text { D12 }=-164.49951405 \\
& \text { D13 }=173.91668548 \\
& \text { D14 }=-104.69609244
\end{aligned}
$$

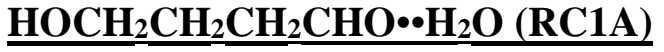

Final structure in terms of initial Z-matrix:

C

$\mathrm{H}, 1, \mathrm{~B} 1$

$\mathrm{C}, 1, \mathrm{~B} 2,2, \mathrm{~A} 1$

H,3,B3,1,A2,2,D1,0

H,3,B4,1,A3,2,D2,0

C,3,B5,1,A4,2,D3,0

H,6,B6,3,A5,1,D4,0

H,6,B7,3,A6,1,D5,0

C,6,B8,3,A7,1,D6,0

O,1,B9,3,A8,6,D7,0

$\mathrm{H}, 10, \mathrm{~B} 10,1, \mathrm{~A} 9,3, \mathrm{D} 8,0$

O,9,B11,6,A10,3,D9,0

O,12,B12,9,A11,6,D10,0

H,13,B13,12,A12,9,D11,0

H,13,B14,12,A13,9,D12,0

H,9,B15,6,A14,3,D13,0

H,1,B16,10,A15,9,D14,0

Variables:

B1 $=1.09174367$

B2 $=1.52652579$ 


$$
\begin{aligned}
& \text { B3 }=1.09048655 \\
& \text { B4 }=1.09053225 \\
& \text { B5 }=1.52280389 \\
& \text { B6 }=1.09610706 \\
& \text { B7 }=1.09244324 \\
& \text { B8 }=1.50103533 \\
& \text { B9 }=1.40854976 \\
& \text { B10 }=0.9675029 \\
& \text { B11 }=1.20325955 \\
& \text { B12 }=2.78938474 \\
& \text { B13 }=0.95835881 \\
& \text { B14 }=0.96913762 \\
& \text { B15 }=1.10415643 \\
& \text { B16 }=1.09329502 \\
& \text { A1 }=109.23837228 \\
& \text { A2 }=110.15848975 \\
& \text { A3 }=108.72496559 \\
& \text { A4 }=110.97569851 \\
& \text { A5 }=111.54835518 \\
& \text { A6 }=111.46012205 \\
& \text { A7 }=115.16792864 \\
& \text { A8 }=112.51164361 \\
& \text { A9 }=107.33611332 \\
& \text { A10 }=125.53975055 \\
& \text { A11 }=125.65093244 \\
& \text { A12 }=114.75701085 \\
& \text { A13 }=15.29780323 \\
& \text { A14 }=115.20893859 \\
& \text { A15 }=111.13549033 \\
& \text { D1 }=170.23127686 \\
& \text { D2 }=53.15173634 \\
& \text { D3 }=-67.98523169 \\
& \text { D4 }=156.73393934 \\
& \text { D5 }=38.69118443 \\
& \text { D6 }=-84.28025863 \\
& \text { D7 }=49.78717557 \\
& \text { D8 }=72.66789165 \\
& \text { D9 }=-20.48184574 \\
& \text { D10 }=44.72485659 \\
& \text { D11 }=146.96422356 \\
& \text { D12 }=-154.25920544 \\
& \text { D13 }=162.92326209 \\
& \text { D14 }=-137.16093413
\end{aligned}
$$

\section{$\underline{\mathrm{HOCH}_{2} \mathrm{CH}_{2} \mathrm{CH}_{2} \mathrm{CHO} \cdot \bullet \mathrm{H}_{2} \mathrm{O} \text { (TS1A) }}$}


Final structure in terms of initial Z-matrix:

C

$\mathrm{H}, 1, \mathrm{~B} 1$

$\mathrm{C}, 1, \mathrm{~B} 2,2, \mathrm{~A} 1$

$\mathrm{H}, 3, \mathrm{~B} 3,1, \mathrm{~A} 2,2, \mathrm{D} 1,0$

H,3,B4,1,A3,2,D2,0

C,3,B5,1,A4,2,D3,0

H,6,B6,3,A5,1,D4,0

H,6,B7,3,A6,1,D5,0

C,6,B8,3,A7,1,D6,0

O,1,B9,3,A8,6,D7,0

H,10,B10,1,A9,3,D8,0

O,9,B11,6,A10,3,D9,0

O,12,B12,9,A11,6,D10,0

H,13,B13,12,A12,9,D11,0

H,13,B14,12,A13,9,D12,0

H,9,B15,6,A14,3,D13,0

H,1,B16,10,A15,9,D14,0

Variables:

B1=1.08932425

$\mathrm{B} 2=1.52504336$

$\mathrm{B} 3=1.08818317$

B4 $=1.0872821$

$\mathrm{B} 5=1.52690502$

$\mathrm{B} 6=1.08673022$

B7 $=1.09066739$

$\mathrm{B} 8=1.51809537$

B9=1.4371809

$\mathrm{B} 10=1.13315476$

$\mathrm{B} 11=1.29700831$

$\mathrm{B} 12=2.36997359$

B13 $=0.95892082$

B14=1.13585901

$\mathrm{B} 15=1.09623071$

B16=1.08866302

A1=111.55191709

A2 $=110.46496037$

A3 $=111.66425681$

A4 $=102.99278535$

A5 $=112.97969074$

A6 $=110.92046404$

A7 $=103.66134933$

A8 $=106.28528044$

A9 $=111.83652443$

$\mathrm{A} 10=116.19616191$

A11 $=98.52476492$ 

A12 $=118.15167236$
A13 $=12.54116641$
A14 $=115.32056462$
A15 $=108.89783216$
D1 $=156.84263947$
$\mathrm{D} 2=35.33160655$
D3 $=-86.41600144$
D4 $=-164.52509981$
D5 $=72.03663703$
D6 $=-46.58452476$
D7 $=30.25222664$
D8 $=104.51743439$
D9 $=-74.0749966$
D10 $=124.44178419$
D11 $=98.62881422$
D12 $=155.91254268$
D13 $=143.40343125$
D14=-126.52893796

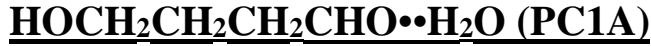

Final structure in terms of initial Z-matrix:

$\mathrm{C}$

$\mathrm{H}, 1, \mathrm{~B} 1$

$\mathrm{C}, 1, \mathrm{~B} 2,2, \mathrm{~A} 1$

H,3,B3,1,A2,2,D1,0

H,3,B4,1,A3,2,D2,0

C,3,B5,1,A4,2,D3,0

H,6,B6,3,A5,1,D4,0

H,6,B7,3,A6,1,D5,0

C,6,B8,3,A7,1,D6,0

O,9,B9,6,A8,3,D7,0

H,10,B10,9, A9,6,D8,0

O,9,B11,6,A10,3,D9,0

O,10,B12,9,A11,6,D10,0

H,13,B13,10,A12,9,D11,0

H,12,B14,9,A13,6,D12,0

H,9,B15,6,A14,3,D13,0

H,1,B16,10,A15,9,D14,0

Variables:

B1 $=1.09000804$

$\mathrm{B} 2=1.53279966$

B3 $=1.0871684$

B4 $=1.0866555$

B5 $=1.53070228$

B6 $=1.08563266$

B7 $=1.08919242$ 


$$
\begin{aligned}
& B 8=1.51669371 \\
& B 9=1.42016982 \\
& B 10=1.91966834 \\
& B 11=1.39197638 \\
& B 12=2.77494174 \\
& B 13=0.95787113 \\
& B 14=0.96713997 \\
& B 15=1.0914404 \\
& B 16=1.08880364 \\
& A 1=112.10055817 \\
& A 2=110.47587264 \\
& A 3=112.10129267 \\
& A 4=102.45910236 \\
& A 5=113.77912133 \\
& A 6=111.09126901 \\
& A 7=101.01924884 \\
& A 8=104.35259706 \\
& A 9=99.72898404 \\
& A 10=108.46694963 \\
& A 11=88.80179441 \\
& A 12=119.36593519 \\
& A 13=107.93880658 \\
& A 14=114.92846111 \\
& A 15=109.42647831 \\
& D 1=145.48733124 \\
& D 2=23.95821544 \\
& D 3=-97.68546321 \\
& D 4=-155.06322827 \\
& D 5=80.66312718 \\
& D 6=-35.26683494 \\
& D 7=39.6993895 \\
& D 8=-167.41876394 \\
& D 9=-79.43752599 \\
& D 10=-164.4777823 \\
& D 11=-104.52747218 \\
& D 12=173.93266785 \\
& D 13=155.31176039 \\
& D 14=-116.1276845
\end{aligned}
$$

\section{2-OH-THF•• $\mathrm{H}_{2} \mathrm{O}(\mathrm{RC} 2)$}

Final structure in terms of initial Z-matrix:
$\mathrm{C}$
$\mathrm{H}, 1, \mathrm{~B} 1$
$\mathrm{C}, 1, \mathrm{~B} 2,2, \mathrm{~A} 1$
H,3,B3,1,A2,2,D1,0
H,3,B4,1,A3,2,D2,0 

C,3,B5,1,A4,2,D3,0
H,6,B6,3,A5,1,D4,0
H,6,B7,3,A6,1,D5,0
C,6,B8,3,A7,1,D6,0
O,9,B9,6,A8,3,D7,0
O,9,B10,6,A9,3,D8,0
H,9,B11,6,A10,3,D9,0
H,1,B12,10,A11,9,D10,0
H,11,B13,9,A12,6,D11,0
O,11,B14,9,A13,6,D12,0
H,15,B15,11,A14,9,D13,0
H,15,B16,11,A15,9,D14,0
Variables:
B1 $=1.08612125$
$\mathrm{B} 2=1.53148958$
B3 $=1.08572588$
B4 $=1.08746074$
B5 $=1.54352537$
B6 $=1.08655311$
$\mathrm{B} 7=1.08681245$
$\mathrm{B} 8=1.52858531$
B9 $=1.39081032$
$\mathrm{B} 10=1.42178512$
$\mathrm{B} 11=1.08954426$
B12 $=1.0914831$
$\mathrm{B} 13=0.96171248$
$\mathrm{B} 14=2.84736261$
B15 $=0.96782929$
B16 $=0.95797064$
A1 $=114.10935064$
A2 $=111.14749455$
A3 $=110.61194527$
A4 $=103.43405593$
A5 $=112.63521791$
A6 $=111.51516724$
A7 $=103.85665993$
A8 $=106.66684781$
A9 $=108.01839009$
A10 $=114.76687111$
A11 $=109.92122795$
A12 $=107.60149156$
A13 $=107.6241846$
A14 $=9.40088982$
A15 $=114.38421841$
D1 $=98.27440723$
D2 $=-23.12356434$ 


$$
\begin{aligned}
& \mathrm{D} 3=-141.60352084 \\
& \mathrm{D} 4=-115.87432803 \\
& \mathrm{D} 5=121.75698694 \\
& \mathrm{D} 6=3.48299703 \\
& \mathrm{D} 7=19.07281176 \\
& \mathrm{D} 8=-100.01189854 \\
& \mathrm{D} 9=137.80600161 \\
& \mathrm{D} 10=-81.143246 \\
& \mathrm{D} 11=168.98690087 \\
& \mathrm{D} 12=35.88822257 \\
& \mathrm{D} 13=-174.71979011 \\
& \mathrm{D} 14=-152.21763817
\end{aligned}
$$

\section{$\underline{2-\mathrm{OH}-\mathrm{THF} \bullet \bullet \mathrm{H}_{2} \mathrm{O} \text { (TS2) }}$}

Final structure in terms of initial Z-matrix:

C

$\mathrm{H}, 1, \mathrm{~B} 1$

$\mathrm{C}, 1, \mathrm{~B} 2,2, \mathrm{~A} 1$

H,3,B3,1,A2,2,D1,0

H,3,B4,1,A3,2,D2,0

C,3,B5,1,A4,2,D3,0

H,6,B6,3,A5,1,D4,0

H,6,B7,3,A6,1,D5,0

$\mathrm{C}, 6, \mathrm{~B} 8,3, \mathrm{~A} 7,1, \mathrm{D} 6,0$

O,9,B9,6,A8,3,D7,0

O,9,B10,6,A9,3,D8,0

H,9,B11,6,A10,3,D9,0

H,1,B12,10,A11,9,D10,0

H,11,B13,9,A12,6,D11,0

O,11,B14,9,A13,6,D12,0

H,11,B15,9,A14,6,D13,0

H,15,B16,11,A15,9,D14,0

Variables:

B1 $=1.08457508$

B2 $=1.52200752$

B3 $=1.08751059$

B4=1.09103259

$\mathrm{B} 5=1.53238187$

$\mathrm{B} 6=1.31311168$

B7 $=1.08290805$

$B 8=1.42971081$

B9=1.32136738

$\mathrm{B} 10=1.98099629$

$\mathrm{B} 11=1.0779422$

$\mathrm{B} 12=1.09101019$

$\mathrm{B} 13=0.96092907$ 


$$
\begin{aligned}
& \mathrm{B} 14=2.39486461 \\
& \mathrm{~B} 15=1.09166014 \\
& \mathrm{~B} 16=0.95900074 \\
& \mathrm{~A} 1=115.86359378 \\
& \mathrm{~A} 2=110.95890885 \\
& \mathrm{~A} 3=111.03205178 \\
& \mathrm{~A} 4=100.36670954 \\
& \mathrm{~A} 5=104.06684267 \\
& \mathrm{~A} 6=119.18551972 \\
& \mathrm{~A} 7=103.8163025 \\
& \mathrm{~A} 8=113.3758782 \\
& \mathrm{~A} 9=105.7496642 \\
& \mathrm{~A} 10=126.60821769 \\
& \mathrm{~A} 11=107.55634487 \\
& \mathrm{~A} 12=106.19025074 \\
& \mathrm{~A} 13=88.99867143 \\
& \mathrm{~A} 14=99.30484633 \\
& \mathrm{~A} 15=119.07650713 \\
& \mathrm{D} 1=91.10596428 \\
& \mathrm{D} 2=-30.21467617 \\
& \mathrm{D} 3=-149.40469823 \\
& \mathrm{D} 4=-83.4082478 \\
& \mathrm{D} 5=154.59209604 \\
& \mathrm{D} 6=24.6054288 \\
& \mathrm{D} 7=-8.40227302 \\
& \mathrm{D} 8=-118.01551504 \\
& \mathrm{D} 9=144.4569073 \\
& \mathrm{D} 10=-88.99516044 \\
& \mathrm{D} 11=129.43247975 \\
& \mathrm{D} 12=15.50266074 \\
& \mathrm{D} 13=16.16314542 \\
& \mathrm{D} 14=-122.13184087
\end{aligned}
$$

\section{2-OH-THF••H2O (PC2)}

Final structure in terms of initial Z-matrix:

C

$\mathrm{H}, 1, \mathrm{~B} 1$

$\mathrm{C}, 1, \mathrm{~B} 2,2, \mathrm{~A} 1$

H,3,B3,1,A2,2,D1,0

H,3,B4,1,A3,2,D2,0

C,3,B5,1,A4,2,D3,0

H,6,B6,3,A5,1,D4,0

H,6,B7,3,A6,1,D5,0

C,6,B8,3,A7,1,D6,0

O,9,B9,6,A8,3,D7,0

O,9,B10,6,A9,3,D8,0 

H,9,B11,6,A10,3,D9,0
H,1,B12,10,A11,9,D10,0
H,11,B13,9,A12,6,D11,0
O,11,B14,9,A13,6,D12,0
H,11,B15,9,A14,6,D13,0
H,15,B16,11,A15,9,D14,0
Variables:
B1 $=1.0854745$
B2 $=1.53946972$
B3 $=1.08831613$
B4 $=1.09098861$
$\mathrm{B} 5=1.51199502$
B6 $=2.32195909$
$\mathrm{B} 7=1.07690069$
B8 $=1.32758891$
B9 $=1.35734159$
B10 $=3.50625328$
B11 $=1.07909987$
B12 $=1.08992852$
$\mathrm{B} 13=0.9613853$
B14 $=2.95058927$
B15 $=0.96436675$
$\mathrm{B} 16=0.95945831$
A1 $=114.09180408$
A2 $=111.10797667$
A3 $=112.44393915$
A4 $=101.04649387$
A5 $=88.84434724$
A6 $=125.88842051$
$\mathrm{A} 7=108.49985944$
A8 $=115.08034482$
A9 $=119.65128259$
A10 $=129.93061108$
A11 $=107.3379834$
A12 $=38.70306151$
A13 $=61.73497724$
A14 $=72.17809441$
A15 $=113.43480567$
D1 $=107.48300903$
D2 $=-13.84860994$
D3 $=-132.29006831$
D4 $=-74.50028738$
D5 $=-175.32567675$
D6 $=9.8109459$
D7 $=-1.92709407$
D8 $=-65.07105011$ 


$$
\begin{aligned}
& D 9=176.98755292 \\
& D 10=-105.73730131 \\
& D 11=131.16755381 \\
& D 12=-14.02305401 \\
& \text { D13 }=-12.56756838 \\
& D 14=-129.12222269
\end{aligned}
$$

\section{$\underline{\mathrm{HOCH}} 2 \mathrm{CH}_{2} \mathrm{CH}_{2}{ }_{\mathrm{CHO} \cdot \mathrm{HO}_{2}}$ (TS3)}

Final structure in terms of initial Z-matrix:

C

$\mathrm{H}, 1, \mathrm{~B} 1$

$\mathrm{C}, 1, \mathrm{~B} 2,2, \mathrm{~A} 1$

H,3,B3,1,A2,2,D1,0

H,3,B4,1,A3,2,D2,0

C,3,B5,1,A4,2,D3,0

H,6,B6,3,A5,1,D4,0

H,6,B7,3,A6,1,D5,0

$\mathrm{C}, 6, \mathrm{~B} 8,3, \mathrm{~A} 7,1, \mathrm{D} 6,0$

O,1,B9,3,A8,6,D7,0

H,10,B10,1,A9,3,D8,0

O,9,B11,6,A10,3,D9,0

H,9,B12,6,A11,3,D10,0

H,1,B13,10,A12,9,D11,0

O,12,B14,9,A13,6,D12,0

H,12,B15,9,A14,6,D13,0

O,15,B16,12,A15,9,D14,0

Variables:

B1=1.08688142

$\mathrm{B} 2=1.52812269$

$\mathrm{B} 3=1.08680164$

B4 $=1.09052568$

B5 $=1.52041642$

B6 $=1.09140815$

B7 $=1.08933829$

$\mathrm{B} 8=1.51220116$

$\mathrm{B} 9=1.43336034$

B10 $=1.047444$

$\mathrm{B} 11=1.27447597$

B12 $=1.09216758$

B13 $=1.09051592$

B14 $=2.39190356$

B15=1.18616809

B16=1.29790956

A1=113.22319119

A2 $=111.61910246$ 


$$
\begin{aligned}
& \text { A3 }=110.1522147 \\
& \text { A }=103.87171193 \\
& \text { A5 }=111.69535675 \\
& \text { A6 }=112.75540826 \\
& \text { A7 }=106.38515684 \\
& \text { A8 }=105.84503864 \\
& \text { A9 }=107.67120879 \\
& \text { A10 }=121.91608124 \\
& \text { A11 }=114.40004491 \\
& \text { A12 }=108.4637044 \\
& \text { A13 }=109.48507794 \\
& \text { A14 }=112.68897486 \\
& \text { A15 }=98.5681105 \\
& \text { D1 }=84.60443806 \\
& \text { D2 }=-35.42938236 \\
& \text { D3 }=-153.34027244 \\
& \text { D4 }=-70.59510392 \\
& \text { D5 }=166.7607207 \\
& \text { D6 }=47.98345315 \\
& \text { D7 }=-34.2918264 \\
& \text { D8 }=116.26971485 \\
& \text { D9 }=-150.70012974 \\
& \text { D10 }=62.66778818 \\
& \text { D11 }=-108.67664177 \\
& \text { D12 }=56.74660617 \\
& \text { D13 }=50.76335982 \\
& \text { D14 }=48.18374699
\end{aligned}
$$

\section{$\underline{\mathrm{HOCH}_{2} \mathrm{CH}_{2} \mathrm{CH}_{2} \mathrm{CHO} \cdot \cdot \mathrm{HO}_{2}(\mathrm{RC} 3)}$}

Final structure in terms of initial Z-matrix:

C

$\mathrm{H}, 1, \mathrm{~B} 1$

$\mathrm{C}, 1, \mathrm{~B} 2,2, \mathrm{~A} 1$

H,3,B3,1,A2,2,D1,0

H,3,B4,1,A3,2,D2,0

C,3,B5,1,A4,2,D3,0

H,6,B6,3,A5,1,D4,0

H,6,B7,3,A6,1,D5,0

C,6,B8,3,A7,1,D6,0

O,1,B9,3,A8,6,D7,0

H,10,B10,1,A9,3,D8,0

O,9,B11,6,A10,3,D9,0

H,9,B12,6,A11,3,D10,0

H,1,B13,10,A12,3,D11,0

O,12,B14,9,A13,6,D12,0 
H,15,B15,12,A14,9,D13,0

O,15,B16,12,A15,9,D14,0

Variables:

B1 $=1.09045375$

B2 $=1.52195066$

B3 $=1.08979352$

B4 $=1.09236082$

$\mathrm{B} 5=1.52788475$

B6 $=1.0907091$

B7 $=1.09489238$

$\mathrm{B} 8=1.49800791$

B9 $=1.4139501$

B10 $=0.96489347$

B11 $=1.21018153$

$\mathrm{B} 12=1.10091514$

B13 $=1.0929311$

B14 $=2.60740572$

$\mathrm{B} 15=1.00183178$

$\mathrm{B} 16=1.29993615$

A1 $=110.23959982$

A2 $=110.2551788$

A3 $=109.02821766$

A4 $=110.61881005$

A5 $=111.81003614$

A6 $=109.56086072$

$\mathrm{A} 7=112.2976404$

A8 $=109.7376563$

A9 $=107.99199546$

$\mathrm{A} 10=124.88167652$

A11 $=115.87189679$

A12 $=110.4514937$

A13 $=115.40461732$

A14 $=9.38853317$

A15 $=95.56988291$

D1 $=62.28993345$

D2 $=-55.38455844$

D3 $=-175.91305163$

D4 $=-55.9511587$

D5 $=-175.57531275$

D6 $=67.66124876$

D7 $=-56.49734418$

D8 $=105.363771$

D9 $=-135.04207486$

D10 $=48.40380044$

D11 $=-121.33065876$

D12 $=29.66616829$ 
D13 $=-131.73107432$

D14 $=50.79469438$

\section{$\underline{\mathrm{HOCH}_{2} \mathrm{CH}_{2} \mathrm{CH}_{2} \mathrm{CHO} \cdot \mathrm{HO}_{2}(\mathrm{PC} 3)}$}

Final structure in terms of initial Z-matrix:

C

$\mathrm{H}, 1, \mathrm{~B} 1$

$\mathrm{C}, 1, \mathrm{~B} 2,2, \mathrm{~A} 1$

H,3,B3,1,A2,2,D1,0

H,3,B4,1,A3,2,D2,0

C,3,B5,1,A4,2,D3,0

H,6,B6,3,A5,1,D4,0

H,6,B7,3,A6,1,D5,0

C,6,B8,3,A7,1,D6,0

O,1,B9,3,A8,6,D7,0

H,10,B10,1,A9,3,D8,0

O,9,B11,6,A10,3,D9,0

H,9,B12,6,A11,3,D10,0

H,1,B13,10,A12,9,D11,0

O,12,B14,9,A13,6,D12,0

H,12,B15,9,A14,6,D13,0

O,15,B16,12,A15,9,D14,0

Variables:

B1 $=1.08747442$

B2 $=1.52461602$

B3 $=1.08668513$

B4 $=1.08994731$

B5 $=1.52285367$

B6 $=1.09211964$

B7 $=1.08629591$

B8 $=1.52398857$

B9 $=1.43338303$

B10 $=1.65283356$

B11 $=1.37537382$

B12 $=1.09005798$

B13 $=1.09295044$

B14 $=2.86678318$

B15 $=0.96808352$

$\mathrm{B} 16=1.30052317$

A1 $=113.85995542$

A2 $=112.37720692$

A3 $=110.31373557$

A4 $=101.16774107$

A5 $=110.56263392$

A6=114.47366936 


$$
\begin{aligned}
& A 7=102.74795814 \\
& A 8=105.33372218 \\
& A 9=107.27903623 \\
& A 10=115.87145876 \\
& A 11=112.35052255 \\
& A 12=108.92264817 \\
& A 13=96.51087064 \\
& \text { A14 }=108.46296873 \\
& \text { A15 }=88.04429572 \\
& D 1=86.62360564 \\
& \text { D2 }=-34.60204835 \\
& \text { D3 }=-151.70408528 \\
& \text { D4 }=-76.66692057 \\
& \text { D5 }=160.01390442 \\
& \text { D6 }=38.72474408 \\
& \text { D7 }=-33.16045726 \\
& \text { D8 }=135.32861921 \\
& \text { D9 }=-152.21228836 \\
& \text { D10 }=85.25092088 \\
& \text { D11 }=-104.53963351 \\
& \text { D12 }=75.33946114 \\
& \text { D13 }=53.2269866 \\
& \text { D14 }=73.12834265
\end{aligned}
$$

\section{$\underline{2-\mathrm{OH}-\mathrm{THF} \cdot \bullet \mathrm{HO}_{2} \text { (RC4) }}$}

Final structure in terms of initial Z-matrix:

C

$\mathrm{H}, 1, \mathrm{~B} 1$

$\mathrm{C}, 1, \mathrm{~B} 2,2, \mathrm{~A} 1$

H,3,B3,1,A2,2,D1,0

H,3,B4,1,A3,2,D2,0

C,3,B5,1,A4,2,D3,0

H,6,B6,3,A5,1,D4,0

H,6,B7,3,A6,1,D5,0

C,6,B8,3,A7,1,D6,0

O,9,B9,6,A8,3,D7,0

O,9,B10,6,A9,3,D8,0

H,9,B11,6,A10,3,D9,0

H,1,B12,10,A11,9,D10,0

H,11,B13,9,A12,6,D11,0

O,11,B14,9,A13,6,D12,0

H,15,B15,11,A14,9,D13,0

O,15,B16,11,A15,9,D14,0

Variables:

B1=1.08595786 


B2 $=1.52516468$
B3 $=1.08578906$
B4 $=1.08814754$
B5 $=1.53977678$
B6 $=1.0868465$
B7 $=1.08648997$
B8 $=1.53221001$
B9 $=1.38821473$
B10 $=1.42876055$
B11 $=1.08897092$
B12 $=1.09145439$
B13 $=0.96209116$
B14 $=2.68674896$
B15 $=0.99027742$
B16 $=1.30360214$
A1 $=114.35931001$
A2 $=111.9839434$
A3 $=110.00336429$
A4 $=102.84814514$
A5 $=112.13735557$
A6 $=112.05699897$
A7 $=103.81331574$
A8 $=107.33966545$
A9 $=108.33300599$
A10 $=114.78739178$
A11 $=109.98470359$
A12 $=107.75605261$
A13 $=121.87507685$
A14 $=4.1353066$
A15 $=100.82923234$
D1 $=90.89128355$
D2 $=-30.62538572$
D3 $=-148.35475619$
D4 $=-105.6071184$
D5 $=132.42505414$
D6 $=13.06504808$
D7 $=9.95929653$
D8 $=-109.33352799$
D9 $=129.43458651$
D10 $=-79.2998729$
D11 $=170.08506727$
D12 $=28.15162881$
D13 $=-164.86750359$
D14 $=17.53572385$

$\underline{\text { 2-OH-THF } \bullet \mathrm{HO}_{2} \text { (TS4) }}$ 
Final structure in terms of initial Z-matrix:

C

$\mathrm{H}, 1, \mathrm{~B} 1$

$\mathrm{C}, 1, \mathrm{~B} 2,2, \mathrm{~A} 1$

H,3,B3,1,A2,2,D1,0

H,3,B4,1,A3,2,D2,0

$\mathrm{C}, 3, \mathrm{~B} 5,1, \mathrm{~A} 4,2, \mathrm{D} 3,0$

H,6,B6,3,A5,1,D4,0

H,6,B7,3,A6,1,D5,0

$\mathrm{C}, 6, \mathrm{~B} 8,3, \mathrm{~A} 7,1, \mathrm{D} 6,0$

O,9,B9,6,A8,3,D7,0

O,9,B10,6,A9,3,D8,0

H,9,B11,6,A10,3,D9,0

H,1,B12,10,A11,9,D10,0

H,11,B13,9,A12,6,D11,0

O,11,B14,9,A13,6,D12,0

H,11,B15,9,A14,6,D13,0

O,15,B16,11,A15,9,D14,0

Variables:

B1 $=1.08337233$

B2 $=1.52040509$

B3 $=1.08590775$

B4 $=1.08905567$

$\mathrm{B} 5=1.53764603$

$\mathrm{B} 6=1.1671579$

B7 $=1.08501373$

B8 $=1.43707103$

$\mathrm{B} 9=1.27934967$

$\mathrm{B} 10=2.29261859$

$\mathrm{B} 11=1.07827546$

$\mathrm{B} 12=1.08973875$

$\mathrm{B} 13=0.95918137$

$\mathrm{B} 14=2.53678791$

$\mathrm{B} 15=1.0212381$

$\mathrm{B} 16=1.29674284$

A1 $=116.44626033$

A2 $=111.1111545$

A3 $=111.47104851$

A4=100.39915454

A5 $=103.91858838$

A6 $=118.74824923$

A7 $=102.58209726$

A8 $=114.64068387$

A9 $=99.01897281$

A10 $=128.22853554$ 

$\mathrm{A} 11=105.80588711$
A12 $=124.03084666$
A13 $=95.87210896$
A14 $=101.18172954$
A15 $=98.23697796$
$\mathrm{D} 1=93.95337635$
D2 $=-29.12919268$
D3=-148.28116391
D4=-86.51155535
D5 $=153.79240581$
D6 $=26.70628092$
$\mathrm{D} 7=-12.42831857$
D8 $=-117.44541494$
D9 $=158.09417033$
D10 $=-91.19342077$
D11 $=163.88075166$
D12 $=40.76941758$
D13 $=44.46651959$
D14=-56.18465377

\section{$\underline{2-\mathrm{OH}-\mathrm{THF} \cdot \bullet \mathrm{HO}_{2}}$ (PC4)}

Final structure in terms of initial Z-matrix:

C

H,1,B1

$\mathrm{C}, 1, \mathrm{~B} 2,2, \mathrm{~A} 1$

H,3,B3,1,A2,2,D1,0

H,3,B4,1,A3,2,D2,0

C,3,B5,1,A4,2,D3,0

H,6,B6,3,A5,1,D4,0

H,6,B7,3,A6,1,D5,0

$\mathrm{C}, 6, \mathrm{~B} 8,3, \mathrm{~A} 7,1, \mathrm{D} 6,0$

O,9,B9,6,A8,3,D7,0

O,10,B10,9,A9,6,D8,0

H,9,B11,6,A10,3,D9,0

H,1,B12,10,A11,9,D10,0

H,11,B13,10,A12,9,D11,0

O,11,B14,10,A13,9,D12,0

H,11,B15,10,A14,9,D13,0

O,15,B16,11,A15,10,D14,0

Variables:

B1=1.0898832

B2 $=1.53669439$

B3=1.09034225

B4 $=1.08818095$

B5 $=1.50904357$ 


$$
\begin{aligned}
& \text { B6 }=3.55540525 \\
& \text { B7 }=1.0761309 \\
& \text { B8 }=1.32102206 \\
& \text { B9 }=1.37467704 \\
& \text { B10 }=2.74357103 \\
& \text { B11 }=1.07838984 \\
& \text { B12 }=1.08539235 \\
& \text { B13 }=0.97370084 \\
& \text { B14 }=3.01145206 \\
& \text { B15 }=0.95943526 \\
& \text { B16 }=1.30327249 \\
& \text { A1 }=111.60364984 \\
& \text { A2 }=112.00578068 \\
& \text { A3 }=111.38106391 \\
& \text { A4 }=101.09153488 \\
& \text { A5 }=81.39089576 \\
& \text { A6 }=125.6300701 \\
& \text { A7 }=108.95185734 \\
& \text { A8 }=114.54374848 \\
& \text { A9 }=96.95410814 \\
& \text { A10 }=130.67141096 \\
& \text { A11 }=107.82021706 \\
& \text { A12 }=16.09376487 \\
& \text { A13 }=72.37203303 \\
& \text { A14 }=120.67837173 \\
& \text { A15 }=60.80169387 \\
& \text { D1 }=140.98914534 \\
& \text { D2 }=19.55785958 \\
& \text { D3 }=-100.79101021 \\
& \text { D4 }=-68.69113359 \\
& \text { D5 }=172.65555451 \\
& \text { D6 }=-10.59813984 \\
& \text { D7 }=1.5765767 \\
& \text { D8 }=-116.79415223 \\
& \text { D9 }=-176.83125588 \\
& \text { D10 }=-137.47314855 \\
& \text { D11 }=-152.60962586 \\
& \text { D12 }=97.75266282 \\
& \text { D13 }=178.46278114 \\
& \text { D14 }=-101.30704958 \\
&
\end{aligned}
$$

\section{$\underline{\mathrm{HOCH}}_{2} \underline{\mathrm{CH}}_{2} \mathrm{CH}_{2}{ }_{\mathrm{CHO}}^{\mathrm{CHO}} \mathrm{HO}_{2}$ (RC3A)}

Final structure in terms of initial Z-matrix:

C

H,1,B1 


\section{$\mathrm{C}, 1, \mathrm{~B} 2,2, \mathrm{~A} 1$}

H,3,B3,1,A2,2,D1,0

H,3,B4,1,A3,2,D2,0

C,3,B5,1,A4,2,D3,0

H,6,B6,3,A5,1,D4,0

H,6,B7,3,A6,1,D5,0

C,6,B8,3,A7,1,D6,0

O,1,B9,3,A8,6,D7,0

H,10,B10,1,A9,3,D8,0

O,9,B11,6,A10,3,D9,0

H,9,B12,6,A11,3,D10,0

H,1,B13,10,A12,3,D11,0

O,12,B14,9,A13,6,D12,0

H,15,B15,12,A14,9,D13,0

O,15,B16,12,A15,9,D14,0

Variables:

B1 $=1.09075656$

$\mathrm{B} 2=1.52391003$

B3 $=1.09086454$

B4 $=1.08992274$

$\mathrm{B} 5=1.53295005$

B6 $=1.09161973$

B7 $=1.08948759$

$\mathrm{B} 8=1.50314175$

$\mathrm{B} 9=1.41214614$

B10 $=0.96874384$

B11 $=1.2103391$

B12 $=1.09886501$

B13 $=1.0926506$

$\mathrm{B} 14=2.639211$

$\mathrm{B} 15=0.99088953$

$\mathrm{B} 16=1.29884791$

A1 $=109.66160857$

A2 $=110.42747247$

A3 $=109.84725213$

A4 $=109.83447522$

A5 $=110.17623091$

A6 $=111.02198679$

A7 $=109.77252461$

A8 $=111.5480725$

A9 $=110.00649762$

A10 $=121.51960322$

A11 $=118.25465081$

A12 $=111.22910011$

A13 $=128.99407317$

A14=11.21281485 

A15 $=95.25735485$
D1 $=170.22571736$
D2 $=51.53158006$
D3=-70.02674614
D4 $=173.37563447$
D5 $=52.97417513$
D6=-68.89836919
$\mathrm{D} 7=47.08009145$
$\mathrm{D} 8=73.0212899$
D9 $=-57.58066996$
$\mathrm{D} 10=123.70087433$
D11 $=-123.8502886$
$\mathrm{D} 12=119.88252904$
D13 $=114.80092288$
D14=-43.90550302

\section{$\underline{\mathrm{HOCH}_{2} \mathrm{CH}_{2} \mathrm{CH}_{2} \mathrm{CHO} \cdot \cdot \mathrm{HO}_{2}(\mathrm{TS} 3 \mathrm{~A})}$}

Final structure in terms of initial Z-matrix:

C

$\mathrm{H}, 1, \mathrm{~B} 1$

$\mathrm{C}, 1, \mathrm{~B} 2,2, \mathrm{~A} 1$

H,3,B3,1,A2,2,D1,0

H,3,B4,1,A3,2,D2,0

C,3,B5,1,A4,2,D3,0

H,6,B6,3,A5,1,D4,0

H,6,B7,3,A6,1,D5,0

C,6,B8,3,A7,1,D6,0

O,1,B9,3,A8,6,D7,0

H,10,B10,1,A9,3,D8,0

O,9,B11,6,A10,3,D9,0

H,9,B12,6,A11,3,D10,0

H,1,B13,10,A12,9,D11,0

O,12,B14,9,A13,6,D12,0

H,15,B15,12,A14,9,D13,0

O,15,B16,12,A15,9,D14,0

Variables:

B1=1.08844415

$\mathrm{B} 2=1.52825086$

B3 $=1.08760105$

B4=1.08705211

B5 $=1.52894098$

$\mathrm{B} 6=1.08737853$

B7 $=1.08968844$

$\mathrm{B} 8=1.51140352$

B9=1.43721573 


$$
\begin{aligned}
& \text { B10 }=1.04395166 \\
& \text { B11 }=1.27780992 \\
& \text { B12 }=1.09397885 \\
& \text { B13 }=1.0878545 \\
& \text { B14 }=2.3933296 \\
& \text { B15 }=1.16099434 \\
& \text { B16 }=1.2969557 \\
& \text { A1 }=111.53848413 \\
& \text { A2 }=110.62220415 \\
& \text { A3 }=111.35014359 \\
& \text { A4 }=104.04573407 \\
& \text { A5 }=112.42552889 \\
& \text { A6 }=111.21887142 \\
& \text { A7 }=104.16460077 \\
& \text { A8 }=106.36441727 \\
& \text { A9 }=113.50928517 \\
& \text { A10 }=116.4598867 \\
& \text { A11 }=117.38683729 \\
& \text { A12 }=109.37014916 \\
& \text { A13 }=112.65833128 \\
& \text { A14 }=6.25142524 \\
& \text { A15 }=99.49854338 \\
& \text { D1 }=153.81405781 \\
& \text { D2 }=32.64013308 \\
& \text { D3 }=-89.00667553 \\
& \text { D4 }=-166.07182757 \\
& \text { D5 }=70.76209546 \\
& \text { D6 }=-48.17257461 \\
& \text { D7 }=27.25211756 \\
& \text { D8 }=112.95337762 \\
& \text { D9 }=-68.60224872 \\
& \text { D10 }=142.49230947 \\
& \text { D11 }=-121.86234033 \\
& \text { D12 }=148.28366373 \\
& \text { D13 }=115.3476834 \\
& \text { D14 }=-42.64609956 \\
&
\end{aligned}
$$

\section{$\underline{\mathrm{HOCH}_{2} \mathrm{CH}_{2} \mathrm{CH}_{2} \mathrm{CHO} \cdot \mathrm{HO}_{2}(\mathrm{PC} 3 \mathrm{~A})}$}

Final structure in terms of initial Z-matrix:
C
$\mathrm{H}, 1, \mathrm{~B} 1$
$\mathrm{C}, 1, \mathrm{~B} 2,2, \mathrm{~A} 1$
H,3,B3,1,A2,2,D1,0
$\mathrm{H}, 3, \mathrm{~B} 4,1, \mathrm{~A} 3,2, \mathrm{D} 2,0$
C,3,B5,1,A4,2,D3,0 


$$
\begin{aligned}
& \text { H,6,B6,3,A5,1,D4,0 } \\
& \text { H,6,B7,3,A6,1,D5,0 } \\
& \text { C,6,B8,3,A7,1,D6,0 } \\
& \text { O,9,B9,6,A8,3,D7,0 } \\
& \text { H,10,B10,9,A9,6,D8,0 } \\
& \text { O,9,B11,6,A10,3,D9,0 } \\
& \text { H,9,B12,6,A11,3,D10,0 } \\
& \text { H,1,B13,10,A12,9,D11,0 } \\
& \text { O,12,B14,9,A13,6,D12,0 } \\
& \text { H,12,B15,9,A14,6,D13,0 } \\
& \text { O,15,B16,12,A15,9,D14,0 } \\
& \text { Variables: } \\
& \text { B1 }=1.08897604 \\
& \text { B2 }=1.53127041 \\
& \text { B3 }=1.08682687 \\
& \text { B4 }=1.08640949 \\
& \text { B5 }=1.53106981 \\
& \text { B6=1.08553116 } \\
& \text { B7 }=1.08894562 \\
& \text { B8 }=1.51509992 \\
& \text { B9 }=1.43037791 \\
& \text { B10 }=1.62199922 \\
& \text { B11 }=1.38514512 \\
& \text { B12=1.09103749 } \\
& \text { B13=1.08850972 } \\
& \text { B14 }=2.86955119 \\
& \text { B15 }=0.96889516 \\
& \text { B16 }=1.30072055 \\
& \text { A1 }=112.37724403 \\
& \text { A2 }=110.44779975 \\
& \text { A3 }=111.94070872 \\
& \text { A4 }=102.67998606 \\
& \text { A5 }=113.59939962 \\
& \text { A6 }=111.19502841 \\
& \text { A7 }=101.22913621 \\
& \text { A8 }=103.77552298 \\
& \text { A9 }=112.64685592 \\
& \text { A10 }=108.93182581 \\
& \text { A11 }=115.23559629 \\
& \text { A12 }=109.27393004 \\
& \text { A13=92.68487475 } \\
& \text { A14 }=107.85409514 \\
& \text { A15 }=101.80660114 \\
& \text { D1 }=145.83943497 \\
& \text { D2 }=24.3333028 \\
& \text { D3 }=-97.25049497 \\
&
\end{aligned}
$$



D4 $=-155.43480283$
D5 $=80.34696328$
D6 $=-35.83574624$
$\mathrm{D} 7=40.06081128$
D8 $=-169.64462785$
D9=-78.3177451
D10 $=155.0729903$
D11 $=-115.82984399$
$\mathrm{D} 12=165.83944482$
$\mathrm{D} 13=175.06901647$
D14=-42.14859868

\section{HOCH2CH2CH2CHO••FA (RC5)}

Final structure in terms of initial Z-matrix:

C

$\mathrm{H}, 1, \mathrm{~B} 1$

$\mathrm{C}, 1, \mathrm{~B} 2,2, \mathrm{~A} 1$

H,3,B3,1,A2,2,D1,0

H,3,B4,1,A3,2,D2,0

C,3,B5,1,A4,2,D3,0

H,6,B6,3,A5,1,D4,0

H,6,B7,3,A6,1,D5,0

C,6,B8,3,A7,1,D6,0

O,1,B9,3,A8,6,D7,0

H,10,B10,1,A9,3,D8,0

O,9,B11,6,A10,3,D9,0

H,9,B12,6,A11,3,D10,0

H,1,B13,10,A12,3,D11,0

H,12,B14,9,A13,6,D12,0

C,12,B15,9,A14,6,D13,0

H,16,B16,12,A15,9,D14,0

O,16,B17,12,A16,9,D15,0

O,16,B18,12,A17,9,D16,0

Variables:

B1=1.09113414

B2 $=1.52365062$

B3 $=1.09082935$

B4=1.09010956

$\mathrm{B} 5=1.53348883$

B6 $=1.09134039$

$\mathrm{B} 7=1.0895611$

$B 8=1.50322027$

B9=1.41101524

$\mathrm{B} 10=0.96965013$

$\mathrm{B} 11=1.20979976$

B12=1.09839886 


$$
\begin{aligned}
& \text { B13 }=1.09276875 \\
& \text { B14 }=1.68060323 \\
& \text { B15 }=3.29149613 \\
& \text { B16 }=1.09511043 \\
& \text { B17 }=1.20437068 \\
& \text { B18 }=1.31087138 \\
& \text { A1 }=109.63646185 \\
& \text { A2 }=110.30870902 \\
& \text { A3 }=109.95109762 \\
& \text { A4 }=109.84005837 \\
& \text { A5 }=110.13204564 \\
& \text { A6 }=110.91261393 \\
& \text { A7 }=109.69899812 \\
& \text { A8 }=111.40972601 \\
& \text { A9 }=109.67706968 \\
& \text { A10 }=121.40327673 \\
& \text { A11 }=118.27852024 \\
& \text { A12 }=111.17422926 \\
& \text { A13 }=130.56485055 \\
& \text { A14 }=120.4335892 \\
& \text { A15 }=160.99482073 \\
& \text { A16 }=75.79531695 \\
& \text { A17 }=50.73047028 \\
& \text { D1 }=170.38344557 \\
& \text { D2 }=51.55872375 \\
& \text { D3 }=-70.00613224 \\
& \text { D4 }=173.14877524 \\
& \text { D5 }=52.69835692 \\
& \text { D6 }=-69.12078825 \\
& \text { D7 }=47.31174698 \\
& \text { D8 }=75.50290564 \\
& \text { D9 }=-60.03018401 \\
& \text { D10 }=121.01549802 \\
& \text { D11 }=-123.63896567 \\
& \text { D12 }=143.21950324 \\
& \text { D13 }=115.29379181 \\
& \text { D14 }=147.64961474 \\
& \text { D15 }=-46.79655243 \\
& \text { D16 }=127.34833996
\end{aligned}
$$

\section{HOCH2CH2CH2CHO•FA (TS5)}

Final structure in terms of initial Z-matrix:

C

$\mathrm{H}, 1, \mathrm{~B} 1$

$\mathrm{C}, 1, \mathrm{~B} 2,2, \mathrm{~A} 1$

H,3,B3,1,A2,2,D1,0 


$$
\begin{aligned}
& \text { H,3,B4,1,A3,2,D2,0 } \\
& \text { C,3,B5,1,A4,2,D3,0 } \\
& \text { H,6,B6,3,A5,1,D4,0 } \\
& \text { H,6,B7,3,A6,1,D5,0 } \\
& \text { C,6,B8,3,A7,1,D6,0 } \\
& \text { O,1,B9,3,A8,6,D7,0 } \\
& \text { H,10,B10,1,A9,3,D8,0 } \\
& \text { O,9,B11,6,A10,3,D9,0 } \\
& \text { H,9,B12,6,A11,3,D10,0 } \\
& \text { H,1,B13,10,A12,9,D11,0 } \\
& \text { H,12,B14,9,A13,6,D12,0 } \\
& \text { C,12,B15,9,A14,6,D13,0 } \\
& \text { H,16,B16,12,A15,9,D14,0 } \\
& \text { O,16,B17,12,A16,9,D15,0 } \\
& \text { O,16,B18,12,A17,9,D16,0 } \\
& \text { Variables: } \\
& \text { B1 }=1.0889145 \\
& \text { B2 }=1.53009067 \\
& \text { B3 }=1.08770798 \\
& \text { B4 }=1.087154 \\
& \mathrm{~B} 5=1.52902435 \\
& \text { B6 }=1.08766495 \\
& \text { B7 }=1.08945061 \\
& \text { B8 }=1.50910641 \\
& \mathrm{~B} 9=1.43212554 \\
& \text { B10 }=1.03577915 \\
& \mathrm{~B} 11=1.27042845 \\
& \text { B12 }=1.0934667 \\
& \mathrm{~B} 13=1.08810614 \\
& \mathrm{~B} 14=1.22762315 \\
& \text { B15 }=3.08622029 \\
& \text { B16 }=1.09688909 \\
& \mathrm{~B} 17=1.23593922 \\
& \mathrm{~B} 18=1.26822602 \\
& \text { A1 }=111.32686983 \\
& \text { A2 }=110.66554869 \\
& \text { A3 }=111.41395751 \\
& \text { A4 }=104.3319156 \\
& \text { A5 }=112.31712779 \\
& \text { A6 }=111.22447115 \\
& \text { A7 }=104.43604754 \\
& \text { A8 }=106.54788224 \\
& \text { A9 }=113.12838976 \\
& \text { A10 }=116.68081003 \\
& \text { A11 }=118.0091464 \\
& \text { A12=109.52283441 }
\end{aligned}
$$



A13 $=114.26062645$
A14 $=104.20221016$
$\mathrm{A} 15=159.25008309$
$\mathrm{A} 16=81.15027484$
A17 $=46.44914492$
D1 $=152.30537985$
D2 $=31.12751212$
D3 $=-90.47585667$
D4 $=-167.00247239$
D5 $=69.87864653$
D6 $=-49.03543675$
D7 $=26.0869336$
$\mathrm{D} 8=116.14196211$
D9 $=-67.57217287$
$\mathrm{D} 10=141.62283438$
D11=-119.87007397
D12 $=170.11661776$
$\mathrm{D} 13=145.89215514$
D14 $=149.66640494$
D15 $=-50.19541652$
D16 $=120.91339665$

\section{HOCH2CH2CH2CHO••FA (PC5)}

Final structure in terms of initial Z-matrix:

C

$\mathrm{H}, 1, \mathrm{~B} 1$

$\mathrm{C}, 1, \mathrm{~B} 2,2, \mathrm{~A} 1$

H,3,B3,1,A2,2,D1,0

$\mathrm{H}, 3, \mathrm{~B} 4,1, \mathrm{~A} 3,2, \mathrm{D} 2,0$

$\mathrm{C}, 3, \mathrm{~B} 5,1, \mathrm{~A} 4,2, \mathrm{D} 3,0$

$\mathrm{H}, 6, \mathrm{~B} 6,3, \mathrm{~A} 5,1, \mathrm{D} 4,0$

H,6,B7,3,A6,1,D5,0

C,6,B8,3,A7,1,D6,0

O,9,B9,6,A8,3,D7,0

H,10,B10,9,A9,6,D8,0

O,9,B11,6,A10,3,D9,0

H,9,B12,6,A11,3,D10,0

H,1,B13,10,A12,9,D11,0

H,12,B14,9,A13,6,D12,0

C,10,B15,9,A14,6,D13,0

H,16,B16,10,A15,9,D14,0

O,16,B17,10,A16,9,D15,0

O,16,B18,10,A17,9,D16,0

Variables:

B1 $=1.08941762$

B2 $=1.53134203$ 


$$
\begin{aligned}
& \text { B3 }=1.08707901 \\
& \text { B4 }=1.08654055 \\
& \text { B5 }=1.53060557 \\
& \text { B6 }=1.08551262 \\
& \text { B7 }=1.0891296 \\
& \text { B8 }=1.51633285 \\
& \text { B9 }=1.43148567 \\
& \text { B10 }=1.6391461 \\
& \text { B11 }=1.38266717 \\
& \text { B12 }=1.09091314 \\
& \text { B13 }=1.08868602 \\
& \text { B14 }=0.96958651 \\
& \text { B15 }=3.3418406 \\
& \text { B16 }=1.09526928 \\
& \text { B17 }=1.30932467 \\
& \text { B18 }=1.20574112 \\
& \text { A1 }=112.2362971 \\
& \text { A2 }=110.44400641 \\
& \text { A3 }=111.98147312 \\
& \text { A4 }=102.52226065 \\
& \text { A5 }=113.65916216 \\
& \text { A6 }=111.09693752 \\
& \text { A7 }=101.18853109 \\
& \text { A8 }=103.5739728 \\
& \text { A9 }=116.46556135 \\
& \text { A10 }=109.06474244 \\
& \text { A11 }=115.17502851 \\
& \text { A12 }=109.3341874 \\
& \text { A13 }=107.65284559 \\
& \text { A14 }=95.37665207 \\
& \text { A15 }=158.81691201 \\
& \text { A16 }=47.36452696 \\
& \text { A17 }=78.66354325 \\
& \text { D1 }=146.4674333 \\
& \text { D2 }=24.92511446 \\
& \text { D3 }=-96.73171475 \\
& \text { D4 }=-155.91844916 \\
& \text { D5 }=79.8476072 \\
& \text { D6 }=-36.36782107 \\
& \text { D7 }=40.27481687 \\
& \text { D8 }=-173.34051268 \\
& \text { D9 }=-78.3417247 \\
& \text { D10 }=155.11385168 \\
& \text { D11 }=-116.40409108 \\
& \text { D12 }=-178.02293683 \\
& \text { D13 }=-171.42118493 \\
&
\end{aligned}
$$




$$
\begin{aligned}
& \mathrm{D} 14=-168.33510695 \\
& \mathrm{D} 15=-173.69637836 \\
& \mathrm{D} 16=7.89856037
\end{aligned}
$$

\section{$\underline{\mathrm{HOCH}} \underline{\mathrm{CH}}_{2} \underline{\mathrm{CH}}_{2} \underline{\mathrm{CHO} \cdot \mathrm{FA}}$ (RC5A)}

Final structure in terms of initial Z-matrix:

C

$\mathrm{H}, 1, \mathrm{~B} 1$

$\mathrm{C}, 1, \mathrm{~B} 2,2, \mathrm{~A} 1$

$\mathrm{H}, 3, \mathrm{~B} 3,1, \mathrm{~A} 2,2, \mathrm{D} 1,0$

H,3,B4,1,A3,2,D2,0

C,3,B5,1,A4,2,D3,0

H,6,B6,3,A5,1,D4,0

H,6,B7,3,A6,1,D5,0

C,6,B8,3,A7,1,D6,0

O,1,B9,3,A8,6,D7,0

H,10,B10,1,A9,3,D8,0

O,9,B11,6,A10,3,D9,0

C,12,B12,9,A11,6,D10,0

H,13,B13,12,A12,9,D11,0

O,13,B14,12,A13,9,D12,0

O,13,B15,12,A14,9,D13,0

H,16,B16,13,A15,12,D14,0

H,9,B17,6,A16,3,D15,0

H,1,B18,10,A17,3,D16,0

Variables:

B1 $=1.09108457$

B2 $=1.52461288$

B3 $=1.09064797$

B4 $=1.09026572$

B5 $=1.52891127$

$\mathrm{B} 6=1.09271816$

$\mathrm{B} 7=1.08985287$

$\mathrm{B} 8=1.50455899$

B9 $=1.41107694$

$\mathrm{B} 10=0.96755514$

$\mathrm{B} 11=1.20844823$

B12 $=3.34260503$

$\mathrm{B} 13=1.09519497$

B14 $=1.20389166$

B15 $=1.31181094$

$\mathrm{B} 16=0.9902011$

B17 $=1.09943576$

B18=1.09252324

A1=109.66449715

A2 $=110.23785992$ 


$$
\begin{aligned}
& \text { A3 }=109.85708021 \\
& \text { A4 }=109.88113652 \\
& \text { A5 }=110.4284526 \\
& \text { A6 }=110.64572329 \\
& \text { A7 }=111.95323144 \\
& \text { A8 }=111.38357775 \\
& \text { A9=106.91538717 } \\
& \mathrm{A} 10=123.58929381 \\
& \mathrm{~A} 11=124.73767101 \\
& \text { A12 }=159.63622352 \\
& \text { A13 }=77.35719899 \\
& \text { A14 }=48.87179106 \\
& \mathrm{~A} 15=110.65095872 \\
& \text { A16 }=117.30849694 \\
& \text { A17=110.92920276 } \\
& \text { D1 }=169.52228563 \\
& \text { D2 }=51.45302662 \\
& \text { D3 }=-69.80558371 \\
& \text { D4 }=172.92028016 \\
& \text { D5 }=53.39774542 \\
& \text { D6=-68.71142126 } \\
& \text { D7 }=47.73944759 \\
& \text { D8 }=74.75752772 \\
& \text { D9 }=-54.11255588 \\
& \text { D10 }=55.96710496 \\
& \text { D11=-150.052264 } \\
& \text { D12 }=40.07288524 \\
& \text { D13 }=-134.95061585 \\
& \text { D14=3.63751241 } \\
& \text { D15 }=129.19652926 \\
& \text { D16=-123.16119878 }
\end{aligned}
$$

\section{$\underline{\mathrm{HOCH}_{2}} \underline{\mathrm{CH}}_{2} \underline{\mathrm{CH}}_{2} \underline{\mathrm{CHO} \cdot \bullet \mathrm{FA}}$ (TS5A)}

Final structure in terms of initial Z-matrix:

C

$\mathrm{H}, 1, \mathrm{~B} 1$

$\mathrm{C}, 1, \mathrm{~B} 2,2, \mathrm{~A} 1$

H,3,B3,1,A2,2,D1,0

H,3,B4,1,A3,2,D2,0

C,3,B5,1,A4,2,D3,0

H,6,B6,3,A5,1,D4,0

H,6,B7,3,A6,1,D5,0

C,6,B8,3,A7,1,D6,0

O,1,B9,3,A8,6,D7,0

H,10,B10,1,A9,3,D8,0

O,9,B11,6,A10,3,D9,0 
C,12,B12,9,A11,6,D10,0

H,13,B13,12,A12,9,D11,0

O,13,B14,12,A13,9,D12,0

O,13,B15,12,A14,9,D13,0

H,12,B16,9,A15,6,D14,0

H,9,B17,6,A16,3,D15,0

H,1,B18,10,A17,9,D16,0

Variables:

B1 $=1.08919924$

B2 $=1.51948047$

B3=1.08949982

B4 $=1.08771231$

B5 $=1.52792997$

B6 $=1.08771816$

B7 $=1.09002258$

B8 $=1.52419642$

B9 $=1.42984779$

$\mathrm{B} 10=1.03403379$

$\mathrm{B} 11=1.27116862$

B12 $=3.05548877$

B13 $=1.09771055$

$\mathrm{B} 14=1.23747907$

B15 $=1.2670064$

$\mathrm{B} 16=1.18737479$

$\mathrm{B} 17=1.08996952$

B18 $=1.0882805$

A1 $=111.82774284$

A2 $=109.84916005$

A3 $=112.28148759$

A4 $=103.73952003$

A5 $=112.52128038$

A6 $=109.93806746$

A7 $=108.68275108$

A8 $=105.5704424$

A9 $=105.22100578$

A10 $=121.02895994$

A11 $=108.95591451$

A12 $=159.43918358$

A13 $=80.04536613$

A14 $=47.71737887$

A15 $=117.00523143$

A16 $=115.70509004$

A17 $=108.6648077$

$\mathrm{D} 1=167.38478657$

$\mathrm{D} 2=46.50662697$

D3 $=-74.95645771$ 


$$
\begin{aligned}
& \text { D4 }=-162.27778662 \\
& \text { D5 }=76.43763417 \\
& \text { D6 }=-42.74767376 \\
& \text { D7 }=41.64750379 \\
& \text { D8 }=81.9197349 \\
& \text { D9 }=-89.07521398 \\
& \text { D10 }=72.02652146 \\
& \text { D11 }=-156.80026752 \\
& \text { D12 }=49.67341124 \\
& \text { D13 }=-118.523169 \\
& \text { D14 }=44.73397689 \\
& \text { D15 }=124.38235137 \\
& \text { D16 }=-147.31305187
\end{aligned}
$$

\section{$\underline{\mathrm{HOCH}} 2 \mathrm{CH}_{2} \mathrm{CH}_{2} \mathrm{CHO} \cdot \cdot \mathrm{FA}$ (PC5A)}

Final structure in terms of initial Z-matrix:

C

$\mathrm{H}, 1, \mathrm{~B} 1$

$\mathrm{C}, 1, \mathrm{~B} 2,2, \mathrm{~A} 1$

H,3,B3,1,A2,2,D1,0

$\mathrm{H}, 3, \mathrm{~B} 4,1, \mathrm{~A} 3,2, \mathrm{D} 2,0$

C,3,B5,1,A4,2,D3,0

H,6,B6,3,A5,1,D4,0

H,6,B7,3,A6,1,D5,0

C,6,B8,3,A7,1,D6,0

O,1,B9,3,A8,6,D7,0

H,10,B10,1,A9,3,D8,0

O,9,B11,6,A10,3,D9,0

C,12,B12,9,A11,6,D10,0

H,13,B13,12,A12,9,D11,0

O,13,B14,12,A13,9,D12,0

O,13,B15,12,A14,9,D13,0

H,12,B16,9,A15,6,D14,0

H,9,B17,6,A16,3,D15,0

H,1,B18,10,A17,9,D16,0

Variables:

B1 $=1.09099141$

$\mathrm{B} 2=1.52238029$

B3 $=1.08895722$

B4 $=1.08658739$

B5 $=1.52775765$

B6 $=1.08582277$

B7 $=1.09041452$

B8 $=1.52566033$

B9 $=1.43674734$

B10 $=1.68674585$ 


$$
\begin{aligned}
& \text { B11 }=1.38601255 \\
& \text { B12 }=3.3139158 \\
& \text { B13 }=1.09511959 \\
& \text { B14 }=1.31017977 \\
& \text { B15 }=1.2066851 \\
& \text { B16 }=0.96873633 \\
& \text { B17 }=1.0874428 \\
& \text { B18 }=1.08919457 \\
& \text { A1 }=111.83189087 \\
& \text { A2 }=110.01703051 \\
& \text { A3 }=112.39061004 \\
& \text { A4 }=101.1942036 \\
& \text { A5 }=114.55121887 \\
& \text { A6 }=110.34803133 \\
& \text { A7 }=102.24566984 \\
& \text { A8 }=105.73018263 \\
& \text { A9 }=106.8189066 \\
& \text { A10 }=114.25028402 \\
& \text { A11 }=103.28510967 \\
& \text { A12 }=150.5597582 \\
& \text { A13 }=75.59702801 \\
& \text { A14 }=57.81493527 \\
& \text { A15 }=109.13793923 \\
& \text { A16 }=113.68223437 \\
& \text { A17 }=108.41200085 \\
& \text { D1 }=155.76775931 \\
& \text { D2 }=34.39244437 \\
& \text { D3 }=-86.87580334 \\
& \text { D4 }=-158.69932078 \\
& \text { D5 }=77.7412989 \\
& \text { D6 }=-38.66248687 \\
& \text { D7 }=30.6730489 \\
& \text { D8 }=112.65865567 \\
& \text { D9 }=-87.19696959 \\
& \text { D10 }=103.82037852 \\
& \text { D11 }=163.21835533 \\
& \text { D12 }=54.84383284 \\
& \text { D13 }=-96.16383823 \\
& \text { D14 }=62.47083298 \\
& \text { D15 }=150.50037934 \\
& \text { D16 }=-131.99097042 \\
&
\end{aligned}
$$

\section{2-OH-THF••FA (RC6)}

Final structure in terms of initial Z-matrix:

C

H,1,B1 
$\mathrm{C}, 1, \mathrm{~B} 2,2, \mathrm{~A} 1$

H,3,B3,1,A2,2,D1,0

H,3,B4,1,A3,2,D2,0

C,3,B5,1,A4,2,D3,0

H,6,B6,3,A5,1,D4,0

H,6,B7,3,A6,1,D5,0

C,6,B8,3,A7,1,D6,0

O,9,B9,6,A8,3,D7,0

O,9,B10,6,A9,3,D8,0

H,9,B11,6,A10,3,D9,0

H,1,B12,10,A11,9,D10,0

H,11,B13,9,A12,6,D11,0

C,11,B14,9,A13,6,D12,0

H,15,B15,11,A14,9,D13,0

O,15,B16,11,A15,9,D14,0

O,15,B17,11,A16,9,D15,0

H,18,B18,15,A17,11,D16,0

Variables:

B1 $=1.08623284$

B2 $=1.53852529$

B3 $=1.0866842$

B4 $=1.0865471$

B5 $=1.54152107$

B6 $=1.08632459$

$\mathrm{B} 7=1.08710721$

B8 $=1.52020955$

B9 $=1.39023358$

$\mathrm{B} 10=1.42534324$

B11 $=1.08878854$

$\mathrm{B} 12=1.09165642$

B13 $=0.96210844$

B14 $=3.51243923$

B15 $=1.09628603$

$\mathrm{B} 16=1.19735667$

$\mathrm{B} 17=1.32273466$

B18 $=0.98633863$

A1 $=113.87063123$

A2 $=111.34347663$

A3 $=111.28512641$

A4 $=104.00869224$

A5 $=112.8641127$

A6 $=111.12156147$

A7 $=103.16007696$

A8 $=106.37497298$

A9 $=108.00792606$

A10 $=115.13642445$ 

$\mathrm{A} 11=109.52210421$
A12 $=108.00952411$
A13 $=113.75190934$
A14 $=154.4589431$
A15 $=81.7347763$
A16 $=43.96174255$
A17 $=110.38700848$
D1 $=108.91347877$
D2 $=-11.52067348$
D3 $=-131.11051617$
D4 $=-128.50849417$
D5 $=108.45191231$
D6 $=-8.06102915$
D7 $=27.93810083$
D8 $=-90.71508111$
D9 $=147.05051029$
D10=-87.56606094
D11 $=171.46768076$
$\mathrm{D} 12=22.25788558$
D13 $=127.38022154$
D14 $=-57.32096833$
D15 $=119.21418362$
D16=-1.21682812

\section{2-OH-THF••FA (TS6)}

Final structure in terms of initial Z-matrix:

C

$\mathrm{H}, 1, \mathrm{~B} 1$

C,1,B2,2,A1

H,3,B3, 1,A2,2,D1,0

H,3,B4,1,A3,2,D2,0

C,3,B5,1,A4,2,D3,0

H,6,B6,3,A5,1,D4,0

H,6,B7,3,A6,1,D5,0

$\mathrm{C}, 6, \mathrm{~B} 8,3, \mathrm{~A} 7,1, \mathrm{D} 6,0$

O,9,B9,6,A8,3,D7,0

O,9,B10,6,A9,3,D8,0

H,9,B11,6,A10,3,D9,0

H,1,B12,10,A11,9,D10,0

H,11,B13,9,A12,6,D11,0

C,6,B14,3,A13,1,D12,0

H,15,B15,6,A14,3,D13,0

O,15,B16,6,A15,3,D14,0

O,15,B17,6,A16,3,D15,0

H,11,B18,9,A17,6,D16,0

Variables: 

B1 $=1.08424991$
B2 $=1.52757235$
B3 $=1.08647773$
B4 $=1.08800828$
B5 $=1.53650751$
B6 $=1.17521086$
B7 $=1.08634198$
B8 $=1.42666627$
B9=1.26741473
$\mathrm{B} 10=2.50398768$
$\mathrm{B} 11=1.07915451$
$\mathrm{B} 12=1.08510773$
$\mathrm{B} 13=0.95813134$
B14=3.24569537
B15=1.10553953
B16=1.24786699
$\mathrm{B} 17=1.2522842$
B18 $=0.99047332$
A1=115.6963201
A2 $=110.79551494$
A3 $=111.65089211$
A4 $=102.44922163$
A5 $=106.89200634$
A6 $=118.65465721$
A7 $=103.53537433$
A8 $=115.57834284$
A9 $=103.12310694$
A10 $=127.96394182$
A11 $=105.2313533$
A12 $=130.74119551$
$\mathrm{A} 13=81.91464063$
A14=153.04802971
$\mathrm{A} 15=54.02532953$
A16 $=77.12535347$
A17 $=86.35284502$
D1 $=106.49116578$
D2=-15.66213915
D3 $=-135.43124194$
D4=-96.14460676
D5 $=145.76025691$
D6=18.23696462
D7 $=-10.51952643$
D8 $=-116.96612001$
D9 $=167.44719141$
D10 $=-103.79374938$
D11 $=160.90437351$ 
$\mathrm{D} 12=-84.44822122$

D13 $=-47.42281373$

D14=-125.62596926

D15 $=78.15903985$

D16 $=53.48857543$

\section{$\underline{\text { 2-OH-THF••FA (PC6) }}$}

Final structure in terms of initial Z-matrix:

C

$\mathrm{H}, 1, \mathrm{~B} 1$

$\mathrm{C}, 1, \mathrm{~B} 2,2, \mathrm{~A} 1$

H,3,B3,1,A2,2,D1,0

H,3,B4,1,A3,2,D2,0

C,3,B5,1,A4,2,D3,0

H,6,B6,3,A5,1,D4,0

H,6,B7,3,A6,1,D5,0

C,6,B8,3,A7,1,D6,0

O,9,B9,6,A8,3,D7,0

O,9,B10,6,A9,3,D8,0

H,9,B11,6,A10,3,D9,0

H,1,B12,10,A11,9,D10,0

H,11,B13,9,A12,6,D11,0

C,11,B14,9,A13,6,D12,0

H,15,B15,11,A14,9,D13,0

O,15,B16,11,A15,9,D14,0

O,15,B17,11,A16,9,D15,0

H,11,B18,9,A17,6,D16,0

Variables:

B1=1.08926543

B2 $=1.53896309$

B3 $=1.09001829$

B4=1.08863645

$\mathrm{B} 5=1.50970006$

$\mathrm{B} 6=4.08875184$

$\mathrm{B} 7=1.07629756$

$\mathrm{B} 8=1.32254123$

$\mathrm{B} 9=1.3666476$

$\mathrm{B} 10=3.70519005$

$\mathrm{B} 11=1.07819$

B12 $=1.08592697$

$\mathrm{B} 13=0.96354579$

B14=3.15220385

B15=1.09543554

B16=1.31696014

B17 $=1.20401558$

B18 $=0.97052989$ 


$$
\begin{aligned}
& \text { A1 }=112.05421332 \\
& \text { A2 }=111.54322249 \\
& \text { A3 }=111.56436606 \\
& \text { A4 }=101.27603531 \\
& \text { A5 }=94.27232509 \\
& \text { A6 }=125.66493028 \\
& \text { A7 }=109.01275348 \\
& \text { A8 }=114.7767766 \\
& \text { A9 }=129.7432437 \\
& \text { A10 }=130.31485539 \\
& \text { A11 }=107.70839805 \\
& \text { A12 }=101.93918494 \\
& \text { A13 }=53.90459756 \\
& \text { A14 }=166.06493016 \\
& \text { A15 }=57.0341399 \\
& \text { A16 }=69.04988848 \\
& \text { A17 }=30.29522267 \\
& \text { D1 }=135.4544696 \\
& \text { D2 }=14.15863508 \\
& \text { D3 }=-105.97171649 \\
& \text { D4 }=-50.39822372 \\
& \text { D5 }=174.27326092 \\
& \text { D6 }=-7.62399513 \\
& \text { D7 }=1.34505226 \\
& \text { D8 }=-44.90249608 \\
& \text { D9 }=-177.3782117 \\
& \text { D10 }=-132.19588174 \\
& \text { D11 }=10.26663601 \\
& \text { D12 }=-42.96851701 \\
& \text { D13=-54.95731859 } \\
& \text { D14 }=-90.09499762 \\
& \text { D15 }=97.66837573 \\
& \text { D16 }=106.10191227 \\
&
\end{aligned}
$$

\section{$\mathrm{HOCH}_{2} \mathrm{CH}_{2} \mathrm{CH}_{2} \mathrm{CHO} \cdot \cdot \mathrm{HNO}_{3}(\mathrm{RC} 7)$}

Final structure in terms of initial Z-matrix:

C

$\mathrm{H}, 1, \mathrm{~B} 1$

C,1,B2,2,A1

H,3,B3,1,A2,2,D1,0

H,3,B4,1,A3,2,D2,0

C,3,B5,1,A4,2,D3,0

H,6,B6,3,A5,1,D4,0

H,6,B7,3,A6,1,D5,0

C,6,B8,3,A7,1,D6,0

O,1,B9,3,A8,6,D7,0 


$$
\begin{aligned}
& \text { H,10,B10,1,A9,3,D8,0 } \\
& \text { O,9,B11,6,A10,3,D9,0 } \\
& \text { H,9,B12,6,A11,3,D10,0 } \\
& \text { H,1,B13,10,A12,3,D11,0 } \\
& \text { N,12,B14,9,A13,6,D12,0 } \\
& \text { O,15,B15,12,A14,9,D13,0 } \\
& \text { O,15,B16,12,A15,9,D14,0 } \\
& \text { O,15,B17,12,A16,9,D1,0 } \\
& \text { H,18,B18,15,A17,12,D16,0 } \\
& \text { Variables: } \\
& \text { B1 }=1.09046537 \\
& \text { B2 }=1.52281071 \\
& \text { B3 }=1.09085288 \\
& \text { B4 }=1.08983469 \\
& \text { B5 }=1.53461555 \\
& \text { B6 }=1.09114885 \\
& \text { B7 }=1.08926335 \\
& \text { B8 }=1.50079610 \\
& \text { B9 }=1.41402804 \\
& \text { B10 }=0.96522822 \\
& \text { B11 }=1.21239971 \\
& \text { B12 }=1.09765098 \\
& \text { B13 }=1.09215900 \\
& \text { B14=3.23702372 } \\
& \text { B15 }=1.18843878 \\
& \text { B16=1.21396524 } \\
& \text { B17 }=1.34127669 \\
& \text { B18=1.00753204 } \\
& \text { A1 }=109.77658867 \\
& \text { A2 }=110.40394955 \\
& \text { A3 }=109.82697500 \\
& \text { A4 }=110.03344892 \\
& \text { A5 }=110.01383912 \\
& \text { A6 }=110.96851232 \\
& \text { A7 }=109.51564432 \\
& \text { A8 }=111.47689923 \\
& \text { A9 }=110.22515555 \\
& \text { A10 }=121.21121282 \\
& \text { A11 }=118.58712181 \\
& \text { A12 }=111.16288652 \\
& \text { A13 }=117.50454546 \\
& \text { A14 }=165.53385381 \\
& \text { A15 }=67.28204838 \\
& \text { A16=49.94544325 } \\
& \text { A17=106.96892956 } \\
& \text { D1 }=171.01799713 \\
&
\end{aligned}
$$




$$
\begin{aligned}
& \mathrm{D} 2=52.29981364 \\
& \mathrm{D} 3=-69.14172158 \\
& \mathrm{D} 4=174.00079627 \\
& \mathrm{D} 5=53.49844663 \\
& \mathrm{D} 6=-68.29027508 \\
& \mathrm{D} 7=47.82039895 \\
& \mathrm{D} 8=74.33981564 \\
& \mathrm{D} 9=-60.58034768 \\
& \mathrm{D} 10=119.93679087 \\
& \mathrm{D} 11=-123.87850265 \\
& \mathrm{D} 12=127.65354653 \\
& \mathrm{D} 13=113.61238208 \\
& \mathrm{D} 14=-71.18901035 \\
& \mathrm{D} 15=107.33236425 \\
& \mathrm{D} 16=0.21372290
\end{aligned}
$$

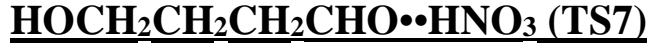

Final structure in terms of initial Z-matrix:

C

$\mathrm{H}, 1, \mathrm{~B} 1$

$\mathrm{C}, 1, \mathrm{~B} 2,2, \mathrm{~A} 1$

H,3,B3,1,A2,2,D1,0

H,3,B4,1,A3,2,D2,0

C,3,B5,1,A4,2,D3,0

H,6,B6,3,A5,1,D4,0

H,6,B7,3,A6,1,D5,0

C,6,B8,3,A7,1,D6,0

O,1,B9,3,A8,6,D7,0

H,10,B10,1,A9,3,D8,0

O,9,B11,6,A10,3,D9,0

H,9,B12,6,A11,3,D10,0

H,1,B13,10,A12,9,D11,0

N,12,B14,9,A13,6,D12,0

O,15,B15,12,A14,9,D13,0

O,15,B16,12,A15,9,D14,0

O,15,B17,12,A16,9,D15,0

H,12,B18,9,A17,6,D16,0

Variables:

$\mathrm{B} 1=1.08863828$

B2 $=1.52785799$

B3 $=1.08809315$

B4 $=1.08725016$

B5 $=1.53065313$

B6 $=1.08850474$

B7 $=1.08893039$ 


$$
\begin{aligned}
& \mathrm{B} 8=1.50107493 \\
& \text { B9 }=1.43147068 \\
& \text { B10 }=0.99952299 \\
& \mathrm{~B} 11=1.26263067 \\
& \text { B12 }=1.09075948 \\
& \mathrm{~B} 13=1.08779778 \\
& \text { B14 }=3.09696523 \\
& \mathrm{~B} 15=1.19400614 \\
& \text { B16 }=1.24635358 \\
& \mathrm{~B} 17=1.29085785 \\
& \text { B18=1.1616522 } \\
& \text { A1 }=111.09009158 \\
& \text { A2 }=110.69981293 \\
& \text { A3 }=111.34165258 \\
& \text { A4 }=104.93364239 \\
& \text { A5 }=111.92320923 \\
& \text { A6 }=111.29941044 \\
& \text { A7 }=105.46247065 \\
& \text { A8 }=107.12352098 \\
& \text { A9 }=112.407997 \\
& \text { A10 }=117.37473536 \\
& \text { A11 }=119.77073561 \\
& \text { A12 }=109.80270608 \\
& \text { A13 }=107.87350748 \\
& \text { A14 }=162.15786658 \\
& \text { A15 }=72.87926777 \\
& \text { A16 }=46.34757804 \\
& \text { A17 }=112.20980705 \\
& \text { D1 }=156.72433125 \\
& \mathrm{D} 2=35.71535459 \\
& \text { D3 }=-85.67642773 \\
& \text { D4 }=-170.09824815 \\
& \text { D5 }=67.1629611 \\
& \text { D6 }=-52.1540651 \\
& \text { D7 }=30.59849197 \\
& \text { D8 }=104.86397666 \\
& \text { D9 }=-65.96731937 \\
& \text { D10 }=136.94482569 \\
& \text { D11 }=-125.35185765 \\
& \text { D12 }=144.37441957 \\
& \text { D13 }=140.47132503 \\
& \text { D14 }=-66.48729864 \\
& \text { D15 }=102.00300873 \\
& \text { D16 }=169.66760368
\end{aligned}
$$

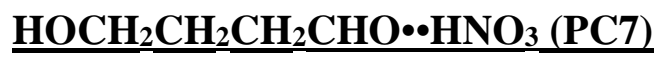


Final structure in terms of initial Z-matrix:

$$
\begin{aligned}
& \text { C } \\
& \mathrm{H}, 1, \mathrm{~B} 1 \\
& \mathrm{C}, 1, \mathrm{~B} 2,2, \mathrm{~A} 1 \\
& \text { H,3,B3,1,A2,2,D1,0 } \\
& \text { H,3,B4,1,A3,2,D2,0 } \\
& \text { C,3,B5,1,A4,2,D3,0 } \\
& \text { H,6,B6,3,A5,1,D4,0 } \\
& \text { H,6,B7,3,A6,1,D5,0 } \\
& \text { C,6,B8,3,A7,1,D6,0 } \\
& \text { O,9,B9,6,A8,3,D7,0 } \\
& \text { H,10,B10,9,A9,6,D8,0 } \\
& \text { O,9,B11,6,A10,3,D9,0 } \\
& \text { H,9,B12,6,A11,3,D10,0 } \\
& \text { H,1,B13,10,A12,9,D11,0 } \\
& \text { N,10,B14,9,A13,6,D12,0 } \\
& \text { O,15,B15,10,A14,9,D13,0 } \\
& \text { O,15,B16,10,A15,9,D14,0 } \\
& \text { O,15,B17,10,A16,9,D15,0 } \\
& \text { H,12,B18,9,A17,6,D16,0 } \\
& \text { Variables: } \\
& \text { B1=1.08886098 } \\
& \text { B2 }=1.53009412 \\
& \text { B3 }=1.08707140 \\
& \text { B4 }=1.08629551 \\
& \text { B5 }=1.53090166 \\
& \text { B6 }=1.08530867 \\
& \text { B7 }=1.08903125 \\
& B 8=1.51539642 \\
& \text { B9=1.43195665 } \\
& \mathrm{B} 10=1.55141006 \\
& \mathrm{~B} 11=1.38605364 \\
& \text { B12 }=1.08929323 \\
& \mathrm{~B} 13=1.08838149 \\
& \text { B14=3.27772019 } \\
& \mathrm{B} 15=1.18811539 \\
& \text { B16 }=1.33801838 \\
& \mathrm{~B} 17=1.21646310 \\
& \text { B18 }=0.96439211 \\
& \text { A1=112.40799592 } \\
& \text { A2 }=110.51160302 \\
& \text { A3 }=111.94011018 \\
& \text { A4=102.55691233 } \\
& \text { A5 }=113.70371286 \\
& \text { A6 }=111.10851443 \\
& \text { A } 7=101.34637710
\end{aligned}
$$




$$
\begin{aligned}
& \text { A8 }=103.46790532 \\
& \text { A9 }=118.67640383 \\
& \text { A10 }=108.84464086 \\
& \text { A11 }=115.48366062 \\
& \text { A12 }=109.07689495 \\
& \text { A13 }=98.73174081 \\
& \text { A14 }=163.17293736 \\
& \text { A15 }=47.18230564 \\
& \text { A16 }=69.78434479 \\
& \text { A17 }=107.94230005 \\
& \text { D1 }=146.63762475 \\
& \text { D2 }=25.08563270 \\
& D 3=-96.42060838 \\
& \text { D4 }=-156.18370132 \\
& \text { D5 }=79.61758489 \\
& \text { D6 }=-36.49678084 \\
& \text { D7 }=40.17973022 \\
& \text { D8 }=-168.95674994 \\
& \text { D9 }=-78.08682463 \\
& \text { D10 }=155.31313471 \\
& \text { D11 }=-116.54805161 \\
& \text { D12 }=-172.57089506 \\
& \text { D13 }=172.55949390 \\
& \text { D14 }=170.11316717 \\
& \text { D15 }=-9.29543684 \\
& \text { D16 }=-176.71741252
\end{aligned}
$$

\section{$\underline{\mathrm{HOCH}_{2} \mathrm{CH}_{2} \mathrm{CH}_{2} \mathrm{CHO} \cdot \cdot \mathrm{HNO}_{3}(\mathrm{RC} 7 \mathrm{~A})}$}

Final structure in terms of initial Z-matrix:

C

$\mathrm{H}, 1, \mathrm{~B} 1$

$\mathrm{C}, 1, \mathrm{~B} 2,2, \mathrm{~A} 1$

H,3,B3,1,A2,2,D1,0

H,3,B4,1,A3,2,D2,0

C,3,B5,1,A4,2,D3,0

H,6,B6,3,A5,1,D4,0

H,6,B7,3,A6,1,D5,0

C,6,B8,3,A7,1,D6,0

O,1,B9,3,A8,6,D7,0

H,10,B10,1,A9,3,D8,0

O,9,B11,6,A10,3,D9,0

N,12,B12,9,A11,6,D10,0

O,13,B13,12,A12,9,D11,0

O,13,B14,12,A13,9,D12,0

O,13,B15,12,A14,9,D13,0 
H,16,B16,13,A15,12,D14,0

H,9,B17,6,A16,3,D15,0

H,1,B18,10,A17,3,D16,0

Variables:

B1=1.09041563

B2 $=1.52545435$

B3 $=1.08967177$

B4 $=1.08986557$

B5 $=1.52800029$

B6 $=1.09330235$

B7 $=1.08968767$

$\mathrm{B} 8=1.50237618$

B9 $=1.41370988$

B10 $=0.96364467$

$\mathrm{B} 11=1.21046128$

B12 $=3.30194463$

$\mathrm{B} 13=1.21290043$

B14=1.18863005

$\mathrm{B} 15=1.34200331$

B16 $=1.00454119$

B17 $=1.0987596$

$\mathrm{B} 18=1.0919738$

A1 $=109.64533916$

A2 $=110.59684517$

A3 $=109.63577139$

A4 $=109.90659661$

A5 $=110.51456687$

A6 $=110.78355215$

A7 $=112.3063092$

A8 $=111.70602969$

A9 $=107.97083233$

A10 $=124.07179534$

A11 $=127.83968927$

A12 $=69.41624722$

A13 $=163.17203913$

A14 $=47.88077202$

A15 $=107.10019345$

A16 $=117.27298056$

A17 $=111.00723404$

D1 $=166.91328575$

$\mathrm{D} 2=49.14094382$

D3 $=-71.88277767$

D4=173.18558629

D5 $=53.82062817$

D6 $=-68.28526091$

D7 $=45.20367958$ 

D8 $=73.76077205$
D9 $=-50.13552252$
D10 $=45.31872366$
D11 $=52.38762872$
D12 $=-135.96423638$
D13 $=-123.35069255$
D14 $=2.53289461$
D15 $=133.18393318$
D16=-123.57201916

\section{$\mathrm{HOCH}_{2} \mathrm{CH}_{2} \mathrm{CH}_{2} \mathrm{CHO} \cdot \cdot \mathrm{HNO}_{3}$ (TS7A)}

Final structure in terms of initial Z-matrix:

C

$\mathrm{H}, 1, \mathrm{~B} 1$

$\mathrm{C}, 1, \mathrm{~B} 2,2, \mathrm{~A} 1$

$\mathrm{H}, 3, \mathrm{~B} 3,1, \mathrm{~A} 2,2, \mathrm{D} 1,0$

H,3,B4,1,A3,2,D2,0

$\mathrm{C}, 3, \mathrm{~B} 5,1, \mathrm{~A} 4,2, \mathrm{D} 3,0$

H,6,B6,3,A5,1,D4,0

H,6,B7,3,A6,1,D5,0

C,6,B8,3,A7,1,D6,0

O,1,B9,3,A8,6,D7,0

H,10,B10,1,A9,3,D8,0

O,9,B11,6,A10,3,D9,0

N,12,B12,9,A11,6,D10,0

O,13,B13,12,A12,9,D11,0

O,13,B14,12,A13,9,D12,0

O,13,B15,12,A14,9,D13,0

H,12,B16,9,A15,6,D14,0

H,9,B17,6,A16,3,D15,0

H,1,B18,10,A17,9,D16,0

Variables:

B1 $=1.08853313$

B2 $=1.5191377$

B3 $=1.08811626$

B4 $=1.08763504$

B5 $=1.53089575$

B6 $=1.08856002$

B7 $=1.08940854$

B8 $=1.5165845$

B9 $=1.43044191$

B10 $=0.99286583$

$\mathrm{B} 11=1.26433224$

B12 $=3.11635522$

$\mathrm{B} 13=1.24564356$ 


$$
\begin{aligned}
& B 14=1.19698179 \\
& B 15=1.28838386 \\
& B 16=1.12900967 \\
& B 17=1.087816 \\
& B 18=1.0881361 \\
& A 1=111.88419359 \\
& A 2=110.16013013 \\
& A 3=111.86561636 \\
& A 4=105.13873241 \\
& A 5=111.64122808 \\
& A 6=110.0127536 \\
& A 7=110.38160945 \\
& A 8=106.36605889 \\
& A 9=106.26404142 \\
& \text { A10 }=122.28369255 \\
& A 11=109.24115375 \\
& A 12=72.86159433 \\
& A 13=163.36645363 \\
& A 14=46.04806031 \\
& A 15=114.66649322 \\
& A 16=117.11113545 \\
& A 17=109.19766782 \\
& D 1=166.73837304 \\
& D 2=46.32514999 \\
& D 3=-74.74578302 \\
& D 4=-161.75236254 \\
& D 5=77.53025447 \\
& D 6=-41.8202454 \\
& D 7=41.36656414 \\
& D 8=76.29720957 \\
& D 9=-87.70931362 \\
& D 10=59.16302651 \\
& D 11=63.12911465 \\
& D 12=-136.83355645 \\
& D 13=-107.82612475 \\
& D 14=34.24347479 \\
& D 15=120.26780255 \\
& D 16=-147.6325067
\end{aligned}
$$

\section{$\underline{\mathrm{HOCH}_{2} \mathrm{CH}_{2} \mathrm{CH}_{2} \mathrm{CHO} \bullet \cdot \mathrm{HNO}_{3}(\mathrm{PC} 7 \mathrm{~A})}$}

Final structure in terms of initial Z-matrix:

C

H,1,B1

$\mathrm{C}, 1, \mathrm{~B} 2,2, \mathrm{~A} 1$

H,3,B3,1,A2,2,D1,0 


$$
\begin{aligned}
& \text { H,3,B4,1,A3,2,D2,0 } \\
& \text { C,3,B5,1,A4,2,D3,0 } \\
& \text { H,6,B6,3,A5,1,D4,0 } \\
& \text { H,6,B7,3,A6,1,D5,0 } \\
& \text { C,6,B8,3,A7,1,D6,0 } \\
& \text { O,9,B9,6,A8,3,D7,0 } \\
& \text { H,10,B10,9, A9,6,D8,0 } \\
& \text { O,9,B11,6,A10,3,D9,0 } \\
& \text { N,10,B12,9,A11,6,D10,0 } \\
& \text { O,13,B13,10,A12,9,D11,0 } \\
& \text { O,13,B14,10,A13,9,D12,0 } \\
& \text { O,13,B15,10,A14,9,D13,0 } \\
& \text { H,12,B16,9,A15,6,D14,0 } \\
& \text { H,9,B17,6,A16,3,D15,0 } \\
& \text { H,1,B18,10,A17,9,D16,0 } \\
& \text { Variables: } \\
& \text { B1 }=1.09062942 \\
& \text { B2 }=1.52042471 \\
& \text { B3 }=1.08921976 \\
& \text { B4 }=1.08639123 \\
& \text { B5 }=1.52805189 \\
& \text { B6 }=1.08583773 \\
& \text { B7 }=1.09010866 \\
& \mathrm{~B} 8=1.52567605 \\
& \text { B9=1.43714295 } \\
& \mathrm{B} 10=1.58226796 \\
& \text { B11 }=1.3854421 \\
& \text { B12 }=3.2589088 \\
& \mathrm{~B} 13=1.33828646 \\
& \text { B14 }=1.18797201 \\
& \mathrm{~B} 15=1.21752804 \\
& \text { B16 }=0.96556941 \\
& \text { B17 }=1.0869567 \\
& \text { B18 }=1.08829388 \\
& \text { A1 }=111.77920638 \\
& \text { A2 }=110.14484104 \\
& \text { A3 }=112.36913577 \\
& \text { A4 }=101.09383621 \\
& \text { A5 }=114.66488965 \\
& \text { A6 }=110.22103661 \\
& \text { A7 }=102.51623239 \\
& \text { A8 }=104.46222462 \\
& \text { A9 }=112.8927954 \\
& \text { A10 }=114.55403457 \\
& \text { A11 }=105.63005858 \\
& \text { A12 }=49.13984432
\end{aligned}
$$



A13 $=164.82608048$
A14 $=68.08268893$
A15 $=109.28131586$
A16 $=113.82991893$
A17 $=108.18283742$
D1 $=157.86571439$
D2 $=36.63210193$
D3 $=-84.55303435$
D4 $=-159.09090523$
D5 $=77.55819956$
D6 $=-38.95205471$
$\mathrm{D} 7=31.66438366$
D8 $=-134.32357897$
D9 $=-88.78280672$
D10 $=-111.50198475$
D11=-118.80561681
D12 $=-129.2566408$
$\mathrm{D} 13=58.77899722$
D14 $=58.61627762$
D15 $=148.80900683$
D16=-135.61551365

\section{$\underline{\text { 2-OH-THF••HNO }}$ (RC8)}

Final structure in terms of initial Z-matrix:

C

$\mathrm{H}, 1, \mathrm{~B} 1$

$\mathrm{C}, 1, \mathrm{~B} 2,2, \mathrm{~A} 1$

$\mathrm{H}, 3, \mathrm{~B} 3,1, \mathrm{~A} 2,2, \mathrm{D} 1,0$

H,3,B4,1,A3,2,D2,0

C,3,B5,1,A4,2,D3,0

H,6,B6,3,A5,1,D4,0

H,6,B7,3,A6,1,D5,0

$\mathrm{C}, 6, \mathrm{~B} 8,3, \mathrm{~A} 7,1, \mathrm{D} 6,0$

O,9,B9,6,A8,3,D7,0

O,9,B10,6,A9,3,D8,0

H,9,B11,6,A10,3,D9,0

H,1,B12,10,A11,9,D10,0

H,11,B13,9,A12,6,D11,0

N,11,B14,9,A13,6,D12,0

O,15,B15,11,A14,9,D13,0

O,15,B16,11,A15,9,D14,0

O,15,B17,11,A16,9,D15,0

H,18,B18,15,A17,11,D16,0

Variables:

B1=1.0857991 


\begin{tabular}{|c|}
\hline $32=1.52628409$ \\
\hline$B 3=1.08582791$ \\
\hline $\mathrm{B} 4=1.08783857$ \\
\hline $\mathrm{B} 5=1.54040587$ \\
\hline$B 6=1.08708388$ \\
\hline B7 $=1.086451$ \\
\hline$B 8=1.53187541$ \\
\hline B9=1.38589953 \\
\hline $\mathrm{B} 10=1.43013999$ \\
\hline $\mathrm{B} 11=1.08871467$ \\
\hline $\mathrm{B} 12=1.09048144$ \\
\hline $\mathrm{B} 13=0.96195258$ \\
\hline B14 $=3.3769914$ \\
\hline B15 $=1.19005562$ \\
\hline $\mathrm{B} 16=1.20809593$ \\
\hline $\mathrm{B} 17=1.34914317$ \\
\hline B18 $=0.99814706$ \\
\hline $\mathrm{A} 1=114.23133791$ \\
\hline A2 $=111.88521523$ \\
\hline A3 $=110.11778994$ \\
\hline A4=103.04662006 \\
\hline $\mathrm{A} 5=112.41903173$ \\
\hline A6 $=112.00464314$ \\
\hline A7 $=103.79704285$ \\
\hline A8 $=107.34951587$ \\
\hline A9=108.32505771 \\
\hline $\mathrm{A} 10=114.83190378$ \\
\hline A11=109.8241458 \\
\hline $\mathrm{A} 12=108.24861314$ \\
\hline A13=115.3587194 \\
\hline A14=161.31168104 \\
\hline A15 $=70.73933511$ \\
\hline A16 $=46.0081236$ \\
\hline $\mathrm{A} 17=106.47024429$ \\
\hline D1=92.10717408 \\
\hline $\mathrm{D} 2=-29.12959785$ \\
\hline D3=-146.93054566 \\
\hline $\mathrm{D} 4=-107.60367382$ \\
\hline D5=130.58608406 \\
\hline D6=11.43796009 \\
\hline D7 $=11.44069863$ \\
\hline D8 $=-107.72293947$ \\
\hline D9=131.08833983 \\
\hline D10=-79.92006888 \\
\hline $\begin{array}{l}\mathrm{D} 11=166.91844206 \\
D 12=2295977375\end{array}$ \\
\hline
\end{tabular}


D13=-138.87176078

D14 $=41.98934057$

D15 $=-137.12288237$

D16 $=-0.38887617$

\section{$\underline{2-\mathrm{OH}-\mathrm{THF} \cdot \cdot \mathrm{HNO}_{3} \text { (TS8) }}$}

Final structure in terms of initial Z-matrix:

C

H,1,B1

C,1,B2,2,A1

H,3,B3,1,A2,2,D1,0

H,3,B4, 1,A3,2,D2,0

C,3,B5,1,A4,2,D3,0

H,6,B6,3,A5,1,D4,0

H,6,B7,3,A6,1,D5,0

C,6,B8,3,A7,1,D6,0

O,9,B9,6,A8,3,D7,0

O,9,B10,6,A9,3,D8,0

H,9,B11,6,A10,3,D9,0

H,1,B12,10,A11,9,D10,0

H,11,B13,9,A12,6,D11,0

N,11,B14,9,A13,6,D12,0

O,15,B15,11,A14,9,D13,0

O,15,B16,11,A15,9,D14,0

O,15,B17,11,A16,9,D15,0

H,11,B18,9,A17,6,D16,0

Variables:

B1=1.08332404

B2 $=1.52339536$

B3 $=1.08592937$

B4 $=1.08895637$

$\mathrm{B} 5=1.53381925$

B6 $=1.22789999$

$\mathrm{B} 7=1.08170286$

$\mathrm{B} 8=1.40730341$

B9 $=1.27942356$

$\mathrm{B} 10=2.57266421$

B11 $=1.0796401$

$\mathrm{B} 12=1.08817737$

$\mathrm{B} 13=0.95810386$

B14 $=3.31957412$

$\mathrm{B} 15=1.20385435$

$\mathrm{B} 16=1.28336545$

$\mathrm{B} 17=1.24315933$

B18 $=0.97871758$ 

A1=116.31949851
$\mathrm{A} 2=111.10929616$
A3 $=111.89921106$
A4 $=100.01848876$
A5 $=100.82600233$
A6 $=121.8800874$
A7 $=103.77023428$
A8 $=114.79398501$
A9=104.36852395
A10 $=129.01721932$
A11 $=105.63982493$
A12 $=134.24774019$
A13 $=87.59270498$
A14=151.20462545
A15 $=73.66135896$
A16 $=51.60497448$
A17 $=99.53727601$
D1 $=96.97226018$
D2 $=-26.04636384$
D3 $=-144.86244112$
D4 $=-78.48973793$
D5 $=162.98158224$
D6 $=26.51539805$
D7 $=-16.34307721$
D8 $=-127.02963592$
D9 $=164.81470048$
D10=-97.77343555
D11=114.20675983
D12 $=13.21485077$
D13 $=159.26194433$
D14 $=37.13289086$
D15 $=-110.78399775$
D16=-8.59309955

\section{$\underline{\text { 2-OH-THF } \bullet \mathrm{HNO}_{3} \text { (PC8) }}$}

Final structure in terms of initial Z-matrix:

C

$\mathrm{H}, 1, \mathrm{~B} 1$

$\mathrm{C}, 1, \mathrm{~B} 2,2, \mathrm{~A} 1$

H,3,B3,1,A2,2,D1,0

H,3,B4,1,A3,2,D2,0

C,3,B5,1,A4,2,D3,0

H,1,B6,3,A5,6,D4,0

H,6,B7,3,A6,1,D5,0

C,6,B8,3,A7,1,D6,0 


$$
\begin{aligned}
& \text { O,9,B9,6,A8,3,D7,0 } \\
& \text { O,10,B10,9,A9,6,D8,0 } \\
& \text { H,9,B11,6,A10,3,D9,0 } \\
& \text { H,1,B12,10,A11,9,D10,0 } \\
& \text { H,11,B13,10,A12,9,D11,0 } \\
& \text { N,11,B14,10,A13,9,D12,0 } \\
& \text { O,15,B15,11,A14,10,D13,0 } \\
& \text { O,15,B16,11,A15,10,D14,0 } \\
& \text { O,15,B17,11,A16,10,D15,0 } \\
& \text { H,11,B18,10,A17,9,D16,0 } \\
& \text { Variables: } \\
& \text { B1 }=1.08881979 \\
& \text { B2 }=1.53973542 \\
& \text { B3 }=1.08994117 \\
& \text { B4 }=1.08839709 \\
& \text { B5 }=1.50858875 \\
& \text { B6 }=3.25925362 \\
& \text { B7 }=1.07607557 \\
& \text { B8 }=1.32002064 \\
& \text { B9 }=1.37549413 \\
& \text { B10 }=2.73981498 \\
& \text { B11 }=1.07796009 \\
& \text { B12 }=1.08805229 \\
& \text { B13 }=0.97504595 \\
& \text { B14 }=3.19178467 \\
& \text { B15 }=1.19328436 \\
& \text { B16 }=1.34206583 \\
& \text { B17 }=1.21054135 \\
& \text { B18 }=0.96169717 \\
& \text { A1 }=111.8080876 \\
& \text { A2 }=112.6904091 \\
& \text { A3 }=111.18807285 \\
& \text { A4 }=101.24350726 \\
& \text { A5 }=88.01362671 \\
& \text { A6 }=125.35650649 \\
& \text { A7 }=109.24035684 \\
& \text { A8 }=114.64844017 \\
& \text { A9 }=105.59954894 \\
& \text { A10 }=130.58462667 \\
& \text { A11 }=107.83090834 \\
& \text { A12 }=15.55230092 \\
& \text { A13 }=82.5180958 \\
& \text { A14=162.98354684 } \\
& \text { A15 }=50.98198205 \\
& \text { A16 }=67.1049361 \\
& \text { A17 }=113.74414998 \\
&
\end{aligned}
$$



D1 $=136.0324454$
$\mathrm{D} 2=14.84827182$
D3 $=-105.05196968$
D4 $=76.91652573$
D5 $=174.47400552$
D6 $=-7.92549987$
D7 $=1.08488252$
D8 $=-109.23698591$
D9 $=-177.80686453$
D10 $=-134.57142688$
D11 $=-124.0653077$
D12 $=93.75968867$
D13 $=-42.62034767$
D14 $=-83.53719101$
D15 $=107.68357422$
D16 $=171.740748$

\section{$\underline{\text { trans }-\mathrm{H}_{2} \mathrm{SO}_{4}}$}

Final structure in terms of initial Z-matrix:

$\mathrm{S}$

$\mathrm{O}, 1, \mathrm{~B} 1$

$\mathrm{O}, 1, \mathrm{~B} 2,2, \mathrm{~A} 1$

O,1,B3,2,A2,3,D1,0

$\mathrm{O}, 1, \mathrm{~B} 4,4, \mathrm{~A} 3,2, \mathrm{D} 2,0$

H,2,B5,1,A4,4,D3,0

H,5,B6,1,A5,4,D4,0

Variables:

B1 $=1.57461883$

B2 $=1.57477984$

B3 $=1.41262207$

B4 $=1.4127153$

B5 $=0.96637276$

B6 $=3.20212809$

A1 $=102.0775399$

A2 $=105.97082931$

A3 $=123.74050339$

A4 $=109.57879923$

A5 $=30.27474451$

D1 $=113.57116224$

D2 $=-126.05103381$

D3 $=-161.04006172$

D4=-115.88689092 


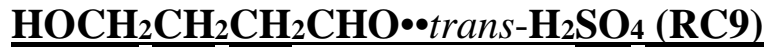

Final structure in terms of initial Z-matrix:

C

$\mathrm{H}, 1, \mathrm{~B} 1$

$\mathrm{C}, 1, \mathrm{~B} 2,2, \mathrm{~A} 1$

$\mathrm{H}, 3, \mathrm{~B} 3,1, \mathrm{~A} 2,2, \mathrm{D} 1,0$

$\mathrm{H}, 3, \mathrm{~B} 4,1, \mathrm{~A} 3,2, \mathrm{D} 2,0$

C,3,B5,1,A4,2,D3,0

H,6,B6,3,A5,1,D4,0

H,6,B7,3,A6,1,D5,0

C,6,B8,3,A7,1,D6,0

O,1,B9,3,A8,6,D7,0

H,10,B10,1,A9,3,D8,0

O,9,B11,6,A10,3,D9,0

H,9,B12,6,A11,3,D10,0

H,1,B13,10,A12,3,D11,0

S,12,B14,9,A13,6,D12,0

O,15,B15,12,A14,9,D13,0

O,15,B16,12,A15,9,D14,0

O,15,B17,12,A16,9,D15,0

O,15,B18,12,A17,9,D16,0

H,16,B19,15,A18,12,D17,0

H,17,B20,15,A19,12,D18,0

Variables:

B1=1.09146751

B2 $=1.51948554$

B3 $=1.08922703$

B4=1.09229141

B5 $=1.5280559$

B6 $=1.09052313$

B7 $=1.09522138$

$\mathrm{B} 8=1.49519753$

B9=1.41617396

$\mathrm{B} 10=0.96385601$

B11 $=1.2154882$

$\mathrm{B} 12=1.09764665$

B13 $=1.09257844$

B14 $=3.35485728$

B15=1.57017325

$\mathrm{B} 16=1.53168614$

B17 $=1.42496283$

$\mathrm{B} 18=1.42491304$

$\mathrm{B} 19=0.96562421$

B20 $=1.03726699$

A1 $=110.44081234$ 


$$
\begin{aligned}
& \mathrm{A} 2=110.46762163 \\
& \text { A3 }=109.09410822 \\
& \text { A4 }=110.14556163 \\
& \text { A5 }=111.45165744 \\
& \text { A6 }=109.58248691 \\
& \text { A7 }=112.29632145 \\
& \text { A8 }=108.18839154 \\
& \text { A9 }=108.41568794 \\
& \mathrm{~A} 10=125.02797614 \\
& \text { A11 }=116.29405001 \\
& \text { A12 }=110.51480517 \\
& \text { A13 }=102.90937687 \\
& \text { A14 }=135.18461804 \\
& \text { A15 }=44.55494436 \\
& \text { A16 }=72.40380939 \\
& \text { A17 }=110.92752159 \\
& \text { A18 }=109.51512767 \\
& \text { A19=111.97399689 } \\
& \text { D1 }=60.19579089 \\
& \text { D2 }=-57.86775593 \\
& \text { D3 }=-178.42221706 \\
& \text { D4 }=-60.29207253 \\
& \text { D5 }=-179.39523902 \\
& \text { D6 }=64.19585139 \\
& \text { D7 }=-58.58693119 \\
& \text { D8 }=123.82627196 \\
& \text { D9 }=-136.79211344 \\
& \text { D10 }=47.84126459 \\
& \text { D11 }=-120.32552015 \\
& \text { D12 }=38.45866674 \\
& \text { D13 }=176.71914381 \\
& \text { D14 }=-132.41668858 \\
& \text { D15 }=80.97318138 \\
& \text { D16 }=-34.28953001 \\
& \text { D17 }=123.50475019 \\
& \text { D18 }=-0.07439111
\end{aligned}
$$

$\underline{\mathrm{HOCH}_{2} \mathrm{CH}_{2} \mathrm{CH}_{2} \mathrm{CHO} \bullet \text { trans- } \mathrm{H}_{2} \mathrm{SO}_{4} \text { (TS9) }}$

Final structure in terms of initial Z-matrix:
$\mathrm{C}$
$\mathrm{H}, 1, \mathrm{~B} 1$
$\mathrm{C}, 1, \mathrm{~B} 2,2, \mathrm{~A} 1$
H,3,B3,1,A2,2,D1,0
H,3,B4,1,A3,2,D2,0
C,3,B5,1,A4,2,D3,0 


\author{
H,6,B6,3,A5,1,D4,0 \\ H,6,B7,3,A6,1,D5,0 \\ C,6,B8,3,A7,1,D6,0 \\ O,1,B9,3,A8,6,D7,0 \\ H,10,B10,1,A9,3,D8,0 \\ O,9,B11,6,A10,3,D9,0 \\ H,9,B12,6,A11,3,D10,0 \\ H,1,B13,10,A12,9,D11,0 \\ S,12,B14,9,A13,6,D12,0 \\ O,15,B15,12,A14,9,D13,0 \\ O,15,B16,12,A15,9,D14,0 \\ O,15,B17,12,A16,9,D15,0 \\ O,15,B18,12,A17,9,D16,0 \\ H,16,B19,15,A18,12,D17,0 \\ H,12,B20,9,A19,6,D18,0 \\ Variables: \\ B1=1.08864424 \\ B2 $=1.52172907$ \\ B3 $=1.08726114$ \\ B4 $=1.09146219$ \\ $\mathrm{B} 5=1.52438442$ \\ B6 $=1.08952936$ \\ B7 $=1.09341988$ \\ B8 $=1.49447723$ \\ B9 $=1.42675204$ \\ B10 $=0.98018049$ \\ $\mathrm{B} 11=1.25255687$ \\ B12 $=1.08856762$ \\ $\mathrm{B} 13=1.09024276$ \\ B14 $=3.26377507$ \\ $\mathrm{B} 15=1.58139841$ \\ $\mathrm{B} 16=1.48382382$ \\ $\mathrm{B} 17=1.44288382$ \\ $\mathrm{B} 18=1.43392708$ \\ B19 $=0.96461288$ \\ $\mathrm{B} 20=1.10374331$ \\ A1 $=112.06301556$ \\ A2 $=111.41901943$ \\ A3 $=109.96363068$ \\ A4 $=106.0520575$ \\ A5 $=111.59367068$ \\ A6 $=111.14773404$ \\ A7 $=109.1644574$ \\ A8 $=105.79512115$ \\ A9 $=107.85532082$ \\ A10 $=124.37414424$
}



A11=117.33992438
A12 $=109.58650528$
A13 $=98.25897364$
A14 $=135.44545135$
A15 $=43.68478665$
A16 $=72.8332557$
A17 $=113.4933904$
A18 $=108.80693021$
A19 $=114.2624857$
D1 $=74.31016087$
D2 $=-45.51220651$
D3 $=-164.56501458$
D4 $=-67.93528226$
D5 $=170.91775674$
D6 $=54.6020885$
D7 $=-45.62770452$
D8 $=127.10535074$
D9 $=-144.05162816$
D10 $=55.87390506$
D11 $=-97.55762226$
D12 $=47.83330607$
D13 $=175.43928489$
D14 $=-127.23738869$
D15 $=82.4657825$
D16 $=-29.62240944$
D17 $=123.24236178$
D18 $=23.75703695$

$\underline{\mathrm{HOCH}_{2} \mathrm{CH}_{2} \mathrm{CH}_{2} \mathrm{CHO} \bullet \text { trans }-\mathrm{H}_{2} \mathrm{SO}_{4} \text { (PC9) }}$

Final structure in terms of initial Z-matrix:

C

$\mathrm{H}, 1, \mathrm{~B} 1$

$\mathrm{C}, 1, \mathrm{~B} 2,2, \mathrm{~A} 1$

$\mathrm{H}, 3, \mathrm{~B} 3,1, \mathrm{~A} 2,2, \mathrm{D} 1,0$

H,3,B4,1,A3,2,D2,0

$\mathrm{C}, 3, \mathrm{~B} 5,1, \mathrm{~A} 4,2, \mathrm{D} 3,0$

H,6,B6,3,A5,1,D4,0

H,6,B7,3,A6,1,D5,0

C,6,B8,3,A7,1,D6,0

O,1,B9,3,A8,6,D7,0

H,10,B10,1,A9,3,D8,0

O,9,B11,6,A10,3,D9,0

H,9,B12,6,A11,3,D10,0

H,1,B13,10,A12,9,D11,0

S, 10,B14,1,A13,3,D12,0 
O,15,B15,10,A14,1,D13,0

O,15,B16,10,A15,1,D14,0

O,15,B17,10,A16,1,D15,0

O,15,B18,10,A17,1,D16,0

H,16,B19,15,A18,10,D17,0

H,12,B20,9,A19,6,D18,0

Variables:

B1 $=1.08781735$

B2 $=1.53513742$

B3 $=1.08605541$

B4 $=1.08918153$

B5 $=1.52466993$

B6 $=1.09139753$

$\mathrm{B} 7=1.08651047$

$B 8=1.52008003$

B9 $=1.43910146$

B10 $=1.4764095$

$\mathrm{B} 11=1.36568499$

B12 $=1.09105117$

$\mathrm{B} 13=1.08882269$

B14=3.26227909

B15 $=1.56657412$

$\mathrm{B} 16=1.42603468$

B17 $=1.53497764$

B18 $=1.42527445$

$\mathrm{B} 19=0.966112$

$\mathrm{B} 20=0.9690109$

A1 $=112.78536358$

A2 $=111.64878266$

A3 $=110.43477635$

A4 $=103.02595698$

A5 $=111.1156132$

A6 $=113.80134633$

A7 $=102.49815684$

A8 $=106.2181503$

A9 $=111.01884437$

A10 $=117.54613071$

A11 $=112.09047021$

A12 $=107.71994236$

A13 $=96.45430564$

A14 $=148.38081345$

A15 $=89.13557487$

A16 $=47.89571531$

A17 $=85.95322948$

A18 $=109.75628258$

A19 $=110.42278873$ 

D1 $=100.34486032$
D2 $=-20.24131974$
D3 $=-138.1253357$
D4 $=-80.86440893$
D5 $=155.69900054$
D6 $=35.04548422$
$\mathrm{D} 7=-19.30926511$
D8 $=122.22507034$
D9 $=-158.98369606$
D10 $=76.29494848$
D11 $=-124.8671154$
D12 $=94.82828838$
D13 $=116.3207092$
D14 $=-123.36074013$
D15 $=117.76078306$
$\mathrm{D} 16=-4.58014412$
$\mathrm{D} 17=-81.93386652$
D18 $=42.12763817$

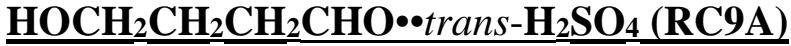

Final structure in terms of initial Z-matrix:

C

$\mathrm{H}, 1, \mathrm{~B} 1$

$\mathrm{C}, 1, \mathrm{~B} 2,2, \mathrm{~A} 1$

H,3,B3,1,A2,2,D1,0

H,3,B4,1,A3,2,D2,0

C,3,B5,1,A4,2,D3,0

H,6,B6,3,A5,1,D4,0

H,6,B7,3,A6,1,D5,0

C,6,B8,3,A7,1,D6,0

O,1,B9,3,A8,6,D7,0

H,10,B10,1,A9,3,D8,0

O,9,B11,6,A10,3,D9,0

H,9,B12,6,A11,3,D10,0

H,1,B13,10,A12,3,D11,0

S,12,B14,9,A13,6,D12,0

O,15,B15,12,A14,9,D13,0

O,15,B16,12,A15,9,D14,0

O,15,B17,12,A16,9,D15,0

O,15,B18,12,A17,9,D16,0

H,16,B19,15,A18,12,D17,0

H,17,B20,15,A19,12,D18,0

Variables:

B1 $=1.09094682$

B2 $=1.52312678$ 
B3 $=1.09076375$

B4 $=1.08986356$

B5 $=1.53615376$

B6 $=1.09085223$

B7 $=1.08914038$

B8 $=1.49910431$

B9 $=1.41200565$

B10 $=0.9674371$

B11 $=1.21471095$

B12 $=1.09678021$

B13 $=1.09210391$

B14=3.32163575

B15 $=1.58393069$

B16 $=1.53646683$

B17 $=1.42557729$

B18 $=1.41450272$

$\mathrm{B} 19=0.96568199$

B20 $=1.01877989$

A1 $=109.60359265$

A2 $=110.51981789$

A3 $=110.00835345$

A4 $=109.76203931$

A5 $=109.96004945$

A6 $=110.84436587$

A7 $=109.44043244$

A8 $=111.25496185$

A9 $=110.56449885$

A10 $=120.83068$

A11 $=119.00139669$

A12 $=111.29789084$

A13 $=106.31967601$

A14 $=91.25448767$

A15 $=47.06056745$

A16 $=69.15548514$

A17 $=152.42832708$

A18 $=109.32523334$

A19 $=110.93588098$

D1 $=169.69587133$

$\mathrm{D} 2=50.70724118$

D3 $=-70.58553932$

D4 $=172.59650695$

D5 $=51.88148562$

D6 $=-69.84533225$

D7 $=46.50556136$

D8 $=79.21003579$

D9=-63.58814623 

$\mathrm{D} 10=117.06663302$
D11=-123.81139569
D12 $=133.15103359$
$\mathrm{D} 13=22.72763101$
D14 $=129.3483227$
D15 $=-81.9961233$
D16 $=159.56789357$
D17 $=129.24005357$
D18=-1.22103136

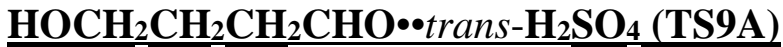

Final structure in terms of initial Z-matrix:

C

$\mathrm{H}, 1, \mathrm{~B} 1$

$\mathrm{C}, 1, \mathrm{~B} 2,2, \mathrm{~A} 1$

H,3,B3,1,A2,2,D1,0

$\mathrm{H}, 3, \mathrm{~B} 4,1, \mathrm{~A} 3,2, \mathrm{D} 2,0$

$\mathrm{C}, 3, \mathrm{~B} 5,1, \mathrm{~A} 4,2, \mathrm{D} 3,0$

H,6,B6,3,A5,1,D4,0

H,6,B7,3,A6,1,D5,0

C,6,B8,3,A7,1,D6,0

O,1,B9,3,A8,6,D7,0

H,10,B10,1,A9,3,D8,0

O,9,B11,6,A10,3,D9,0

H,9,B12,6,A11,3,D10,0

H,1,B13,10,A12,9,D11,0

S,12,B14,9,A13,6,D12,0

O,15,B15,12,A14,9,D13,0

O,15,B16,12,A15,9,D14,0

O,15,B17,12,A16,9,D15,0

O,15,B18,12,A17,9,D16,0

H,16,B19,15,A18,12,D17,0

H,12,B20,9,A19,6,D18,0

Variables:

B1=1.08961796

B2 $=1.52834104$

B3 $=1.08854421$

B4 $=1.08763745$

B5 $=1.53174072$

$\mathrm{B} 6=1.0894757$

$\mathrm{B} 7=1.08854583$

$\mathrm{B} 8=1.49664162$

B9=1.42342335

$\mathrm{B} 10=0.98942253$

$\mathrm{B} 11=1.25151418$ 


$$
\begin{aligned}
& \text { B12 }=1.09031145 \\
& \text { B13 }=1.08831194 \\
& \text { B14 }=3.26597354 \\
& \text { B15 }=1.59474547 \\
& \text { B16 }=1.49057197 \\
& \mathrm{~B} 17=1.44485972 \\
& \text { B18 }=1.42025529 \\
& \text { B19 }=0.96455046 \\
& \text { B20 }=1.14933421 \\
& \text { A1 }=110.5790607 \\
& \text { A2 }=110.79169568 \\
& \text { A3 }=111.36627072 \\
& \text { A4 }=105.64260085 \\
& \text { A5 }=111.49009462 \\
& \text { A6 }=111.26549771 \\
& \mathrm{~A} 7=106.54367501 \\
& \text { A8 }=107.67305758 \\
& \text { A9 }=113.12383878 \\
& \text { A10 }=117.96736189 \\
& \text { A11 }=120.85970191 \\
& \text { A12 }=110.39139032 \\
& \text { A13 }=102.3654557 \\
& \text { A14 }=106.21044535 \\
& \text { A15 }=42.53809379 \\
& \text { A16 }=68.66247617 \\
& \text { A17 }=142.59633955 \\
& \text { A18 }=108.6679125 \\
& \text { A19 }=112.70082356 \\
& \text { D1 }=157.56008538 \\
& \text { D2 }=36.67355237 \\
& \text { D3 }=-84.55450506 \\
& \text { D4 }=-173.75822687 \\
& \text { D5 }=63.87406454 \\
& \text { D6 }=-55.94274643 \\
& \text { D7 }=32.10402433 \\
& \text { D8 }=105.47855955 \\
& \text { D9 }=-64.30656925 \\
& \mathrm{D} 10=132.85440368 \\
& \text { D11 }=-126.018415 \\
& \text { D12 }=148.44439457 \\
& \text { D13 }=20.06086719 \\
& \text { D14 }=113.13793285 \\
& \text { D15=-77.98344647 } \\
& \text { D16 }=173.14485985 \\
& \text { D17 }=131.50403101 \\
& \text { D18 }=175.17582847
\end{aligned}
$$




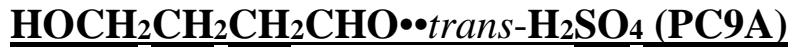

Final structure in terms of initial Z-matrix:

C

$\mathrm{H}, 1, \mathrm{~B} 1$

$\mathrm{C}, 1, \mathrm{~B} 2,2, \mathrm{~A} 1$

H,3,B3,1,A2,2,D1,0

$\mathrm{H}, 3, \mathrm{~B} 4,1, \mathrm{~A} 3,2, \mathrm{D} 2,0$

C,3,B5,1,A4,2,D3,0

H,6,B6,3,A5,1,D4,0

H,6,B7,3,A6,1,D5,0

C,6,B8,3,A7,1,D6,0

O,9,B9,6,A8,3,D7,0

H,10,B10,9,A9,6,D8,0

O,9,B11,6,A10,3,D9,0

H,9,B12,6,A11,3,D10,0

H,1,B13,10,A12,9,D11,0

S,10,B14,9,A13,6,D12,0

O,15,B15,10,A14,9,D13,0

O,15,B16,10,A15,9,D14,0

O,15,B17,10,A16,9,D15,0

O,15,B18,10,A17,9,D16,0

H,16,B19,15,A18,10,D17,0

H,12,B20,9,A19,6,D18,0

Variables:

B1=1.08847877

B2 $=1.53115048$

B3 $=1.08685155$

B4 $=1.08627314$

B5 $=1.53090306$

B6 $=1.08533457$

$\mathrm{B} 7=1.08885482$

$\mathrm{B} 8=1.51498213$

B9 $=1.44078163$

B10 $=1.49062$

$\mathrm{B} 11=1.38032526$

B12 $=1.0893918$

B13 $=1.08841724$

B14=3.39944667

B15 $=1.58070444$

$\mathrm{B} 16=1.42666972$

$\mathrm{B} 17=1.53381699$

$\mathrm{B} 18=1.41490876$

$\mathrm{B} 19=0.9656796$

B20 $=0.96676608$

A1=112.53736256 


$$
\begin{aligned}
& \text { A2 }=110.4651126 \\
& \text { A3 }=111.89701356 \\
& \text { A4 }=102.74899866 \\
& \text { A5 }=113.58195168 \\
& \text { A6 }=111.16887051 \\
& \text { A7 }=101.44134252 \\
& \text { A8 }=102.91858866 \\
& \text { A9 }=116.99079795 \\
& \text { A10 }=109.25112028 \\
& \text { A11 }=115.69657863 \\
& \text { A12 }=109.07593295 \\
& \text { A13 }=95.15511278 \\
& \text { A14 }=91.81562746 \\
& \text { A15 }=72.29027103 \\
& \text { A16 }=43.55148272 \\
& \text { A17 }=149.89901977 \\
& \text { A18 }=109.25499105 \\
& \text { A19 }=108.15874161 \\
& \text { D1 }=145.15300882 \\
& \text { D2 }=23.68184612 \\
& \text { D3 }=-97.85939188 \\
& \text { D4 }=-155.77372887 \\
& \text { D5 }=80.00720177 \\
& \text { D6 }=-36.25971345 \\
& \text { D7 }=41.04626095 \\
& \text { D8 }=-166.82012309 \\
& \text { D9=-77.06959933 } \\
& \text { D10 }=155.48619471 \\
& \text { D11=-114.26709036 } \\
& \text { D12 }=-179.0436479 \\
& \text { D13=-99.55118697 } \\
& \text { D14 }=4.81083807 \\
& \text { D15 }=152.81495618 \\
& \text { D16 }=127.26922049 \\
& \text { D17 }=-126.03595597 \\
& \text { D18 }=-171.90730174
\end{aligned}
$$

\section{$\underline{\text { 2-OH-THF} \bullet \bullet t r a n s-H_{2} \mathrm{SO}_{4}(\mathrm{RC10})}$}

Final structure in terms of initial Z-matrix:
$\mathrm{C}$
$\mathrm{H}, 1, \mathrm{~B} 1$
C,1,B2,2,A1
H,3,B3,1,A2,2,D1,0
H,3,B4,1,A3,2,D2,0
C,3,B5,1,A4,2,D3,0 
H,6,B6,3,A5,1,D4,0

H,6,B7,3,A6,1,D5,0

C,6,B8,3,A7,1,D6,0

O,9,B9,6,A8,3,D7,0

O,9,B10,6,A9,3,D8,0

H,9,B11,6,A10,3,D9,0

H,1,B12,10,A11,9,D10,0

H,11,B13,9,A12,6,D11,0

S,11,B14,9,A13,6,D12,0

O,15,B15,11,A14,9,D13,0

O,15,B16,11,A15,9,D14,0

O,15,B17,11,A16,9,D15,0

O,15,B18,11,A17,9,D16,0

H,16,B19,15,A18,11,D17,0

H,17,B20,15,A19,11,D18,0

Variables:

B1=1.09036855

$\mathrm{B} 2=1.52833683$

B3 $=1.08824445$

B4=1.08632649

B5 $=1.53062231$

B6 $=1.08647289$

B7 $=1.08932258$

$\mathrm{B} 8=1.51494324$

B9 $=1.38953866$

$\mathrm{B} 10=1.43065613$

B11 $=1.08859871$

B12 $=1.08791905$

B13 $=0.96184215$

B14 $=3.53126693$

$\mathrm{B} 15=1.54654876$

B16 $=1.58748647$

$\mathrm{B} 17=1.41608912$

$\mathrm{B} 18=1.41717237$

$\mathrm{B} 19=1.00464377$

B20 $=0.96541958$

A1 $=111.90229191$

A2 $=110.97412907$

A3 $=112.41973158$

A4 $=101.93300709$

A5 $=114.84417306$

A6 $=110.77439267$

A7 $=100.9521462$

A8 $=106.58856701$

A9 $=108.21714009$

A10 $=115.36714269$ 

A11 $=108.8274478$
$\mathrm{A} 12=108.58947088$
A13 $=114.00806806$
A14 $=41.60879272$
A15 $=99.02992171$
A16=143.95017397
A17 $=70.77228244$
$\mathrm{A} 18=112.28392716$
A19=108.90460589
$\mathrm{D} 1=150.17031461$
D2 $=28.77761502$
D3 $=-92.37884607$
D4 $=-156.74391428$
D5 $=79.33610865$
D6 $=-35.38850624$
$\mathrm{D} 7=35.3873121$
D8 $=-82.98202722$
D9 $=155.68797107$
D10 $=-125.19730876$
D11 $=163.30348367$
D12=-7.3746971
D13 $=131.62519047$
D14=33.18820235
$\mathrm{D} 15=170.77809263$
D16 $=-69.83918176$
D17 $=-0.76301035$
D18=129.40316882

\section{$\underline{\text { 2-OH-THF } \bullet \text { trans- } \mathrm{H}_{2} \mathrm{SO}_{4} \text { (TS10) }}$}

Final structure in terms of initial Z-matrix:

C

$\mathrm{H}, 1, \mathrm{~B} 1$

$\mathrm{C}, 1, \mathrm{~B} 2,2, \mathrm{~A} 1$

$\mathrm{H}, 3, \mathrm{~B} 3,1, \mathrm{~A} 2,2, \mathrm{D} 1,0$

H,3,B4,1,A3,2,D2,0

C,3,B5,1,A4,2,D3,0

H,6,B6,3,A5,1,D4,0

H,6,B7,3,A6,1,D5,0

C,6,B8,3,A7,1,D6,0

O,9,B9,6,A8,3,D7,0

O,1,B10,10,A9,9,D8,0

H,9,B11,6,A10,3,D9,0

H,1,B12,10,A11,9,D10,0

H,11,B13,1,A12,10,D11,0

S,6,B14,3,A13,1,D12,0 
O,15,B15,6,A14,3,D13,0

O,15,B16,6,A15,3,D14,0

O,15,B17,6,A16,3,D15,0

O,15,B18,6,A17,3,D16,0

H,11,B19,1,A18,10,D17,0

H,17,B20,15,A19,6,D18,0

Variables:

B1 $=1.08429782$

$\mathrm{B} 2=1.53260477$

B3 $=1.08685561$

B4 $=1.08869582$

B5 $=1.52989437$

B6 $=1.29142165$

$\mathrm{B} 7=1.08266809$

B8 $=1.39240844$

B9 $=1.28290648$

B10 $=4.47591904$

$\mathrm{B} 11=1.08207322$

B12 $=1.08649922$

B13 $=0.9581685$

B14 $=3.44872992$

$\mathrm{B} 15=1.44277468$

B16 $=1.6058836$

$\mathrm{B} 17=1.42344254$

B18 $=1.4841735$

$\mathrm{B} 19=0.96959849$

B20 $=0.9643567$

A1 $=115.36331084$

A2 $=110.66528991$

A3 $=111.93370865$

A4 $=101.34391827$

A5 $=105.23200111$

A6 $=122.22321061$

A7 $=105.01560681$

A8 $=115.57234685$

A9 $=35.30287368$

A10 $=128.43251292$

A11 $=105.53694546$

A12 $=151.71255353$

A13 $=87.64141776$

A14 $=72.2477112$

A15 $=89.76538354$

A16 $=156.37020308$

A17 $=45.16687582$

A18 $=54.29757661$

A19 $=108.24810035$ 


$$
\begin{aligned}
& \text { D1 }=104.98120576 \\
& \text { D2 }=-17.02590784 \\
& \text { D3 }=-135.95529428 \\
& \text { D4 }=-89.25711304 \\
& \text { D5 }=156.93460555 \\
& \text { D6 }=18.17649049 \\
& \text { D7 }=-11.17928219 \\
& \text { D8 }=-59.46454574 \\
& \text { D9 }=172.87861246 \\
& \text { D10 }=-105.14587454 \\
& \text { D11 }=166.59957352 \\
& \text { D12 }=-73.13635314 \\
& \text { D13 }=92.2033086 \\
& \text { D14 }=-11.02393751 \\
& \text { D15 }=-146.1590265 \\
& \text { D16 }=-121.60003771 \\
& \text { D17 }=-143.50062244 \\
& \text { D18 }=-135.56154372
\end{aligned}
$$

\section{$\underline{\text { 2-OH-THF••trans-H2 }} \mathrm{SO}_{4}$ (PC10)}

Final structure in terms of initial Z-matrix:

C

$\mathrm{H}, 1, \mathrm{~B} 1$

$\mathrm{C}, 1, \mathrm{~B} 2,2, \mathrm{~A} 1$

$\mathrm{H}, 3, \mathrm{~B} 3,1, \mathrm{~A} 2,2, \mathrm{D} 1,0$

H,3,B4,1,A3,2,D2,0

C,3,B5,1,A4,2,D3,0

H,6,B6,3,A5,1,D4,0

H,6,B7,3,A6,1,D5,0

$\mathrm{C}, 6, \mathrm{~B} 8,3, \mathrm{~A} 7,1, \mathrm{D} 6,0$

O,9,B9,6,A8,3,D7,0

O,10,B10,9,A9,6,D8,0

H,9,B11,6,A10,3,D9,0

H,1,B12,10,A11,9,D10,0

H,11,B13,10,A12,9,D11,0

S,11,B14,10,A13,9,D12,0

O,15,B15,11,A14,10,D13,0

$\mathrm{O}, 15, \mathrm{~B} 16,11, \mathrm{~A} 15,10, \mathrm{D} 14,0$

O,15,B17,11,A16,10,D15,0

O,15,B18,11,A17,10,D16,0

H,11,B19,10,A18,9,D17,0

H,17,B20,15,A19,11,D18,0

Variables:

B1 $=1.08915288$

B2 $=1.5377685$ 


$$
\begin{aligned}
& \text { B3=1.08949787 } \\
& \text { B4 }=1.08875967 \\
& \text { B5 }=1.50786581 \\
& \text { B6 }=3.97657323 \\
& \mathrm{~B} 7=1.0759534 \\
& \text { B8 }=1.31943784 \\
& \text { B9 }=1.37597442 \\
& \text { B10 }=2.68792647 \\
& \text { B11 }=1.0784964 \\
& \text { B12 }=1.08476704 \\
& \text { B13 }=0.98087925 \\
& \text { B14 }=3.37868491 \\
& \text { B15 }=1.42368988 \\
& \text { B16 }=1.58338901 \\
& \text { B17 }=1.41867464 \\
& \text { B18 }=1.53510777 \\
& \mathrm{~B} 19=0.96010128 \\
& \text { B20 }=0.96523294 \\
& \text { A1 }=111.89862837 \\
& \text { A2 }=111.90486662 \\
& \text { A3 }=111.4307378 \\
& \text { A4 }=101.42489534 \\
& \text { A5 }=96.93295381 \\
& \text { A6 }=125.45611564 \\
& \text { A7 }=109.18701419 \\
& \text { A8 }=114.57242494 \\
& \text { A9=96.16772204 } \\
& \text { A10 }=130.68897414 \\
& \text { A11 }=107.77038933 \\
& \text { A12 }=12.25914564 \\
& \text { A13 }=82.1534743 \\
& \text { A14 }=64.66665957 \\
& \text { A15 }=113.84696245 \\
& \text { A16 }=134.99660699 \\
& \text { A17 }=44.60631055 \\
& \text { A18 }=119.07303052 \\
& \text { A19 }=108.60579954 \\
& \text { D1 }=136.89042394 \\
& \text { D2 }=15.29117171 \\
& \text { D3 }=-104.78561464 \\
& \text { D4 }=-59.25896195 \\
& \text { D5 }=175.24878316 \\
& \text { D6 }=-7.96723338 \\
& \text { D7 }=1.21708103 \\
& \text { D8 }=-127.63681804 \\
& \text { D9 }=-177.26779136
\end{aligned}
$$



$\mathrm{D} 10=-133.28668946$
$\mathrm{D} 11=172.29691376$
D12 $=69.89319115$
$\mathrm{D} 13=47.83173961$
D14 $=-48.89322972$
D15 $=157.66942876$
D16 $=-133.01242455$
$\mathrm{D} 17=171.43938492$
D18=-136.69672274

\section{$\underline{\text { cis- }-\mathrm{H}_{2} \mathrm{SO}_{4}}$}

Final structure in terms of initial Z-matrix:

$\mathrm{S}$

$\mathrm{O}, 1, \mathrm{~B} 1$

$\mathrm{O}, 1, \mathrm{~B} 2,2, \mathrm{~A} 1$

O,1,B3,2,A2,3,D1,0

H,4,B4,1,A3,2,D2,0

$\mathrm{O}, 1, \mathrm{~B} 5,2, \mathrm{~A} 4,4, \mathrm{D} 3,0$

H,6,B6,1,A5,2,D4,0

Variables:

B1 $=1.40562072$

$\mathrm{B} 2=1.42164118$

B3 $=1.57485055$

B4 $=0.9654652$

$\mathrm{B} 5=1.57364437$

B6 $=0.96561765$

$\mathrm{A} 1=123.63475781$

A2 $=106.74441373$

A3 $=109.63745606$

A4=106.55205926

A5 $=109.76110632$

D1 $=125.7137127$

D2 $=-149.00549519$

D3 $=108.49837209$

D4 $=151.98175527$

$\underline{\mathrm{HOCH}_{2} \mathrm{CH}_{2} \mathrm{CH}_{2} \mathrm{CHO} \cdot{ }^{\circ} \text { cis- } \mathrm{H}_{2} \mathrm{SO}_{4} \text { (TS9B) }}$

Final structure in terms of initial Z-matrix:

C

$\mathrm{H}, 1, \mathrm{~B} 1$

$\mathrm{C}, 1, \mathrm{~B} 2,2, \mathrm{~A} 1$

H,3,B3,1,A2,2,D1,0

H,3,B4,1,A3,2,D2,0

C,3,B5,1,A4,2,D3,0 


\author{
H,6,B6,3,A5,1,D4,0 \\ H,6,B7,3,A6,1,D5,0 \\ C,6,B8,3,A7,1,D6,0 \\ O,1,B9,3,A8,6,D7,0 \\ H,10,B10,1,A9,3,D8,0 \\ O,9,B11,6,A10,3,D9,0 \\ H,9,B12,6,A11,3,D10,0 \\ H,1,B13,10,A12,9,D11,0 \\ S,12,B14,9,A13,6,D12,0 \\ O,15,B15,12,A14,9,D13,0 \\ O,15,B16,12,A15,9,D14,0 \\ O,15,B17,12,A16,9,D15,0 \\ O,15,B18,12,A17,9,D16,0 \\ H,16,B19,15,A18,12,D17,0 \\ H,12,B20,9,A19,6,D18,0 \\ Variables: \\ B1=1.08865909 \\ B2 $=1.5219589$ \\ B3 $=1.08725225$ \\ B4 $=1.09150992$ \\ $\mathrm{B} 5=1.52459024$ \\ B6 $=1.08942613$ \\ B7 $=1.09350244$ \\ B8 $=1.49406453$ \\ $\mathrm{B} 9=1.4270368$ \\ B10 $=0.98059034$ \\ B11 $=1.25226123$ \\ B12 $=1.0886852$ \\ $\mathrm{B} 13=1.08998458$ \\ B14 $=3.26008128$ \\ $\mathrm{B} 15=1.579245$ \\ $\mathrm{B} 16=1.48659586$ \\ $\mathrm{B} 17=1.45068936$ \\ $\mathrm{B} 18=1.42544318$ \\ B19 $=0.96516948$ \\ B20 $=1.1086396$ \\ A1 $=111.99956809$ \\ A2 $=111.39445756$ \\ A3 $=109.95793263$ \\ A4 $=106.16961717$ \\ A5 $=111.59961367$ \\ A6 $=111.08902245$ \\ A7 $=109.17455956$ \\ A8 $=105.84436802$ \\ A9 $=107.89376949$ \\ A10 $=124.4320863$
}



A11 $=117.2929653$
A12 $=109.59298456$
$\mathrm{A} 13=98.09773604$
A14=138.400125
A15 $=43.79491995$
A16 $=72.98002223$
A17 $=111.9866054$
A18 $=109.50444121$
A19 $=114.51123882$
D1 $=74.57362791$
D2 $=-45.20549117$
D3 $=-164.27844589$
D4 $=-68.03300244$
D5 $=170.87007463$
D6 $=54.62900327$
D7 $=-45.37073921$
D8 $=126.8412492$
D9 $=-144.10233599$
D10 $=55.66408831$
D11 $=-97.93471349$
D12 $=47.87356636$
D13 $=-179.91325966$
D14 $=-129.54340368$
$\mathrm{D} 15=83.53953748$
D16 $=-28.54582354$
D17 $=-31.21204867$
$\mathrm{D} 18=24.11128776$

$\underline{\mathrm{HOCH}} \underline{\mathrm{CH}}_{2} \underline{\mathrm{CH}}_{2} \mathrm{CHO} \cdot{ }^{\mathrm{C} i s}-\mathrm{H}_{2} \mathrm{SO}_{4}$ (RC9B)

Final structure in terms of initial Z-matrix:

C

$\mathrm{H}, 1, \mathrm{~B} 1$

$\mathrm{C}, 1, \mathrm{~B} 2,2, \mathrm{~A} 1$

H,3,B3,1,A2,2,D1,0

H,3,B4,1,A3,2,D2,0

C,3,B5,1,A4,2,D3,0

H,6,B6,3,A5,1,D4,0

H,6,B7,3,A6,1,D5,0

C,6,B8,3,A7,1,D6,0

O,1,B9,3,A8,6,D7,0

H,10,B10,1,A9,3,D8,0

O,9,B11,6,A10,3,D9,0

H,9,B12,6,A11,3,D10,0

H,1,B13,10,A12,3,D11,0

S,12,B14,9,A13,6,D12,0 
O,15,B15,12,A14,9,D13,0

O,15,B16,12,A15,9,D14,0

O,15,B17,12,A16,9,D15,0

O,15,B18,12,A17,9,D16,0

H,16,B19,15,A18,12,D17,0

H,17,B20,15,A19,12,D18,0

Variables:

B1 $=1.09143012$

B2 $=1.51947891$

B3 $=1.08920628$

B4 $=1.09233765$

$\mathrm{B} 5=1.52823821$

B6 $=1.09052786$

B7 $=1.09526344$

B8 $=1.49464947$

B9 $=1.41680176$

B10 $=0.96406519$

$\mathrm{B} 11=1.21566178$

B12 $=1.09774475$

B13 $=1.09229929$

B14 $=3.35377315$

$\mathrm{B} 15=1.56835228$

$\mathrm{B} 16=1.53491641$

$\mathrm{B} 17=1.43236957$

$\mathrm{B} 18=1.41667305$

$\mathrm{B} 19=0.96629608$

$\mathrm{B} 20=1.03611434$

A1 $=110.39221568$

A2 $=110.4388912$

A3 $=109.12191468$

A4 $=110.19078467$

A5 $=111.46912295$

A6 $=109.56359651$

A7 $=112.22127192$

A8 $=108.23862179$

A9 $=108.44721479$

A10 $=125.06572389$

A11 $=116.28523947$

A12 $=110.54479723$

A13 $=102.917642$

A14 $=137.90178186$

A15 $=44.76594133$

A16 $=72.82162844$

A17 $=109.29366671$

A18 $=110.03128897$

A19 $=111.62852557$ 

D1 $=60.51282911$
D2 $=-57.54285018$
D3 $=-178.11947408$
D4=-60.14820939
D5 $=-179.29700751$
D6 $=64.34464605$
$\mathrm{D} 7=-58.31702929$
D8 $=123.67673717$
D9 $=-136.7050099$
D10 $=47.87233956$
D11 $=-120.3593934$
$\mathrm{D} 12=38.72340243$
D13 $=-178.05105803$
D14 $=-134.09995949$
$\mathrm{D} 15=82.65424153$
$\mathrm{D} 16=-32.8016208$
$\mathrm{D} 17=-41.67421886$
D18 $=0.60703809$

$\underline{\mathrm{HOCH}}_{2} \mathrm{CH}_{2} \mathrm{CH}_{2} \mathrm{CHO} \cdot \bullet^{\circ i s-\mathrm{H}_{2} \mathrm{SO}_{4}}$ (PC9B)

Final structure in terms of initial Z-matrix:

C

$\mathrm{H}, 1, \mathrm{~B} 1$

$\mathrm{C}, 1, \mathrm{~B} 2,2, \mathrm{~A} 1$

$\mathrm{H}, 3, \mathrm{~B} 3,1, \mathrm{~A} 2,2, \mathrm{D} 1,0$

H,3,B4,1,A3,2,D2,0

C,3,B5,1,A4,2,D3,0

H,6,B6,3,A5,1,D4,0

H,6,B7,3,A6,1,D5,0

C,6,B8,3,A7,1,D6,0

O,1,B9,3,A8,6,D7,0

H,10,B10,1,A9,3,D8,0

O,9,B11,6,A10,3,D9,0

H,9,B12,6,A11,3,D10,0

H,1,B13,10,A12,9,D11,0

S,10,B14,1,A13,3,D12,0

O,15,B15,10,A14,1,D13,0

O,15,B16,10,A15,1,D14,0

O,15,B17,10,A16,1,D15,0

O,15,B18,10,A17,1,D16,0

H,16,B19,15,A18,10,D17,0

H,12,B20,9,A19,6,D18,0

Variables:

B1 $=1.08766958$

B2 $=1.53486714$ 


$$
\begin{aligned}
& \text { B3=1.08601577 } \\
& \text { B4=1.08918035 } \\
& \text { B5 }=1.52465326 \\
& \text { B6 }=1.09149763 \\
& \text { B7 }=1.08650841 \\
& B 8=1.51994716 \\
& \text { B9 }=1.43997168 \\
& \mathrm{~B} 10=1.47325823 \\
& \text { B11 }=1.36655696 \\
& \text { B12 }=1.09098408 \\
& \mathrm{~B} 13=1.08885942 \\
& \text { B14 }=3.26315021 \\
& \text { B15 }=1.56626184 \\
& \text { B16 }=1.43405657 \\
& \text { B17 }=1.53532586 \\
& \text { B18 }=1.41754948 \\
& \mathrm{~B} 19=0.96634637 \\
& \text { B20 }=0.96917035 \\
& \text { A1 }=112.82889264 \\
& \mathrm{~A} 2=111.63572713 \\
& \mathrm{~A} 3=110.44202463 \\
& \text { A4 }=103.05748303 \\
& \text { A5 }=111.08156074 \\
& \text { A6 }=113.80495437 \\
& \mathrm{~A} 7=102.52350091 \\
& \text { A8 }=106.18682209 \\
& \text { A9 }=110.44829497 \\
& \text { A10 }=117.43617054 \\
& \text { A11 }=112.12501638 \\
& \text { A12 }=107.70680274 \\
& \text { A13 }=95.93466052 \\
& \text { A14 }=147.99802327 \\
& \text { A15 }=89.29084236 \\
& \text { A16 }=47.75075485 \\
& \text { A17 }=85.99055284 \\
& \text { A18 }=109.9299497 \\
& \text { A19 }=110.25286479 \\
& \text { D1 }=100.30663733 \\
& \text { D2 }=-20.29264321 \\
& \text { D3 }=-138.15981855 \\
& \text { D4 }=-80.96860733 \\
& \text { D5 }=155.64158156 \\
& \text { D6 }=34.98376893 \\
& \mathrm{D} 7=-19.38536563 \\
& \text { D8 }=122.11573722 \\
& \text { D9 }=-158.75005275
\end{aligned}
$$



D10 $=76.57527944$
D11 $=-124.68669276$
D12 $=94.81010851$
D13 $=108.86357268$
D14 $=-125.77832185$
D15 $=116.72480653$
D16 $=-7.09518973$
$\mathrm{D} 17=82.79982413$
$\mathrm{D} 18=42.10152521$

\section{$\bullet$}

Final structure in terms of initial Z-matrix:

$\mathrm{O}$

H,1,B1

Variables:

B1 $=0.97080278$

\section{$\underline{\mathbf{H}_{2}} \underline{\mathrm{O}}$}

Final structure in terms of initial Z-matrix:

$\mathrm{H}$

$\mathrm{O}, 1, \mathrm{~B} 1$

$\mathrm{H}, 2, \mathrm{~B} 2,1, \mathrm{~A} 1$

Variables:

$\mathrm{B} 1=0.95852857$

B2 $=0.95858951$

A1=105.08576069

\section{$\underline{\text { 2-OH-THF} \bullet O H ~(T S 11) ~}$}

Final structure in terms of initial Z-matrix:

C

$\mathrm{H}, 1, \mathrm{~B} 1$

$\mathrm{C}, 1, \mathrm{~B} 2,2, \mathrm{~A} 1$

H,3,B3,1,A2,2,D1,0

H,3,B4,1,A3,2,D2,0

C,3,B5,1,A4,2,D3,0

H,6,B6,3,A5,1,D4,0

H,6,B7,3,A6,1,D5,0

C,6,B8,3,A7,1,D6,0

O,9,B9,6,A8,3,D7,0

O,9,B10,6,A9,3,D8,0

H,9,B11,6,A10,3,D9,0 
H,1,B12,10,A11,9,D10,0

H,11,B13,9,A12,6,D11,0

O,1,B14,10,A13,9,D12,0

H,15,B15,1,A14,10,D13,0

Variables:

B1=1.09130724

B2 $=1.52695719$

B3 $=1.08816278$

B4 $=1.08656896$

B5 $=1.5311723$

B6 $=1.08508377$

B7 $=1.08902738$

B8 $=1.51633491$

B9 $=1.4005899$

B10 $=1.384971$

B11 $=1.1513176$

B12 $=1.08763321$

B13 $=0.96223481$

B14 $=4.17920737$

B15 $=0.97079139$

A1 $=111.57187779$

A2 $=110.48577085$

A3 $=112.39765768$

A4 $=101.68281106$

A5 $=114.45049294$

A6 $=110.92851252$

A7 $=100.76777358$

A8 $=106.63590431$

A9 $=110.09002022$

A10 $=113.53148873$

A11 $=108.63885857$

A12 $=108.34541307$

A13 $=21.20958822$

A14 $=53.88016047$

D1 $=154.41700346$

$\mathrm{D} 2=32.47010314$

D3 $=-89.04459288$

D4 $=-156.72634685$

D5 $=78.70612734$

D6 $=-37.05115604$

D7 $=34.1813192$

D8 $=-87.8957113$

D9=149.92276099

D10=-129.65613032

D11 $=174.16619648$

D12 $=67.97749467$ 
D13=78.7143939

\section{2-OH-THF••OH (RC11)}

Final structure in terms of initial Z-matrix:

C

$\mathrm{H}, 1, \mathrm{~B} 1$

$\mathrm{C}, 1, \mathrm{~B} 2,2, \mathrm{~A} 1$

H,3,B3,1,A2,2,D1,0

H,3,B4,1,A3,2,D2,0

C,3,B5,1,A4,2,D3,0

H,6,B6,3,A5,1,D4,0

H,6,B7,3,A6,1,D5,0

C,6,B8,3,A7,1,D6,0

O,9,B9,6,A8,3,D7,0

O,9,B10,6,A9,3,D8,0

H,9,B11,6,A10,3,D9,0

H,1,B12,10,A11,9,D10,0

H,11,B13,9,A12,6,D11,0

O,9,B14,6,A13,3,D12,0

H,15,B15,9,A14,6,D13,0

Variables:

B1=1.08981915

$\mathrm{B} 2=1.53061857$

B3 $=1.08720216$

B4 $=1.08647426$

$\mathrm{B} 5=1.53032572$

B6 $=1.08545762$

$\mathrm{B} 7=1.08911496$

$\mathrm{B} 8=1.51648368$

B9 $=1.42081125$

$\mathrm{B} 10=1.39395498$

B11 $=1.09083804$

$\mathrm{B} 12=1.08831701$

$\mathrm{B} 13=0.96520834$

B14=3.17699954

$\mathrm{B} 15=0.98301083$

$\mathrm{A} 1=112.06024167$

A2 $=110.54535899$

A3 $=112.08563372$

A4 $=102.29763979$

A5 $=113.86303667$

A6 $=111.04430399$

A7 $=101.13147807$

A8 $=104.52854058$

A9=108.44794261 

$\mathrm{A} 10=114.88478486$
A11 $=109.28770556$
$\mathrm{A} 12=108.42007881$
A13 $=158.68603408$
A14 $=44.11528245$
D1 $=147.90650509$
D2 $=26.30015295$
D3 $=-95.24035243$
D4 $=-155.97932713$
D5 $=79.76838531$
D6 $=-36.18818468$
$\mathrm{D} 7=38.74172503$
D8 $=-80.24280555$
D9 $=154.70323695$
D10 $=-119.3649376$
D11=176.00898757
D12=-3.94321516
$\mathrm{D} 13=53.52801835$

\section{$\underline{\text { 2-OH-THF••OH (PC11) }}$}

Final structure in terms of initial Z-matrix:

C

H,1,B1

$\mathrm{C}, 1, \mathrm{~B} 2,2, \mathrm{~A} 1$

H,3,B3,1,A2,2,D1,0

H,3,B4,1,A3,2,D2,0

C,3,B5,1,A4,2,D3,0

H,6,B6,3,A5,1,D4,0

H,6,B7,3,A6,1,D5,0

C,6,B8,3,A7,1,D6,0

O,9,B9,6,A8,3,D7,0

O,9,B10,6,A9,3,D8,0

H,9,B11,6,A10,3,D9,0

H,1,B12,10,A11,9,D10,0

H,11,B13,9,A12,6,D11,0

O,9,B14,6,A13,3,D12,0

H,15,B15,9,A14,6,D13,0

Variables:

B1=1.09310489

B2 $=1.52579399$

B3 $=1.08836601$

B4=1.0865609

B5 $=1.53778505$

B6 $=1.08912061$

B7 $=1.08938131$ 


$$
\begin{aligned}
& \mathrm{B} 8=1.50809633 \\
& \text { B9 }=1.39192784 \\
& \text { B10 }=1.34615189 \\
& \text { B11 }=3.61539248 \\
& \text { B12 }=1.08609606 \\
& \mathrm{~B} 13=0.97249587 \\
& \text { B14 }=3.14229772 \\
& \text { B15 }=0.96674827 \\
& \text { A1 }=111.30295546 \\
& \text { A2 }=109.48101628 \\
& \text { A3 }=112.56124383 \\
& \text { A4 }=102.09343245 \\
& \text { A5 }=113.1994619 \\
& \text { A6 }=111.43197564 \\
& \text { A7 }=102.93566624 \\
& \text { A8 }=109.64891565 \\
& \text { A9 }=117.57203034 \\
& \text { A10 }=172.55461684 \\
& \text { A11 }=107.85209872 \\
& \text { A12 }=108.73949281 \\
& \text { A13 }=169.97339898 \\
& \text { A14 }=53.27918205 \\
& \text { D1 }=161.05789943 \\
& \mathrm{D} 2=40.03798932 \\
& \text { D3 }=-82.12291823 \\
& \text { D4 }=-141.89192479 \\
& \text { D5=96.16150166 } \\
& \text { D6 }=-22.88330568 \\
& \text { D7 }=2.68891216 \\
& \mathrm{D} 8=-129.7024338 \\
& \text { D9=69.31952001 } \\
& \text { D10 }=-157.4526771 \\
& \text { D11 }=174.5229947 \\
& \text { D12 }=-38.85501982 \\
& \text { D13 }=56.17809972
\end{aligned}
$$

\section{2-OH-THF••OH (P11)}

Final structure in terms of initial Z-matrix:
C
$\mathrm{H}, 1, \mathrm{~B} 1$
$\mathrm{C}, 1, \mathrm{~B} 2,2, \mathrm{~A} 1$
H,3,B3,1,A2,2,D1,0
H,3,B4,1,A3,2,D2,0
C,3,B5,1,A4,2,D3,0
H,6,B6,3,A5,1,D4,0 


$$
\begin{aligned}
& \text { H,6,B7,3,A6,1,D5,0 } \\
& \text { C,6,B8,3,A7,1,D6,0 } \\
& \text { O,9,B9,6,A8,3,D7,0 } \\
& \text { O,9,B10,6,A9,3,D8,0 } \\
& \text { H,1,B11,10,A10,9,D9,0 } \\
& \text { H,11,B12,9,A11,6,D10,0 } \\
& \text { Variables: } \\
& \text { B1 }=1.09302238 \\
& \text { B2 }=1.52731255 \\
& \text { B3 }=1.08837238 \\
& \text { B4 }=1.08656056 \\
& \text { B5 }=1.53872579 \\
& \text { B6 }=1.08934462 \\
& \text { B7 }=1.08917075 \\
& \text { B8 }=1.50827175 \\
& \text { B9 }=1.37422774 \\
& \text { B10 }=1.35861452 \\
& \text { B11 }=1.08590845 \\
& \text { B12 }=0.96178819 \\
& \text { A1 }=111.33602251 \\
& \text { A2 }=109.53242209 \\
& \text { A3 }=112.6447184 \\
& \text { A4 }=102.05370163 \\
& \text { A5 }=113.23449434 \\
& \text { A6 }=111.57824004 \\
& \text { A7 }=102.47660901 \\
& \text { A8 }=110.463824 \\
& \text { A9 }=116.93960947 \\
& \text { A10 }=107.79093148 \\
& \text { A11 }=108.56033535 \\
& \text { D1 }=160.44865412 \\
& \text { D2 }=39.37927238 \\
& \text { D3 }=-82.75895958 \\
& \text { D4 }=-140.9613384 \\
& \text { D5 }=96.97984606 \\
& \text { D6 }=-21.89774057 \\
& \text { D7 }=1.61960547 \\
& \text { D8 }=-130.23208175 \\
& \text { D9 }=-157.55338229 \\
& \text { D10 }=171.48761901 \\
&
\end{aligned}
$$

\section{2-OH-THF••OH (TS12)}

Final structure in terms of initial Z-matrix:

C

H,1,B1 
$\mathrm{C}, 1, \mathrm{~B} 2,2, \mathrm{~A} 1$

H,3,B3,1,A2,2,D1,0

H,3,B4,1,A3,2,D2,0

C,3,B5,1,A4,2,D3,0

H,6,B6,3,A5,1,D4,0

H,6,B7,3,A6,1,D5,0

C,6,B8,3,A7,1,D6,0

O,9,B9,6,A8,3,D7,0

O,9,B10,6,A9,3,D8,0

H,9,B11,6,A10,3,D9,0

H,1,B12,10,A11,9,D10,0

H,11,B13,9,A12,6,D11,0

O,11,B14,9,A13,6,D12,0

H,15,B15,11,A14,9,D13,0

Variables:

B1 $=1.08744819$

B2 $=1.52537717$

B3 $=1.0868061$

B4 $=1.08996977$

B5 $=1.52347672$

B6 $=1.09005538$

B7 $=1.08609219$

$\mathrm{B} 8=1.52996665$

B9 $=1.41824981$

$\mathrm{B} 10=1.37376181$

$\mathrm{B} 11=1.09889583$

$\mathrm{B} 12=1.09408307$

$\mathrm{B} 13=1.08709948$

B14 $=2.28145006$

B15 $=0.97105398$

$\mathrm{A} 1=113.87617617$

A2 $=112.55843682$

A3 $=110.20076675$

A4 $=100.87236347$

A5 $=110.82621448$

A6 $=114.51153544$

A7 $=102.32395089$

A8 $=106.15138135$

A9 $=116.0816315$

A10 $=111.40518414$

A11 $=109.36111107$

A12 $=110.86868244$

A13 $=98.5908264$

A14 $=89.86025529$

$\mathrm{D} 1=85.41122282$

D2 $=-36.04020082$ 


$$
\begin{aligned}
& \text { D3 }=-153.15547253 \\
& \text { D4 }=-78.22831721 \\
& \text { D5 }=157.76699606 \\
& \text { D6 }=36.70224166 \\
& \text { D7 }=-27.30971717 \\
& \text { D8 }=-151.47077084 \\
& \text { D9 }=90.56861264 \\
& \text { D10 }=-100.51917222 \\
& \text { D11 }=60.5825801 \\
& \text { D12 }=67.72614372 \\
& \text { D13 }=63.86772423
\end{aligned}
$$

\section{2-OH-THF••OH (RC12)}

Final structure in terms of initial Z-matrix:

$$
\begin{aligned}
& \text { C } \\
& \text { H,1,B1 } \\
& \text { C,1,B2,2,A1 } \\
& \text { H,3,B3,1,A2,2,D1,0 } \\
& \text { H,3,B4,1,A3,2,D2,0 } \\
& \text { C,3,B5,1,A4,2,D3,0 } \\
& \text { H,6,B6,3,A5,1,D4,0 } \\
& \text { H,6,B7,3,A6,1,D5,0 } \\
& \text { C,6,B8,3,A7,1,D6,0 } \\
& \text { O,1,B9,3,A8,6,D7,0 } \\
& \text { O,9,B10,6,A9,3,D8,0 } \\
& \text { H,9,B11,6,A10,3,D9,0 } \\
& \text { H,1,B12,10,A11,9,D10,0 } \\
& \text { H,11,B13,9,A12,6,D11,0 } \\
& \text { O,11,B14,9,A13,6,D12,0 } \\
& \text { H,15,B15,11,A14,9,D13,0 } \\
& \text { Variables: } \\
& \text { B1 }=1.08765616 \\
& \text { B2=1.52453553 } \\
& \text { B3 }=1.086867 \\
& \text { B4=1.09001509 } \\
& \text { B5 }=1.52269227 \\
& \text { B6=1.09297238 } \\
& \text { B7=1.08631578 } \\
& \text { B8=1.52524315 } \\
& \text { B9=1.42880819 } \\
& \text { B10 }=1.38172324 \\
& \text { B11 }=1.09065637 \\
& \text { B12 }=1.09548942 \\
& \text { B13 }=0.96518502 \\
& \text { B14 }=2.9485816
\end{aligned}
$$




$$
\begin{aligned}
& \mathrm{B} 15=0.98166437 \\
& \mathrm{~A} 1=113.9314872 \\
& \mathrm{~A} 2=112.62485413 \\
& \mathrm{~A} 3=110.2544522 \\
& \mathrm{~A} 4=100.79840468 \\
& \mathrm{~A} 5=110.33970768 \\
& \mathrm{~A} 6=114.6794171 \\
& \mathrm{~A} 7=102.5919722 \\
& \mathrm{~A} 8=105.55271364 \\
& \mathrm{~A} 9=115.42260822 \\
& \mathrm{~A} 10=112.12732152 \\
& \mathrm{~A} 11=109.37400068 \\
& \mathrm{~A} 12=109.02153518 \\
& \mathrm{~A} 13=90.17175515 \\
& \mathrm{~A} 14=56.51728081 \\
& \mathrm{D} 1=85.71542169 \\
& \mathrm{D} 2=-35.77493086 \\
& \mathrm{D} 3=-152.60904784 \\
& \mathrm{D} 4=-76.80115555 \\
& \mathrm{D} 5=160.18438745 \\
& \mathrm{D} 6=38.55105397 \\
& \mathrm{D} 7=-33.7749589 \\
& \mathrm{D} 8=-152.60689361 \\
& \mathrm{D} 9=86.35356601 \\
& \mathrm{D} 10=-103.47071147 \\
& \mathrm{D} 11=54.63713865 \\
& \mathrm{D} 12=84.07948057 \\
& \mathrm{D} 13=51.22346592
\end{aligned}
$$

\section{2-OH-THF••OH (PC12)}

Final structure in terms of initial Z-matrix:

C

$\mathrm{H}, 1, \mathrm{~B} 1$

$\mathrm{C}, 1, \mathrm{~B} 2,2, \mathrm{~A} 1$

H,3,B3,1,A2,2,D1,0

$\mathrm{H}, 3, \mathrm{~B} 4,1, \mathrm{~A} 3,2, \mathrm{D} 2,0$

C,3,B5,1,A4,2,D3,0

H,6,B6,3,A5,1,D4,0

H,6,B7,3,A6,1,D5,0

C,6,B8,3,A7,1,D6,0

O,9,B9,6,A8,3,D7,0

O,9,B10,6,A9,3,D8,0

H,9,B11,6,A10,3,D9,0

H,1,B12,10,A11,9,D10,0

H,11,B13,9,A12,6,D11,0 

O,11,B14,9,A13,6,D12,0
H,15,B15,11,A14,9,D13,0
Variables:
B1=1.08744944
B2 $=1.52596431$
B3 $=1.08689791$
B4 $=1.09003714$
B5 $=1.52194249$
B6 $=1.08881673$
B7 $=1.08485071$
$\mathrm{B} 8=1.55292036$
$\mathrm{B} 9=1.41720651$
$\mathrm{B} 10=1.32886922$
B11 $=1.104961$
$\mathrm{B} 12=1.09136553$
$\mathrm{B} 13=2.85883542$
B14 $=2.95324159$
B15 $=0.96474687$
A1 $=113.53538364$
A2 $=112.33691884$
A3 $=110.52909951$
A4 $=101.72820305$
A5 $=111.07381442$
A6 $=115.30943112$
$\mathrm{A} 7=101.10636239$
A8 $=106.00152487$
A9 $=111.36577601$
A10 $=109.8468488$
A11 $=109.0802084$
A12 $=107.80047158$
A13 $=89.28046615$
A14 $=45.7622713$
D1 $=86.46599281$
D2 $=-35.31010412$
D3 $=-152.55132783$
D4=-76.40922819
D5 $=157.50673295$
D6 $=37.47633432$
$\mathrm{D} 7=-29.17646065$
D8 $=-153.6667911$
D9 $=87.93096198$
D10 $=-102.84061406$
D11 $=71.18042443$
D12 $=75.02251588$
D13 $=63.41887461$ 


\section{2-OH-THF••OH (P12)}

Final structure in terms of initial Z-matrix:

C

$\mathrm{H}, 1, \mathrm{~B} 1$

$\mathrm{C}, 1, \mathrm{~B} 2,2, \mathrm{~A} 1$

H,3,B3,1,A2,2,D1,0

$\mathrm{H}, 3, \mathrm{~B} 4,1, \mathrm{~A} 3,2, \mathrm{D} 2,0$

C,3,B5,1,A4,2,D3,0

H,6,B6,3,A5,1,D4,0

H,6,B7,3,A6,1,D5,0

$\mathrm{C}, 6, \mathrm{~B} 8,3, \mathrm{~A} 7,1, \mathrm{D} 6,0$

O,9,B9,6,A8,3,D7,0

O,9,B10,6,A9,3,D8,0

H,9,B11,6,A10,3,D9,0

H,1,B12,10,A11,9,D10,0

Variables:

B1 $=1.08626387$

B2 $=1.52576317$

B3 $=1.08719104$

B4 $=1.08841396$

$\mathrm{B} 5=1.53085623$

$\mathrm{B} 6=1.08786226$

B7 $=1.08512091$

$\mathrm{B} 8=1.573857$

B9 $=1.40323935$

$\mathrm{B} 10=1.34257028$

B11=1.0953589

B12=1.09468329

A1=114.36901879

A2 $=112.99218897$

A3 $=109.44193651$

A4 $=102.1912167$

A5 $=111.80930343$

A6 $=114.20622683$

A7 $=102.73668554$

A8 $=106.49529421$

A9 $=105.89860708$

A10 $=112.49987389$

A11=110.12910511

D1 $=82.1575052$

D2 $=-39.47044772$

D3 $=-156.59689322$

D4=-94.90892229

D5 $=141.28458633$

D6 $=21.9137573$ 


$$
\begin{aligned}
& \text { D7 }=1.61055568 \\
& \text { D8 }=-120.76209662 \\
& \text { D9 }=119.07472486 \\
& \text { D10 }=-76.72977467
\end{aligned}
$$

\section{2-OH-THF・०OH (TS13)}

Final structure in terms of initial Z-matrix:

C

$\mathrm{H}, 1, \mathrm{~B} 1$,

$\mathrm{C}, 1, \mathrm{~B} 2,2, \mathrm{~A} 1$

$\mathrm{H}, 3, \mathrm{~B} 3,1, \mathrm{~A} 2,2, \mathrm{D} 1,0$

H,3,B4,1,A3,2,D2,0

C,3,B5,1,A4,2,D3,0

H,6,B6,3,A5,1,D4,0

H,6,B7,3,A6,1,D5,0

C,6,B8,3,A7,1,D6,0

$\mathrm{O}, 9, \mathrm{~B} 9,6, \mathrm{~A} 8,3, \mathrm{D} 7,0$

O,9,B10,6,A9,3,D8,0

H,9,B11,6,A10,3,D9,0

H,1,B12,10,A11,9,D10,0

H,11,B13,9,A12,6,D11,0

O,6,B14,3,A13,1,D12,0

H,15,B15,6,A14,3,D13,0

Variables:

B1 $=1.08797603$

$\mathrm{B} 2=1.54046992$

B3 $=1.08874374$

B4 $=1.08669087$

$\mathrm{B} 5=1.51852186$

B6 $=1.08528258$

B7 $=1.18237489$

B8 $=1.51215180$

B9 $=1.40329934$

B10 $=1.40179254$

B11 $=1.09055253$

B12 $=1.08863706$

B13 $=0.96106708$

B14 $=2.49819619$

B15 $=0.97175541$

$\mathrm{A} 1=112.51927573$

A2 $=110.92266232$

A3 $=112.29061204$

A4 $=102.67061277$

A5 $=117.48051401$

A6 $=107.73684313$ 


$$
\begin{aligned}
& A 7=102.56226699 \\
& A 8=103.88421041 \\
& A 9=107.83668466 \\
& A 10=114.71961991 \\
& A 11=109.48900224 \\
& \text { A12 }=108.41861624 \\
& \text { A13 }=101.69229371 \\
& A 14=85.27618231 \\
& D 1=134.02188258 \\
& D 2=12.23280700 \\
& D 3=-108.66967592 \\
& D 4=-156.71056955 \\
& D 5=82.06562749 \\
& D 6=-28.52242017 \\
& D 7=39.78857275 \\
& \text { D8 }=-79.11397776 \\
& D 9=156.20811914 \\
& D 10=-104.29664354 \\
& D 11=173.52613200 \\
& D 12=69.62944496 \\
& D 13=-74.47006775
\end{aligned}
$$

\section{$\underline{\text { 2-OH-THF••OH (RC13) }}$}

Final structure in terms of initial Z-matrix:

$$
\begin{aligned}
& \text { C } \\
& \text { H,1,B1 } \\
& \text { C,1,B2,2,A1 } \\
& \text { H,3,B3,1,A2,2,D1,0 } \\
& \text { H,3,B4,1,A3,2,D2,0 } \\
& \text { C,3,B5,1,A4,2,D3,0 } \\
& \text { H,6,B6,3,A5,1,D4,0 } \\
& \text { H,6,B7,3,A6,1,D5,0 } \\
& \text { C,6,B8,3,A7,1,D6,0 } \\
& \text { O,9,B9,6,A8,3,D7,0 } \\
& \text { O,9,B10,6,A9,3,D8,0 } \\
& \text { H,9,B11,6,A10,3,D9,0 } \\
& \text { H,1,B12,10,A11,9,D10,0 } \\
& \text { H,11,B13,9,A12,6,D11,0 } \\
& \text { O,10,B14,9,A13,6,D12,0 } \\
& \text { H,15,B15,10,A14,9,D13,0 } \\
& \text { Variables: } \\
& \text { B1=1.08945436 } \\
& \text { B2=1.53474379 } \\
& \text { B3=1.08690767 } \\
& \text { B4=1.08615808 }
\end{aligned}
$$




$$
\begin{aligned}
& \text { B5 }=1.53168000 \\
& \text { B6 }=1.08562819 \\
& \text { B7 }=1.09029913 \\
& \text { B8 }=1.51597915 \\
& \text { B9 }=1.41307174 \\
& \text { B10 }=1.39869197 \\
& \text { B11 }=1.09099125 \\
& \text { B12 }=1.08786207 \\
& \text { B13 }=0.96066458 \\
& \text { B14 }=2.78606644 \\
& \text { B15 }=0.97944595 \\
& \text { A1 }=112.23577280 \\
& \text { A2 }=110.53513012 \\
& \text { A3 }=111.96278144 \\
& \text { A4 }=102.77357260 \\
& \text { A5 }=113.83891581 \\
& \text { A6 }=111.20440664 \\
& \text { A7 }=101.15531904 \\
& \text { A8 }=104.65582322 \\
& \text { A9 }=108.27808592 \\
& \text { A10 }=114.76515628 \\
& \text { A11 }=109.06440730 \\
& \text { A12 }=108.58813399 \\
& \text { A13 }=93.20683647 \\
& \text { A14 }=19.61347677 \\
& \text { D1 }=141.86705087 \\
& \text { D2 }=20.47068177 \\
& \text { D3 }=-100.85341868 \\
& \text { D4 }=-153.44369943 \\
& \text { D5 }=82.31764509 \\
& \text { D6 }=-33.15550940 \\
& \text { D7 }=39.82289184 \\
& \text { D8 }=-78.83644162 \\
& \text { D9 }=156.16867453 \\
& \text { D10 }=-112.64929797 \\
& \text { D11 }=173.28389926 \\
& \text { D12 }=69.78119178 \\
& \text { D13 }=134.18459119
\end{aligned}
$$

\section{2-OH-THF••OH (PC13)}

Final structure in terms of initial Z-matrix:

C

$\mathrm{H}, 1, \mathrm{~B} 1$

$\mathrm{C}, 1, \mathrm{~B} 2,2, \mathrm{~A} 1$

H,3,B3,1,A2,2,D1,0 


$$
\begin{aligned}
& \text { H,3,B4,1,A3,2,D2,0 } \\
& \text { C,3,B5,1,A4,2,D3,0 } \\
& \text { H,6,B6,3,A5,1,D4,0 } \\
& \text { H,1,B7,3,A6,6,D5,0 } \\
& \text { C,6,B8,3,A7,1,D6,0 } \\
& \text { O,9,B9,6,A8,3,D7,0 } \\
& \text { O,9,B10,6,A9,3,D8,0 } \\
& \text { H,9,B11,6,A10,3,D9,0 } \\
& \text { H,1,B12,10,A11,9,D10,0 } \\
& \text { H,1,B13,9,A12,6,D11,0 } \\
& \text { O,10,B14,9,A13,6,D12,0 } \\
& \text { H,15,B15,10,A14,9,D13,0 } \\
& \text { Variables } \\
& \text { B1 }=1.08617293 \\
& \text { B2 }=1.53322945 \\
& \text { B3 }=1.08983506 \\
& \text { B4=1.09434415 } \\
& \text { B5 }=1.48786589 \\
& \text { B6 }=1.07600227 \\
& \text { B7 }=3.94695197 \\
& \text { B8 }=1.48899246 \\
& \text { B9 }=1.41541782 \\
& \text { B10 }=1.40495919 \\
& \text { B11 }=1.09316756 \\
& \text { B12 }=1.09069238 \\
& \text { B13 }=0.96166298 \\
& \text { B14=2.82078278 } \\
& \text { B15 }=0.96691817 \\
& \text { A1 }=113.88768722 \\
& \text { A2 }=112.41880670 \\
& \text { A3 }=110.45067445 \\
& \text { A4 }=101.30233571 \\
& \text { A5 }=126.82653612 \\
& \text { A6 }=86.22105914 \\
& \text { A7 }=109.39295021 \\
& \text { A8 }=104.41416114 \\
& \text { A9 }=109.35501781 \\
& \text { A10 }=114.62381624 \\
& \text { A11 }=109.37852248 \\
& \text { A12=108.41718107 } \\
& \text { A13=101.66225771 } \\
& \text { A14=16.88445966 } \\
& \text { D1 }=93.82909948 \\
& \text { D2 }=-27.09421333 \\
& \text { D3 }=-144.89560263 \\
& \text { D4 }=-167.55324125 \\
&
\end{aligned}
$$




$$
\begin{aligned}
& D 5=-84.27590743 \\
& D 6=10.34923153 \\
& D 7=10.83468722 \\
& D 8=-108.58911299 \\
& D 9=127.03583498 \\
& D 10=-82.37894906 \\
& D 11=-179.51368802 \\
& D 12=65.58700495 \\
& D 13=111.47585645
\end{aligned}
$$

\section{2-OH-THF••OH (TS14)}

Final structure in terms of initial Z-matrix:

C

$\mathrm{H}, 1, \mathrm{~B} 1$

$\mathrm{C}, 1, \mathrm{~B} 2,2, \mathrm{~A} 1$

H,3,B3,1,A2,2,D1,0

H,3,B4,1,A3,2,D2,0

$\mathrm{C}, 3, \mathrm{~B} 5,1, \mathrm{~A} 4,2, \mathrm{D} 3,0$

H,6,B6,3,A5,1,D4,0

H,6,B7,3,A6,1,D5,0

C,6,B8,3,A7,1,D6,0

O,9,B9,6,A8,3,D7,0

O,9,B10,6,A9,3,D8,0

H,9,B11,6,A10,3,D9,0

H,1,B12,10,A11,9,D10,0

H,11,B13,9,A12,6,D11,0

O,6,B14,3,A13,1,D12,0

H,15,B15,6,A14,3,D13,0

Variables:

B1=1.08601739

$\mathrm{B} 2=1.52321046$

B3 $=1.08738401$

B4 $=1.0906209$

B5 $=1.52067897$

$\mathrm{B} 6=1.17833362$

B7 $=1.08585799$

$\mathrm{B} 8=1.53064251$

B9 $=1.40041887$

$\mathrm{B} 10=1.40994815$

$\mathrm{B} 11=1.09143676$

$\mathrm{B} 12=1.09321172$

$\mathrm{B} 13=0.96083788$

B14 $=2.52202129$

B15 $=0.97196034$

A1=114.54318193 


$$
\begin{aligned}
& \text { A2 }=113.24635865 \\
& \text { A3 }=110.05727381 \\
& \text { A4 }=101.00110956 \\
& \text { A5 }=106.86119679 \\
& \text { A6 }=116.57311445 \\
& \mathrm{~A} 7=104.80397497 \\
& \text { A8 }=106.3788616 \\
& \text { A9 }=109.09382402 \\
& \text { A10 }=113.39619849 \\
& \text { A11 }=110.25192039 \\
& \text { A12 }=108.0583507 \\
& \text { A13 }=102.22522812 \\
& \text { A14 }=89.88645 \\
& \text { D1 }=84.30279624 \\
& \text { D2 }=-38.03756867 \\
& \text { D3 }=-155.27633973 \\
& \text { D4=-90.51429885 } \\
& \text { D5 }=151.97249211 \\
& \text { D6 }=23.99648933 \\
& \text { D7 }=-1.89385897 \\
& \text { D8 }=-122.13772426 \\
& \text { D9 }=115.60857767 \\
& \text { D10 }=-79.10661982 \\
& \text { D11 }=175.25939736 \\
& \text { D12 }=-82.47421221 \\
& \text { D13 }=116.65816618
\end{aligned}
$$

\section{2-OH-THF••OH (RC14)}

Final structure in terms of initial Z-matrix:

C

$\mathrm{H}, 1, \mathrm{~B} 1$

$\mathrm{C}, 1, \mathrm{~B} 2,2, \mathrm{~A} 1$

H,3,B3,1,A2,2,D1,0

H,3,B4,1,A3,2,D2,0

$\mathrm{C}, 3, \mathrm{~B} 5,1, \mathrm{~A} 4,2, \mathrm{D} 3,0$

H,6,B6,3,A5,1,D4,0

H,6,B7,3,A6,1,D5,0

C,6,B8,3,A7,1,D6,0

O,9,B9,6,A8,3,D7,0

O,9,B10,6,A9,3,D8,0

H,9,B11,6,A10,3,D9,0

H,1,B12,10,A11,9,D10,0

H,11,B13,9,A12,6,D11,0

O,11,B14,9,A13,6,D12,0

H,15,B15,11,A14,9,D13,0 


\footnotetext{
Variables:

B1=1.08608467

B2 $=1.53096617$

B3 $=1.08601119$

B4 $=1.08743842$

$\mathrm{B} 5=1.54281996$

B6 $=1.08664731$

B7 $=1.08649811$

$\mathrm{B} 8=1.52978473$

B9 $=1.39248388$

$\mathrm{B} 10=1.42142049$

$\mathrm{B} 11=1.08919874$

B12 $=1.09379829$

B13 $=0.96188344$

$\mathrm{B} 14=2.82115715$

B15 $=0.98015006$

A1 $=114.12856082$

A2 $=111.61407351$

$\mathrm{A} 3=110.30822842$

A4=103.35048786

A5 $=112.74385143$

A6 $=111.56046036$

A7 $=103.89102073$

A8 $=106.6985984$

A9=107.9984591

A10 $=114.78833827$

A11 $=109.95008872$

A12 $=107.92370864$

A13 $=107.01630387$

A14 $=11.33648851$

D1 $=96.09530742$

D2 $=-25.0117697$

D3 $=-143.18906002$

D4 $=-113.96053238$

D5 $=123.82830895$

D6 $=5.29536704$

D7=17.36239513

D8 $=-101.70888342$

D9=136.11126351

D10 $=-81.17853962$

D11 $=171.4366802$

D12 $=41.46279243$

D13=-163.41494492
}

$\underline{\text { 2-OH-THF••OH (PC14) }}$ 
Final structure in terms of initial Z-matrix:

C

$\mathrm{H}, 1, \mathrm{~B} 1$

$\mathrm{C}, 1, \mathrm{~B} 2,2, \mathrm{~A} 1$

$\mathrm{H}, 3, \mathrm{~B} 3,1, \mathrm{~A} 2,2, \mathrm{D} 1,0$

H,3,B4,1,A3,2,D2,0

C,3,B5,1,A4,2,D3,0

H,6,B6,3,A5,1,D4,0

H,6,B7,3,A6,1,D5,0

C,6,B8,3,A7,1,D6,0

O,9,B9,6,A8,3,D7,0

O,9,B10,6,A9,3,D8,0

H,9,B11,6,A10,3,D9,0

H,1,B12,10,A11,9,D10,0

H,11,B13,9,A12,6,D11,0

O,11,B14,9,A13,6,D12,0

H,15,B15,11,A14,9,D13,0

Variables:

B1 $=1.08596746$

$\mathrm{B} 2=1.53457426$

B3 $=1.08955007$

B4 $=1.09455182$

B5=1.48851792

$\mathrm{B} 6=3.85697102$

B7 $=1.07654155$

$\mathrm{B} 8=1.48857918$

B9=1.39470737

$\mathrm{B} 10=1.42707055$

B11 $=1.09191209$

$\mathrm{B} 12=1.09090211$

$\mathrm{B} 13=0.96214494$

B14 $=2.8659477$

$\mathrm{B} 15=0.96785176$

A1=114.14866589

A2 $=111.76691103$

A3 $=111.34987187$

A4 $=101.45138392$

A5 $=85.03104476$

A6 $=126.7186635$

$A 7=108.95968691$

A8 $=105.15118212$

A9 $=108.77907219$

$\mathrm{A} 10=115.27183285$

A11 $=109.99530594$

A12 $=107.87395334$

$\mathrm{A} 13=104.05101244$ 

A14=9.68134227
$\mathrm{D} 1=97.71717501$
D2 $=-23.60090562$
D3 $=-142.38980421$
D4=-82.87390989
D5 $=-165.85254167$
D6 $=7.79336256$
$\mathrm{D} 7=12.49904226$
D8 $=-106.89073227$
D9 $=130.80540226$
D10 $=-83.7159746$
D11 $=172.63019343$
D12=37.96611107
D13 $=-167.69678597$

\section{2-OH-THF••OH (TS15)}

Final structure in terms of initial Z-matrix:

C

$\mathrm{H}, 1, \mathrm{~B} 1$

$\mathrm{C}, 1, \mathrm{~B} 2,2, \mathrm{~A} 1$

H,3,B3,1,A2,2,D1,0

H,3,B4,1,A3,2,D2,0

C,3,B5,1,A4,2,D3,0

H,6,B6,3,A5,1,D4,0

H,6,B7,3,A6,1,D5,0

C,6,B8,3,A7,1,D6,0

O,9,B9,6,A8,3,D7,0

O,9,B10,6,A9,3,D8,0

H,9,B11,6,A10,3,D9,0

H,1,B12,10,A11,9,D10,0

H,11,B13,9,A12,6,D11,0

O,1,B14,10,A13,9,D12,0

H,15,B15,1,A14,10,D13,0

Variables:

B1=1.08690512

$\mathrm{B} 2=1.53120262$

B3 $=1.08603798$

B4=1.14510891

B5 $=1.53204497$

$\mathrm{B} 6=1.08591696$

B7 $=1.08802464$

$\mathrm{B} 8=1.52429407$

B9 $=1.39967654$

$\mathrm{B} 10=1.40548686$

B11=1.08995207 
B12 $=1.09208249$
B13 $=0.96106063$
B14 $=3.46672905$
B15 $=0.96970699$
A1 $=113.46166283$
A2 $=113.27466243$
A3 $=108.42968396$
A4 $=104.80834719$
A5 $=113.51158384$
A6 $=110.55178848$
A7 $=102.71789329$
A8 $=105.50558795$
A9 $=107.60325375$
A10 $=114.52227217$
A11 $=110.05238749$
A12 $=108.10545332$
A13 $=109.96301239$
A14 $=113.40486995$
D1 $=103.99675713$
D2 $=-14.64144314$
D3 $=-130.56732755$
D4 $=-129.6889256$
D5 $=107.3599682$
D6 $=-9.58593981$
D7 $=29.30042276$
D8 $=-89.71033742$
D9 $=146.43806538$
D10 $=-86.78151864$
D11 $=172.70800236$
D12 $=79.56853962$
D13 $=-139.89394064$

\section{2-OH-THF••OH (RC15)}

Final structure in terms of initial Z-matrix:

C

$\mathrm{H}, 1, \mathrm{~B} 1$

$\mathrm{C}, 1, \mathrm{~B} 2,2, \mathrm{~A} 1$

H,3,B3,1,A2,2,D1,0

H,3,B4,1,A3,2,D2,0

C,3,B5,1,A4,2,D3,0

H,6,B6,3,A5,1,D4,0

H,6,B7,3,A6,1,D5,0

C,6,B8,3,A7,1,D6,0

O,9,B9,6,A8,3,D7,0

O,9,B10,6,A9,3,D8,0 

H,9,B11,6,A10,3,D9,0
H,1,B12,10,A11,9,D10,0
H,11,B13,9,A12,6,D11,0
O,3,B14,1,A13,10,D12,0
H,15,B15,3,A14,1,D13,0
Variables:
B1 $=1.09023819$
B2 $=1.52980893$
B3 $=1.08783397$
B4 $=1.08803736$
$\mathrm{B} 5=1.53059878$
B6 $=1.08602132$
B7 $=1.08957533$
$\mathrm{B} 8=1.51886972$
B9 $=1.40047942$
$\mathrm{B} 10=1.40684447$
B11 $=1.09104985$
$\mathrm{B} 12=1.08841577$
B13 $=0.96068705$
B14 $=3.07482129$
B15 $=0.97159895$
A1 $=111.41967427$
A2 $=110.74991877$
A3 $=111.88620142$
A4 $=101.8350454$
A5 $=114.19157647$
A6 $=110.96249113$
A7 $=100.64220147$
A8 $=105.65183124$
A9 $=107.73464172$
A10 $=114.61296149$
A11 $=109.14138309$
A12 $=107.82174543$
A13 $=86.61335156$
A14 $=92.27779905$
D1 $=150.51527728$
$\mathrm{D} 2=29.24127099$
D3 $=-92.88999353$
D4 $=-156.52750333$
D5=78.5316291
D6 $=-36.5916897$
D7 $=37.49980291$
D8 $=-81.58248976$
D9 $=155.20681662$
D10 $=-123.0354429$
D11 $=167.27444125$ 
D12 $=109.11197893$

D13 $=-169.37562125$

\section{$\underline{\text { 2-OH-THF••OH (PC15) }}$}

Final structure in terms of initial Z-matrix:

C

$\mathrm{H}, 1, \mathrm{~B} 1$

C,1,B2,2,A1

H,3,B3,1,A2,2,D1,0

H,3,B4,1,A3,2,D2,0

C,3,B5,1,A4,2,D3,0

H,6,B6,3,A5,1,D4,0

H,6,B7,3,A6,1,D5,0

C,6,B8,3,A7,1,D6,0

O,9,B9,6,A8,3,D7,0

O,9,B10,6,A9,3,D8,0

H,9,B11,6,A10,3,D9,0

H,1,B12,10,A11,9,D10,0

H,11,B13,9,A12,6,D11,0

O,3,B14,1,A13,10,D12,0

H,15,B15,3,A14,1,D13,0

Variables:

B1=1.09152166

$\mathrm{B} 2=1.49046275$

B3 $=1.07698459$

B4 $=2.85835757$

B5 $=1.49231895$

B6 $=1.08934921$

B7 $=1.09321072$

$B 8=1.52949088$

B9=1.41299231

$\mathrm{B} 10=1.39864576$

$\mathrm{B} 11=1.08987252$

B12 $=1.09643939$

$\mathrm{B} 13=0.96110052$

B14 $=3.34807537$

$\mathrm{B} 15=0.96503537$

A1 $=114.48390551$

A2 $=124.10387694$

A3 $=84.02014378$

A $4=108.93089628$

A5 $=113.82413246$

A6 $=112.16256757$

A7 $=101.75594723$

A8 $=105.84064353$ 


$$
\begin{aligned}
& \text { A9 }=107.79629443 \\
& \text { A10 }=114.44372173 \\
& \text { A11 }=109.90870655 \\
& \text { A12 }=108.43543361 \\
& \text { A13 }=75.95357155 \\
& \text { A14 }=57.48278579 \\
& \text { D1 }=62.50885469 \\
& \text { D2 }=-48.0072417 \\
& \text { D3 }=-131.06431803 \\
& D 4=-125.62004039 \\
& D 5=110.92966719 \\
& D 6=-6.64709516 \\
& D 7=25.04213252 \\
& \text { D8 }=-93.71031091 \\
& \text { D9 }=141.72728504 \\
& \text { D10 }=-89.93915135 \\
& D 11=174.81045319 \\
& \text { D12 }=56.21967457 \\
& \text { D13 }=-30.55320036
\end{aligned}
$$

\section{2-OH-THF••OH (P14)}

Final structure in terms of initial Z-matrix:

C

H,1,B1

C,1,B2,2,A1

H,3,B3,1,A2,2,D1,0

C,3,B4,1,A3,2,D2,0

H,5,B5,3,A4,1,D3,0

H,5,B6,3,A5,1,D4,0

C,5,B7,3,A6,1,D5,0

O,8,B8,5,A7,3,D6,0

O,8,B9,5,A8,3,D7,0

H,8,B10,5,A9,3,D8,0

H,1,B11,9,A10,8,D9,0

H,10,B12,8,A11,5,D10,0

Variables:

B1=1.09193699

B2 $=1.48915452$

B3 $=1.07647622$

B4 $=1.4892764$

$\mathrm{B} 5=1.08921872$

B6 $=1.09479315$

$\mathrm{B} 7=1.52848979$

$\mathrm{B} 8=1.40269234$

B9 $=1.4043083$ 


$$
\begin{aligned}
& B 10=1.09032195 \\
& B 11=1.09727771 \\
& B 12=0.96091295 \\
& A 1=114.1541136 \\
& A 2=124.70819653 \\
& A 3=108.97021412 \\
& A 4=114.13773038 \\
& A 5=112.21237636 \\
& A 6=101.20988856 \\
& A 7=105.96591294 \\
& A 8=107.42584563 \\
& A 9=114.59884733 \\
& A 10=110.15108659 \\
& A 11=107.97978708 \\
& D 1=60.92549142 \\
& D 2=-125.10369283 \\
& D 3=-131.18664558 \\
& D 4=105.42992147 \\
& D 5=-11.9160129 \\
& D 6=27.8508954 \\
& D 7=-91.24124718 \\
& D 8=145.14729423 \\
& D 9=-94.29670744 \\
& D 10=171.28254632
\end{aligned}
$$

\section{2-OH-THF••OH (TS16)}

Final structure in terms of initial Z-matrix:

C

H,1,B1

$\mathrm{C}, 1, \mathrm{~B} 2,2, \mathrm{~A} 1$

H,3,B3,1,A2,2,D1,0

H,3,B4,1,A3,2,D2,0

C,3,B5,1,A4,2,D3,0

H,6,B6,3,A5,1,D4,0

H,6,B7,3,A6,1,D5,0

C,6,B8,3,A7,1,D6,0

O,9,B9,6,A8,3,D7,0

O,9,B10,6,A9,3,D8,0

H,9,B11,6,A10,3,D9,0

H,1,B12,10,A11,9,D10,0

H,11,B13,9,A12,6,D11,0

O,3,B14,1,A13,10,D12,0

H,15,B15,3,A14,1,D13,0

Variables:

B1=1.08929362 


$$
\begin{aligned}
& \text { B2 }=1.53083451 \\
& \text { B3 }=1.15351067 \\
& \text { B4 }=1.08627639 \\
& \text { B5 }=1.52482787 \\
& \text { B6 }=1.08622481 \\
& \text { B7 }=1.08991146 \\
& \text { B8 }=1.51965028 \\
& \text { B9 }=1.40086333 \\
& \text { B10 }=1.40195831 \\
& \text { B11 }=1.09082648 \\
& \text { B12 }=1.09036004 \\
& \text { B13 }=0.96080277 \\
& \text { B14 }=2.58588643 \\
& \text { B15 }=0.96943911 \\
& \text { A1 }=112.60792202 \\
& \text { A2 }=107.891601 \\
& \text { A3 }=114.23238839 \\
& \text { A4 }=104.49118194 \\
& \text { A5 }=113.67025691 \\
& \text { A6 }=110.6577633 \\
& \text { A7 }=101.41438994 \\
& \text { A8 }=105.18549587 \\
& \text { A9 }=107.49344748 \\
& \text { A10 }=114.62801335 \\
& \text { A11 }=110.18055233 \\
& \text { A12 }=108.3196353 \\
& \text { A13 }=107.1704093 \\
& \text { A14 }=93.37292183 \\
& \text { D1 }=133.41490127 \\
& \text { D2 }=14.73707029 \\
& \text { D3 }=-111.37059809 \\
& \text { D4 }=-145.55733113 \\
& \text { D5 }=90.87275606 \\
& \text { D6 }=-25.11825304 \\
& \text { D7 }=36.61642996 \\
& \text { D8 }=-82.49755691 \\
& \text { D9 }=153.49488715 \\
& \text { D10 }=-102.84302596 \\
& \text { D11 }=169.11610255 \\
& \text { D12 }=-116.52181994 \\
& \text { D13 }=-120.76891675 \\
&
\end{aligned}
$$

\section{2-OH-THF••OH (PC16)}

Final structure in terms of initial Z-matrix: C 

H,1,B1
$\mathrm{C}, 1, \mathrm{~B} 2,2, \mathrm{~A} 1$
H,3,B3,1,A2,2,D1,0
H,3,B4,1,A3,2,D2,0
C,3,B5,1,A4,2,D3,0
H,6,B6,3,A5,1,D4,0
H,6,B7,3,A6,1,D5,0
C,6,B8,3,A7,1,D6,0
O,9,B9,6,A8,3,D7,0
O,9,B10,6,A9,3,D8,0
H,9,B11,6,A10,3,D9,0
H,1,B12,10,A11,9,D10,0
H,11,B13,9,A12,6,D11,0
O,3,B14,1,A13,10,D12,0
H,15,B15,3,A14,1,D13,0
Variables:
B1 $=1.0916729$
B2 $=1.49059029$
$\mathrm{B} 3=2.90934688$
B4 $=1.07685132$
$\mathrm{B} 5=1.49008394$
B6 $=1.0897659$
B7 $=1.09470889$
$\mathrm{B} 8=1.52756738$
B9 $=1.39571549$
B10 $=1.41365152$
B11 $=1.08933704$
B12 $=1.09595359$
B13 $=0.96187681$
B14 $=3.28163858$
$\mathrm{B} 15=0.95867442$
A1 $=113.93624852$
A2 $=79.13977833$
A3 $=124.66449291$
A4 $=108.99394788$
A5 $=114.4708323$
A6 $=112.16321844$
A7 $=101.05248062$
A8 $=106.24531745$
A9 $=107.45629396$
A10 $=114.69045399$
A11 $=110.1213817$
A12 $=108.10075073$
A13 $=81.00979075$
A14 $=57.84083116$
D1 $=160.42433016$ 


$$
\begin{aligned}
& \mathrm{D} 2=50.16955193 \\
& \mathrm{D} 3=-122.19191737 \\
& \mathrm{D} 4=-133.45607735 \\
& \mathrm{D} 5=103.23399981 \\
& \mathrm{D} 6=-13.76757599 \\
& \mathrm{D} 7=28.42927827 \\
& \text { D8 }=-90.66634566 \\
& \mathrm{D} 9=146.6448309 \\
& \mathrm{D} 10=-96.27834669 \\
& \mathrm{D} 11=171.38308589 \\
& \mathrm{D} 12=-99.00839213 \\
& \mathrm{D} 13=-141.7781049
\end{aligned}
$$

\section{2-OH-THF••OH (TS17)}

Final structure in terms of initial Z-matrix:

C

$\mathrm{H}, 1, \mathrm{~B} 1$

$\mathrm{C}, 1, \mathrm{~B} 2,2, \mathrm{~A} 1$

H,3,B3,1,A2,2,D1,0

H,3,B4,1,A3,2,D2,0

C,3,B5,1,A4,2,D3,0

H,6,B6,3,A5,1,D4,0

H,6,B7,3,A6,1,D5,0

C,6,B8,3,A7,1,D6,0

O,9,B9,6,A8,3,D7,0

O,9,B10,6,A9,3,D8,0

H,9,B11,6,A10,3,D9,0

H,1,B12,10,A11,9,D10,0

H,11,B13,9,A12,6,D11,0

O,1,B14,10,A13,9,D12,0

H,15,B15,1,A14,10,D13,0

Variables:

B1=1.14459637

$\mathrm{B} 2=1.51974782$

B3 $=1.08908762$

B4 $=1.08631429$

B5=1.52974431

B6 $=1.08544359$

B7 $=1.0889357$

$\mathrm{B} 8=1.51777639$

B9 $=1.41545778$

$\mathrm{B} 10=1.39982148$

$\mathrm{B} 11=1.09070728$

$\mathrm{B} 12=1.08853959$

$\mathrm{B} 13=0.9607707$ 


$$
\begin{aligned}
& B 14=2.5814138 \\
& B 15=0.97053448 \\
& A 1=108.35719529 \\
& A 2=110.41791058 \\
& A 3=112.06599965 \\
& A 4=101.06320748 \\
& A 5=114.22367304 \\
& A 6=110.24685474 \\
& A 7=101.08637269 \\
& A 8=105.49871315 \\
& A 9=108.20269881 \\
& A 10=114.82202963 \\
& A 11=110.24062433 \\
& A 12=108.44099633 \\
& A 13=93.11601523 \\
& A 14=82.59473607 \\
& D 1=154.84530923 \\
& D 2=32.82702645 \\
& D 3=-88.24228728 \\
& D 4=-156.99057469 \\
& D 5=78.64144158 \\
& \text { D6 }=-37.13882952 \\
& D 7=35.34053899 \\
& D 8=-83.00203602 \\
& D 9=152.7872948 \\
& D 10=-133.26323064 \\
& D 11=170.14500484 \\
& D 12=102.06399049 \\
& D 13=15.19520472
\end{aligned}
$$

\section{2-OH-THF••OH (PC17)}

Final structure in terms of initial Z-matrix:

C

$\mathrm{H}, 1, \mathrm{~B} 1$

$\mathrm{C}, 1, \mathrm{~B} 2,2, \mathrm{~A} 1$

H,3,B3,1,A2,2,D1,0

$\mathrm{H}, 3, \mathrm{~B} 4,1, \mathrm{~A} 3,4, \mathrm{D} 2,0$

C,3,B5,1,A4,4,D3,0

H,6,B6,3,A5,1,D4,0

H,6,B7,3,A6,1,D5,0

C,6,B8,3,A7,1,D6,0

O,1,B9,3,A8,6,D7,0

O,9,B10,6,A9,3,D8,0

H,9,B11,6,A10,3,D9,0

H,1,B12,10,A11,9,D10,0 
H,11,B13,9,A12,6,D11,0 O,10,B14,1,A13,3,D12,0

H,15,B15,10,A14,1,D13,0

Variables:

B1 $=3.87010255$

B2 $=1.49121003$

B3 $=1.09469216$

B4 $=1.08804608$

B5 $=1.53509025$

B6 $=1.08525206$

B7 $=1.08766939$

B8 $=1.51813923$

$\mathrm{B} 9=1.37437452$

B10 $=1.39377306$

B11 $=1.08924152$

B12 $=1.07859966$

$\mathrm{B} 13=0.96071208$

$\mathrm{B} 14=2.86824321$

B15 $=0.96408098$

A1 $=106.27044558$

A2 $=111.67136414$

A3 $=112.67412844$

A4 $=100.94981698$

A5 $=114.04251178$

A6 $=110.58975266$

A7 $=101.88257762$

A8 $=110.77650097$

A9 $=108.71462346$

A10 $=114.78935722$

A11 $=114.96304729$

A12 $=108.86671101$

A13 $=100.96668504$

A14 $=24.26355532$

D1 $=-165.4613445$

D2 $=-121.76753521$

D3 $=117.43718585$

D4 $=-152.24835362$

D5 $=82.91042599$

D6 $=-32.56732037$

D7 $=21.8658643$

D8 $=-83.92421746$

D9=149.90764099

D10 $=-153.26324952$

D11 $=174.62307463$

D12 $=-89.39175568$

D13=-78.82348659 


\section{2-OH-THF••OH (P15)}

Final structure in terms of initial Z-matrix:

C

$\mathrm{H}, 1, \mathrm{~B} 1$

$\mathrm{C}, 1, \mathrm{~B} 2,2, \mathrm{~A} 1$

$\mathrm{H}, 3, \mathrm{~B} 3,1, \mathrm{~A} 2,2, \mathrm{D} 1,0$

H,3,B4,1,A3,2,D2,0

C,3,B5,1,A4,2,D3,0

H,6,B6,3,A5,1,D4,0

H,6,B7,3,A6,1,D5,0

C,6,B8,3,A7,1,D6,0

O,1,B9,3,A8,6,D7,0

O,9,B10,6,A9,3,D8,0

H,9,B11,6,A10,3,D9,0

H,11,B12,9,A11,6,D10,0

Variables:

B1=1.0775092

B2 $=1.49720377$

B3 $=1.09235954$

B4 $=1.0889045$

B5 $=1.53846952$

B6=1.08548671

$\mathrm{B} 7=1.08833348$

$\mathrm{B} 8=1.51965248$

B9=1.37092857

$\mathrm{B} 10=1.39766639$

$\mathrm{B} 11=1.09013414$

$\mathrm{B} 12=0.96093936$

A1 $=127.00499028$

A2 $=111.37621946$

A3 $=112.78369415$

A4 $=101.89474857$

A5 $=114.08777$

A6 $=110.6415107$

A7 $=101.87781085$

A8 $=111.01880631$

A9 $=108.26961076$

$\mathrm{A} 10=115.06549334$

A11 $=108.47211289$

D1=103.98107512

D2 $=-17.28377249$

D3 $=-138.35911902$

D4=-146.19029513

D5 $=89.80610904$ 


$$
\begin{aligned}
& \mathrm{D} 6=-26.14906418 \\
& \mathrm{D} 7=9.6193505 \\
& \mathrm{D} 8=-83.64082532 \\
& \mathrm{D} 9=151.20553349 \\
& \mathrm{D} 10=168.36428645
\end{aligned}
$$

\section{$\underline{\text { 2-OH-THF••OH (TS18) }}$}

Final structure in terms of initial Z-matrix:

C

H,1,B1

C,1,B2,2,A1

H,3,B3,1,A2,2,D1,0

H,3,B4,1,A3,2,D2,0

C,3,B5,1,A4,2,D3,0

H,6,B6,3,A5,1,D4,0

H,6,B7,3,A6,1,D5,0

C,6,B8,3,A7,1,D6,0

O,1,B9,3,A8,6,D7,0

O,9,B10,6,A9,3,D8,0

H,9,B11,6,A10,3,D9,0

H,1,B12,10,A11,9,D10,0

H,11,B13,9,A12,6,D11,0

O,1,B14,10,A13,9,D12,0

H,15,B15,1,A14,10,D13,0

Variables:

B1=1.08683392

B2 $=1.51656916$

B3 $=1.08619642$

B4 $=1.08970565$

B5 $=1.5366098$

B6 $=1.0874549$

$\mathrm{B} 7=1.08606349$

$\mathrm{B} 8=1.53554873$

B9 $=1.40325323$

$\mathrm{B} 10=1.41022245$

$\mathrm{B} 11=1.08948973$

B12 $=1.15951253$

$\mathrm{B} 13=0.96159451$

B14 $=2.57657728$

$\mathrm{B} 15=0.9738571$

A1 $=117.27499857$

A2 $=112.23780053$

A3 $=109.79522759$

A4=101.63405892

A5 $=111.36691071$ 


$$
\begin{aligned}
& A 6=112.77632667 \\
& A 7=104.08731261 \\
& A 8=105.28304453 \\
& A 9=108.31331023 \\
& A 10=114.50998247 \\
& A 11=109.81030481 \\
& A 12=108.23348351 \\
& A 13=104.88855041 \\
& A 14=86.33694155 \\
& D 1=82.28230599 \\
& D 2=-39.228156 \\
& D 3=-156.94685097 \\
& D 4=-97.41414913 \\
& D 5=140.27875282 \\
& \text { D6 }=19.29171223 \\
& D 7=-35.10455114 \\
& D 8=-117.01278111 \\
& D 9=120.08112486 \\
& D 10=-77.33420357 \\
& D 11=178.06549699 \\
& D 12=-64.78665282 \\
& D 13=21.72981555
\end{aligned}
$$

\section{$\underline{\text { 2-OH-THF••OH (PC18) }}$}

Final structure in terms of initial Z-matrix:

C

$\mathrm{H}, 1, \mathrm{~B} 1$

C,1,B2,2,A1

H,3,B3,1,A2,2,D1,0

H,3,B4,1,A3,2,D2,0

C,3,B5,1,A4,2,D3,0

H,6,B6,3,A5,1,D4,0

H,6,B7,3,A6,1,D5,0

C,6,B8,3,A7,1,D6,0

O,1,B9,3,A8,6,D7,0

O,9,B10,6,A9,3,D8,0

H,9,B11,6,A10,3,D9,0

H,1,B12,10,A11,9,D10,0

H,11,B13,9,A12,6,D11,0

O,1,B14,10,A13,9,D12,0

H,15,B15,1,A14,10,D13,0

Variables:

B1 $=1.07838376$

$\mathrm{B} 2=1.49920385$

B3 $=1.09188243$ 


$$
\begin{aligned}
& \text { B4=1.08939014 } \\
& \text { B5 }=1.54013074 \\
& \text { B6 }=1.08551374 \\
& \text { B7 }=1.08792197 \\
& \text { B8 }=1.51981268 \\
& \text { B9 }=1.37075911 \\
& \text { B10 }=1.40423446 \\
& \text { B11 }=1.08887727 \\
& \text { B12 }=2.67166777 \\
& \text { B13 }=0.96193396 \\
& \text { B14 }=3.26994201 \\
& \text { B15 }=0.96435653 \\
& \text { A1 }=126.57495841 \\
& \text { A2 }=111.36081595 \\
& \text { A3 }=112.47147491 \\
& \text { A4 }=102.1161593 \\
& \text { A5 }=113.99074093 \\
& \text { A6 }=110.65128019 \\
& \mathrm{~A} 7=102.66801191 \\
& \text { A8 }=111.13880486 \\
& \text { A9 }=108.6958551 \\
& \text { A10 }=114.99769872 \\
& \text { A11 }=104.32964037 \\
& \text { A12 }=108.66458608 \\
& \text { A13 }=98.31923674 \\
& \text { A14 }=63.45030734 \\
& \text { D1 }=99.93042976 \\
& \text { D2 }=-20.94741744 \\
& \text { D3 }=-141.44649386 \\
& \text { D4 }=-142.40923436 \\
& \text { D5 }=94.0035543 \\
& \text { D6 }=-22.06852213 \\
& \text { D7 }=5.20131038 \\
& \text { D8 }=-86.35043051 \\
& \text { D9 }=149.13497604 \\
& \text { D10 }=-80.69061189 \\
& \text { D11 }=175.46859406 \\
& \text { D12 }=-67.09908786 \\
& \text { D13 }=35.74072262
\end{aligned}
$$

\section{$\underline{\mathrm{HO}_{2}}$}

Final structure in terms of initial Z-matrix:

$\mathrm{O}$

$$
\mathrm{H}, 1, \mathrm{~B} 1
$$

$\mathrm{O}, 1, \mathrm{~B} 2,2, \mathrm{~A} 1$

Variables: 
$\mathrm{B} 1=0.97198957$

$\mathrm{B} 2=1.30369402$

A1 $=105.95837081$

\section{$\underline{2-\mathrm{OH}-\mathrm{THF} \cdot \bullet \mathrm{O}_{2}\left(\mathrm{RO}_{2}\right)}$}

Final structure in terms of initial Z-matrix:

C

$\mathrm{H}, 1, \mathrm{~B} 1$

$\mathrm{C}, 1, \mathrm{~B} 2,2, \mathrm{~A} 1$

H,3,B3,1,A2,2,D1,0

H,3,B4,1,A3,2,D2,0

C,3,B5,1,A4,2,D3,0

H,6,B6,3,A5,1,D4,0

H,6,B7,3,A6,1,D5,0

C,6,B8,3,A7,1,D6,0

O,1,B9,3,A8,6,D7,0

O,9,B10,6,A9,3,D8,0

H,9,B11,6,A10,3,D9,0

H,11,B12,9,A11,6,D10,0

O,1,B13,10,A12,9,D11,0

O,14,B14,1,A13,10,D12,0

Variables:

B1 $=1.08716068$

$\mathrm{B} 2=1.5326565$

B3 $=1.08674427$

B4 $=1.08524257$

B5 $=1.5351766$

B6 $=1.08545693$

B7 $=1.0878093$

B8 $=1.51766638$

B9=1.38107016

$\mathrm{B} 10=1.39372811$

$\mathrm{B} 11=1.08967489$

$\mathrm{B} 12=0.96136277$

$\mathrm{B} 13=1.45966373$

B14=1.29128675

A1 $=115.67734624$

A2 $=108.87592405$

A3 $=111.50182153$

A4 $=103.07273853$

A5 $=113.42286049$

A6 $=111.18120071$

A7 $=101.84301576$

A8 $=108.08372763$

A9 $=108.10423853$ 


$$
\begin{aligned}
& A 10=115.28737367 \\
& A 11=108.97394168 \\
& A 12=106.78022801 \\
& A 13=110.24992861 \\
& D 1=121.5045621 \\
& D 2=1.89756123 \\
& D 3=-119.72091379 \\
& D 4=-144.03990742 \\
& D 5=92.21103789 \\
& D 6=-24.1594651 \\
& D 7=4.25159162 \\
& D 8=-82.20413166 \\
& D 9=151.91134346 \\
& D 10=166.03468968 \\
& D 11=-99.43971272 \\
& D 12=-172.50233648
\end{aligned}
$$

\section{$\underline{\text { 2-OH-THF } \bullet \bullet \mathrm{O}_{2}}$ (TS19)}

Final structure in terms of initial Z-matrix:

C

$\mathrm{H}, 1, \mathrm{~B} 1$

C,1,B2,2,A1

H,3,B3,1,A2,2,D1,0

H,3,B4,1,A3,2,D2,0

C,3,B5,1,A4,2,D3,0

H,6,B6,3,A5,1,D4,0

H,6,B7,3,A6,1,D5,0

C,6,B8,3,A7,1,D6,0

O,1,B9,3,A8,6,D7,0

O,9,B10,6,A9,3,D8,0

H,9,B11,6,A10,3,D9,0

H,11,B12,9,A11,6,D10,0

O,1,B13,10,A12,9,D11,0

O,14,B14,1,A13,10,D12,0

Variables:

B1 $=1.08821274$

B2 $=1.50882773$

$\mathrm{B} 3=1.0859541$

B4=1.09006227

B5 $=1.53122981$

B6 $=1.08701435$

B7 $=1.08765909$

B8 $=1.52237355$

B9=1.24046199

$\mathrm{B} 10=1.2630554$ 


$$
\begin{aligned}
& \text { B11 }=1.09162504 \\
& \text { B12 }=1.26003639 \\
& \text { B13 }=2.06575066 \\
& \text { B14 }=1.28924819 \\
& \text { A1 }=121.32482675 \\
& \text { A2 }=111.13008912 \\
& \text { A3 }=108.4483979 \\
& \text { A4 }=103.75577969 \\
& \text { A5 }=112.35914435 \\
& \text { A6 }=110.49776802 \\
& \text { A7 }=107.56744988 \\
& \text { A8 }=115.86814698 \\
& \text { A9 }=120.20467339 \\
& \text { A10 }=116.27497284 \\
& \text { A11 }=116.23091298 \\
& \text { A12 }=107.77363985 \\
& \text { A13 }=115.82431621 \\
& \text { D1 }=74.55908296 \\
& \text { D2 }=-43.25880711 \\
& \text { D3 }=-162.43522273 \\
& \text { D4 }=-143.52568582 \\
& \text { D5 }=94.61650328 \\
& \text { D6 }=-24.42746093 \\
& \text { D7 }=-3.42074995 \\
& \text { D8 }=-76.80227983 \\
& \text { D9 }=133.22840452 \\
& \text { D10 }=54.4112417 \\
& \text { D11 }=-81.6217927 \\
& \text { D12 }=55.48098166
\end{aligned}
$$

\section{$\underline{\text { 2-OH-THF } \bullet \bullet \mathrm{O}_{2}} \underline{\text { (PC19) }}$}

Final structure in terms of initial Z-matrix:

$\mathrm{C}$

$\mathrm{H}, 1, \mathrm{~B} 1$

$\mathrm{C}, 1, \mathrm{~B} 2,2, \mathrm{~A} 1$

H,3,B3,1,A2,2,D1,0

H,3,B4,1,A3,2,D2,0

C,3,B5,1,A4,2,D3,0

H,6,B6,3,A5,1,D4,0

H,6,B7,3,A6,1,D5,0

$\mathrm{C}, 6, \mathrm{~B} 8,3, \mathrm{~A} 7,1, \mathrm{D} 6,0$

$\mathrm{O}, 1, \mathrm{~B} 9,3, \mathrm{~A} 8,6, \mathrm{D} 7,0$

O,9,B10,6,A9,3,D8,0

H,9,B11,6,A10,3,D9,0

H,11,B12,9,A11,6,D10,0 


$$
\begin{aligned}
& \text { O,1,B13,10,A12,3,D11,0 } \\
& \text { O,14,B14,1,A13,10,D12,0 } \\
& \text { Variables: } \\
& \text { B1 }=1.1005416 \\
& \text { B2 }=1.51009832 \\
& \text { B3 }=1.09096007 \\
& \text { B4 }=1.09195789 \\
& \text { B5 }=1.51516769 \\
& \text { B6 }=1.09437597 \\
& \text { B7 }=1.0907726 \\
& \text { B8 }=1.49898748 \\
& \text { B9 }=1.19949014 \\
& \text { B10 }=1.20361193 \\
& \text { B11 }=1.10204901 \\
& \text { B12 }=1.73063162 \\
& \text { B13 }=2.87698563 \\
& \text { B14 }=1.30192998 \\
& \text { A1 }=115.95468847 \\
& \text { A2 }=107.82439861 \\
& \text { A3 }=107.88268214 \\
& \text { A4 }=111.28768803 \\
& \text { A5 }=111.45404224 \\
& \text { A6 }=111.30562085 \\
& \text { A7 }=113.69546151 \\
& \text { A8 }=122.75026588 \\
& \text { A9 }=124.98583116 \\
& \text { A10 }=115.7864891 \\
& \text { A11 }=145.15429399 \\
& \text { A12 }=114.47034508 \\
& \text { A13 }=113.2246292 \\
& \text { D1 }=57.89061086 \\
& \text { D2 }=-56.58647749 \\
& \text { D3 }=-178.63500147 \\
& \text { D4 }=174.159979 \\
& \text { D5 }=55.55115381 \\
& \text { D6 }=-65.87773326 \\
& \text { D7 }=0.18345809 \\
& \text { D8 }=-16.72457016 \\
& \text { D9 }=165.44316226 \\
& \text { D10 }=9.052213 \\
& \text { D11 }=-103.96781094 \\
& \text { D12 }=56.66713955 \\
&
\end{aligned}
$$

\section{Succinaldehyde}

Final structure in terms of initial Z-matrix: 
C

H,1,B1

C,1,B2,2,A1

H,3,B3, 1,A2,2,D1,0

H,3,B4,1,A3,2,D2,0

C,3,B5,1,A4,2,D3,0

H,6,B6,3,A5,1,D4,0

H,6,B7,3,A6,1,D5,0

C,6,B8,3,A7,1,D6,0

O,1,B9,3,A8,6,D7,0

O,9,B10,6,A9,3,D8,0

H,9,B11,6,A10,3,D9,0

Variables:

B1=1.10494917

B2 $=1.50718057$

B3 $=1.09120957$

B4 $=1.09292198$

B5 $=1.51449123$

$\mathrm{B} 6=1.09287713$

B7 $=1.09130071$

$\mathrm{B} 8=1.50736155$

B9 $=1.1977407$

$\mathrm{B} 10=1.1977876$

$\mathrm{B} 11=1.10496374$

A1=115.59610781

A2 $=107.78330465$

$\mathrm{A} 3=107.31888804$

A4 $=111.8010373$

A5 $=111.72066465$

$\mathrm{A} 6=111.24741357$

A7 $=111.79840038$

A8 $=123.76341209$

A9 $=123.76448415$

A10=115.60766956

$\mathrm{D} 1=49.36692866$

D2 $=-65.23434545$

D3 $=171.9883762$

D4 $=170.39977977$

D5 $=51.24127086$

D6 $=-69.27154444$

D7 $=-9.12511623$

D8 $=-8.39669492$

D9=172.66790624

$\underline{2-\mathrm{OH}-\mathrm{THF} \cdot \bullet \bullet \mathrm{O}_{2}}$ (TS20) 
Final structure in terms of initial Z-matrix:

C

$\mathrm{H}, 1, \mathrm{~B} 1$

$\mathrm{C}, 1, \mathrm{~B} 2,2, \mathrm{~A} 1$

$\mathrm{H}, 3, \mathrm{~B} 3,1, \mathrm{~A} 2,2, \mathrm{D} 1,0$

H,3,B4,1,A3,2,D2,0

C,3,B5,1,A4,2,D3,0

H,6,B6,3,A5,1,D4,0

H,6,B7,3,A6,1,D5,0

C,6,B8,3,A7,1,D6,0

O,1,B9,3,A8,6,D7,0

O,9,B10,6,A9,3,D8,0

H,9,B11,6,A10,3,D9,0

H,11,B12,9,A11,6,D10,0

O,1,B13,10,A12,9,D11,0

O,14,B14,1,A13,10,D12,0

Variables:

$\mathrm{B} 1=1.07705223$

B2 $=1.39145092$

$\mathrm{B} 3=1.36688246$

B4=1.08042685

$\mathrm{B} 5=1.52493255$

$\mathrm{B} 6=1.08657579$

$\mathrm{B} 7=1.08957107$

$B 8=1.52454523$

B9=1.33794302

$\mathrm{B} 10=1.38699521$

B11 $=1.08910475$

$\mathrm{B} 12=0.96154053$

$\mathrm{B} 13=2.13249683$

B14=1.26138188

A1 $=129.03422322$

A2 $=93.79857931$

A3 $=119.82988249$

A4=105.20537582

A5 $=114.64305935$

A6 $=111.4379466$

A7 $=100.96528114$

A8 $=113.79610918$

A9=108.25452889

$\mathrm{A} 10=115.77135343$

A11=109.35507819

A12 $=100.43378636$

A13 $=100.68191633$

D1=93.93644498

D2=-14.19213431 


$$
\begin{aligned}
& D 3=-155.66784248 \\
& D 4=-138.38514604 \\
& D 5=96.99910485 \\
& D 6=-20.76529311 \\
& D 7=7.22594962 \\
& D 8=-90.27669837 \\
& D 9=142.95232418 \\
& D 10=165.56386269 \\
& D 11=-91.36889742 \\
& D 12=113.92532201
\end{aligned}
$$

\section{$\underline{2-\mathrm{OH}-\mathrm{THF} \cdot \bullet \mathrm{O}_{2}}$ (PC20)}

Final structure in terms of initial Z-matrix:

C

$\mathrm{H}, 1, \mathrm{~B} 1$

$\mathrm{C}, 1, \mathrm{~B} 2,2, \mathrm{~A} 1$

H,3,B3,1,A2,2,D1,0

H,3,B4,1,A3,2,D2,0

C,3,B5,1,A4,2,D3,0

H,6,B6,3,A5,1,D4,0

H,6,B7,3,A6,1,D5,0

C,6,B8,3,A7,1,D6,0

O,1,B9,3,A8,6,D7,0

O,9,B10,6,A9,3,D8,0

H,9,B11,6,A10,3,D9,0

H,11,B12,9,A11,6,D10,0

O,1,B13,3,A12,6,D11,0

O,14,B14,1,A13,3,D12,0

Variables:

B1=1.07764639

B2 $=1.32415652$

B3 $=3.00723981$

B4 $=1.07560798$

$\mathrm{B} 5=1.50856676$

$\mathrm{B} 6=1.08790055$

$\mathrm{B} 7=1.09061518$

$\mathrm{B} 8=1.52935389$

B9=1.37057659

$\mathrm{B} 10=1.40284419$

$\mathrm{B} 11=1.08787744$

$\mathrm{B} 12=0.96729487$

B13=3.84652626

B14=1.3012195

A1=130.69137809

$\mathrm{A} 2=64.40176936$ 


$$
\begin{aligned}
& A 3=125.86013204 \\
& A 4=108.06645362 \\
& A 5=114.39660367 \\
& A 6=111.67015437 \\
& A 7=100.73207121 \\
& A 8=114.18719643 \\
& A 9=107.75886217 \\
& A 10=115.66270253 \\
& A 11=109.24518401 \\
& A 12=114.43870187 \\
& A 13=52.073379 \\
& D 1=109.03427277 \\
& D 2=-0.66848895 \\
& D 3=-178.02517077 \\
& D 4=-130.82645373 \\
& D 5=105.44125655 \\
& D 6=-13.30954414 \\
& D 7=1.55967689 \\
& D 8=-96.62426788 \\
& D 9=138.73367799 \\
& D 10=169.70197112 \\
& D 11=67.89539369 \\
& D 12=53.04036663
\end{aligned}
$$

\section{2,3-dihydro-2-furanol}

Final structure in terms of initial Z-matrix:

C

H,1,B1

$\mathrm{C}, 1, \mathrm{~B} 2,2, \mathrm{~A} 1$

H,3,B3,1,A2,2,D1,0

C,3,B4,1,A3,2,D2,0

$\mathrm{H}, 5, \mathrm{~B} 5,3, \mathrm{~A} 4,1, \mathrm{D} 3,0$

$\mathrm{H}, 5, \mathrm{~B} 6,3, \mathrm{~A} 5,1, \mathrm{D} 4,0$

C,5,B7,3,A6,1,D5,0

$\mathrm{O}, 1, \mathrm{~B} 8,3, \mathrm{~A} 7,5, \mathrm{D} 6,0$

O,8,B9,5,A8,3,D7,0

H,8,B10,5,A9,3,D8,0

H,10,B11,8,A10,5,D9,0

Variables:

B1 $=1.07767463$

B2 $=1.32270423$

B3 $=1.07567939$

B4=1.50683794

$\mathrm{B} 5=1.08827626$

B6 $=1.09049342$ 

B7 $=1.53184109$
B8 $=1.36863963$
B9 $=1.39381421$
B10 $=1.08976251$
B11 $=0.96104304$
A1 $=130.41010469$
$\mathrm{A} 2=125.88281823$
A $3=108.20724048$
A4 $=113.92074584$
A5 $=111.90394046$
A6 $=101.31555956$
$\mathrm{A} 7=114.49922707$
A8 $=108.31454304$
A9 $=114.73909121$
A10 $=108.51922004$
$\mathrm{D} 1=0.27238505$
D2 $=-178.58419797$
D3 $=-127.4703988$
D4 $=108.97426934$
D5 $=-10.25565136$
D6 $=0.459992$
D7 $=-101.88148439$
D8 $=133.38099688$
D9 $=171.04932052$ 
Table S6. The L-J parameters used in the various species catalyzed by the cyclization of 4$\mathrm{OH}-\mathrm{BL}$ and the 2-OH-THF radical $+{ }^{3} \mathrm{O}_{2}$ reaction systems.

\begin{tabular}{|l|c|c|c|}
\hline Reaction system & $\sigma(\AA)$ & $\varepsilon(\mathrm{K})$ & Corresponding Alkane \\
\hline 4-OH-BL $+\mathrm{H}_{2} \mathrm{O}$ & 4.5 & 387.6 & Nonane \\
\hline 4-OH-BL $+\mathrm{HO}_{2}$ & 4.6 & 400.8 & Decane \\
\hline 4-OH-BL + Formic acid & 4.7 & 437.7 & Dodecane \\
\hline 4-OH-BL $+\mathrm{HNO}_{3}$ & 4.7 & 437.7 & Dodecane \\
\hline 4-OH-BL $+\mathrm{H}_{2} \mathrm{SO}_{4}$ & 4.7 & 437.7 & Dodecane \\
\hline 2-OH-THF radical $+{ }^{3} \mathrm{O}_{2}$ & 4.4 & 358.5 & Heptane \\
\hline
\end{tabular}

Table S7. Bimolecular rate constants $\left(k\right.$ in $\mathrm{cm}^{3}$ molecule $\left.^{-1} \mathrm{~s}^{-1}\right)$ for the cyclization of 4hydroxybutanal in presence of various catalysts and effective first order rate constant $\left(k_{\mathrm{cis}-\mathrm{H}_{2} \mathrm{SO}_{4}}^{\mathrm{eff}}\right.$ in $\mathrm{s}^{-1}$ ) for the cyclization of 4-hydroxybutanal catalyzed by $c i s-\mathrm{H}_{2} \mathrm{SO}_{4}$ over the temperatures between 200 and $300 \mathrm{~K}$.

\begin{tabular}{|c|c|c|c|c|c|c|c|}
\hline $\mathrm{T}(\mathrm{K})$ & $k_{\mathrm{H}_{2} \mathrm{O}}$ & $k_{\mathrm{HO}_{2}}$ & $k_{\mathrm{FA}}$ & $k_{\mathrm{HNO}_{3}}$ & $k_{\text {trans }-\mathrm{H}_{2} \mathrm{SO}_{4}}$ & $k_{\text {cis- } \mathrm{H}_{2} \mathrm{SO}_{4}}$ & $k_{\text {cis- }}$ eff \\
\hline 200 & $1.7 \times 10^{-27}$ & $3.9 \times 10^{-12}$ & $9.8 \times 10^{-13}$ & $8.2 \times 10^{-12}$ & $9.5 \times 10^{-11}$ & $9.8 \times 10^{-11}$ & $9.8 \times 10^{-5}$ \\
\hline 220 & $4.7 \times 10^{-27}$ & $3.4 \times 10^{-12}$ & $9.5 \times 10^{-13}$ & $7.1 \times 10^{-12}$ & $9.0 \times 10^{-11}$ & $9.4 \times 10^{-11}$ & $9.4 \times 10^{-5}$ \\
\hline 240 & $1.5 \times 10^{-26}$ & $2.8 \times 10^{-12}$ & $9.1 \times 10^{-13}$ & $5.9 \times 10^{-12}$ & $8.4 \times 10^{-11}$ & $9.0 \times 10^{-11}$ & $9.0 \times 10^{-5}$ \\
\hline 250 & $2.8 \times 10^{-26}$ & $2.5 \times 10^{-12}$ & $8.8 \times 10^{-13}$ & $5.3 \times 10^{-12}$ & $8.0 \times 10^{-11}$ & $8.7 \times 10^{-11}$ & $8.7 \times 10^{-5}$ \\
\hline 260 & $5.3 \times 10^{-26}$ & $2.2 \times 10^{-12}$ & $8.5 \times 10^{-13}$ & $4.7 \times 10^{-12}$ & $7.6 \times 10^{-11}$ & $8.4 \times 10^{-11}$ & $8.4 \times 10^{-5}$ \\
\hline 280 & $1.8 \times 10^{-25}$ & $1.7 \times 10^{-12}$ & $7.7 \times 10^{-13}$ & $3.6 \times 10^{-12}$ & $6.9 \times 10^{-11}$ & $7.6 \times 10^{-11}$ & $7.6 \times 10^{-5}$ \\
\hline 300 & $5.9 \times 10^{-25}$ & $1.3 \times 10^{-12}$ & $6.8 \times 10^{-13}$ & $2.6 \times 10^{-12}$ & $5.8 \times 10^{-11}$ & $6.8 \times 10^{-11}$ & $6.8 \times 10^{-5}$ \\
\hline
\end{tabular}


Table S8. Bimolecular rate constants $\left(k\right.$ in $\mathrm{cm}^{3}$ molecule $\left.^{-1} \mathrm{~s}^{-1}\right)$ without tunneling contribution for the cyclization of 4-hydroxybutanal in presence of various catalysts over the temperatures between 200 and $300 \mathrm{~K}$.

\begin{tabular}{|c|c|c|c|c|c|}
\hline $\mathrm{T}(\mathrm{K})$ & $k_{\mathrm{H}_{2} \mathrm{O}}$ & $k_{\mathrm{HO}_{2}}$ & $k_{\mathrm{FA}}$ & $k_{\mathrm{HNO}_{3}}$ & $k_{\text {trans- } \mathrm{H}_{2} \mathrm{SO}_{4}}$ \\
\hline 200 & $1.7 \times 10^{-30}$ & $3.4 \times 10^{-14}$ & $3.2 \times 10^{-13}$ & $3.7 \times 10^{-12}$ & $9.5 \times 10^{-11}$ \\
\hline 220 & $3.5 \times 10^{-29}$ & $4.0 \times 10^{-14}$ & $3.9 \times 10^{-13}$ & $3.6 \times 10^{-12}$ & $9.0 \times 10^{-11}$ \\
\hline 240 & $4.3 \times 10^{-28}$ & $4.0 \times 10^{-14}$ & $4.3 \times 10^{-13}$ & $3.2 \times 10^{-12}$ & $8.3 \times 10^{-11}$ \\
\hline 250 & $1.3 \times 10^{-27}$ & $3.9 \times 10^{-14}$ & $4.4 \times 10^{-13}$ & $2.9 \times 10^{-12}$ & $8.0 \times 10^{-11}$ \\
\hline 260 & $3.6 \times 10^{-27}$ & $3.6 \times 10^{-14}$ & $4.4 \times 10^{-13}$ & $2.7 \times 10^{-12}$ & $7.6 \times 10^{-11}$ \\
\hline 280 & $2.2 \times 10^{-26}$ & $3.3 \times 10^{-14}$ & $4.5 \times 10^{-13}$ & $2.1 \times 10^{-12}$ & $6.7 \times 10^{-11}$ \\
\hline 300 & $1.0 \times 10^{-25}$ & $2.8 \times 10^{-14}$ & $4.7 \times 10^{-13}$ & $1.6 \times 10^{-12}$ & $5.8 \times 10^{-11}$ \\
\hline
\end{tabular}

Table S9. Bimolecular rate constants $\left(k_{\mathrm{HNO}_{3}}\right.$ in $\mathrm{cm}^{3}$ molecule $\left.\mathrm{e}^{-1} \mathrm{~s}^{-1}\right)$ and effective first order rate constants $\left(k_{\mathrm{HNO}_{3}}^{\text {eff }}\right.$ in s$\left.{ }^{-1}\right)$ for the dehydration of 2-hydroxytetrahydrofuran in presence of nitric acid catalyst over the temperatures between 200 and $300 \mathrm{~K}$.

\begin{tabular}{|c|c|c|}
\hline $\mathrm{T}(\mathrm{K})$ & $k_{\mathrm{HNO}_{3}}$ & $k_{\mathrm{HNO}_{3}}^{\text {eff }}$ \\
\hline 200 & $1.1 \times 10^{-38}$ & $4.4 \times 10^{-28}$ \\
\hline 220 & $1.8 \times 10^{-36}$ & $7.3 \times 10^{-26}$ \\
\hline 240 & $1.3 \times 10^{-34}$ & $5.4 \times 10^{-24}$ \\
\hline 250 & $9.0 \times 10^{-34}$ & $3.6 \times 10^{-23}$ \\
\hline 260 & $5.3 \times 10^{-33}$ & $2.1 \times 10^{-22}$ \\
\hline 280 & $1.3 \times 10^{-31}$ & $5.0 \times 10^{-21}$ \\
\hline 300 & $2.0 \times 10^{-30}$ & $8.1 \times 10^{-20}$ \\
\hline
\end{tabular}




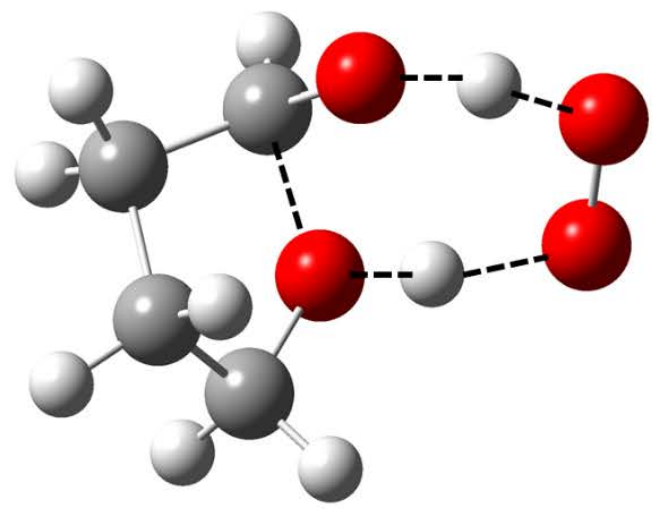

TS3a

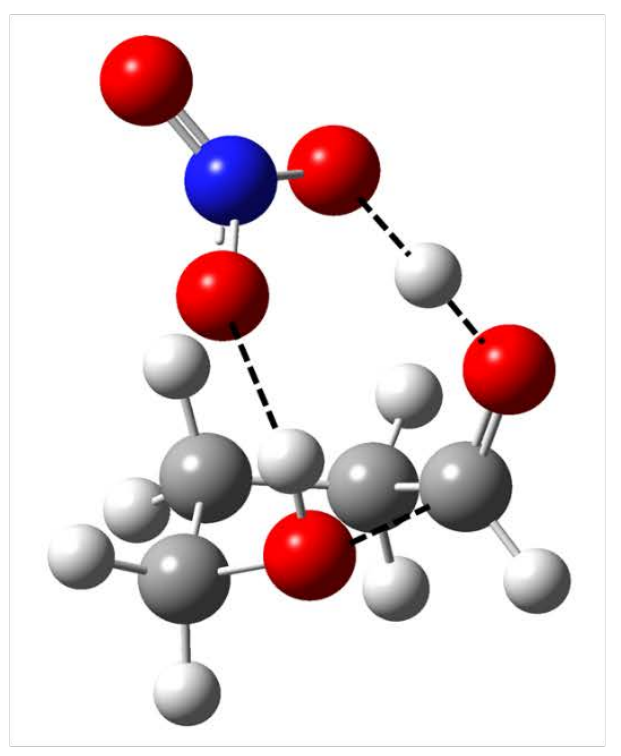

TS7a

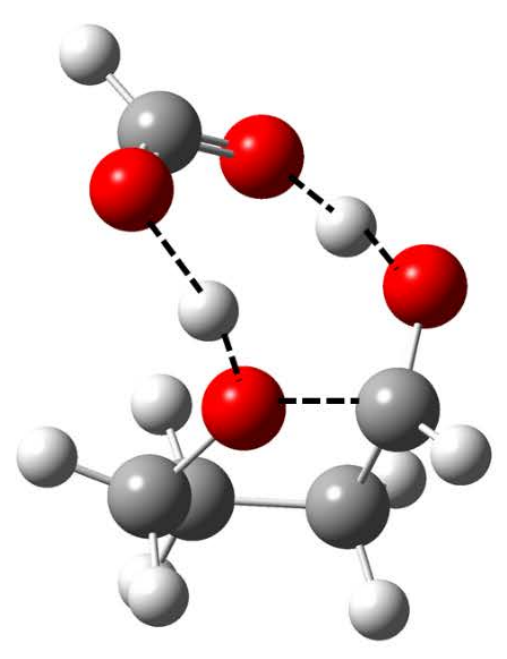

TS5a

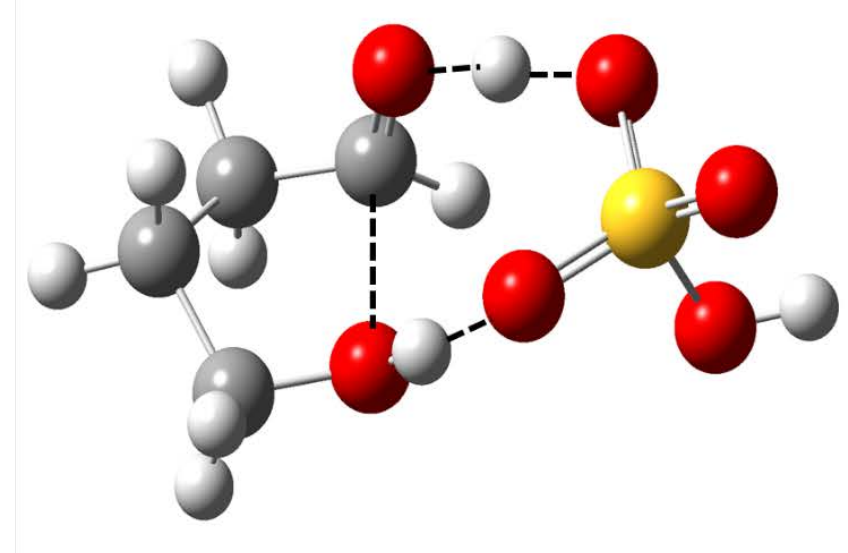

TS9a

Figure S1. Optimized geometries of the high energy transition states for the cyclization of 4hydroxybutanal assisted by hydroperoxyl $\left(\mathrm{HO}_{2}\right)$ radical, formic acid, nitric acid, and trans-sulfuric acid reactions obtained at the M06-2X/6-311++G(3df,3pd) level of theory. The black, white, blue, red, and yellow colors represent carbon, hydrogen, nitrogen, oxygen, and sulfur atoms, respectively. 

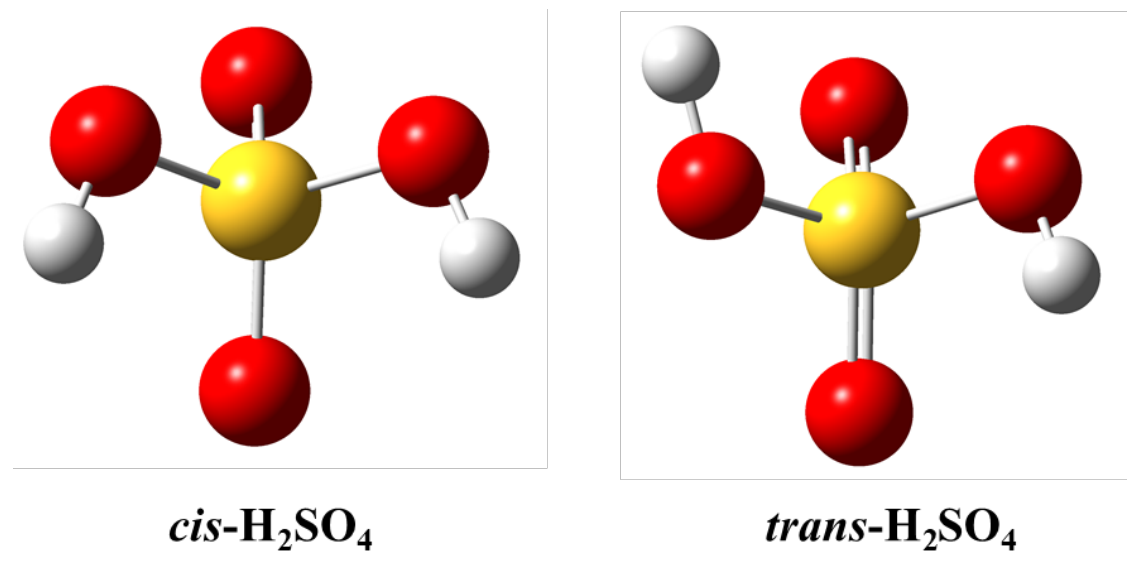

Figure S2. M06-2X/6-311++G(3df,3pd) level optimized geometries of two sulfuric acid conformers labeled as cis- $\mathrm{H}_{2} \mathrm{SO}_{4}$ and trans- $\mathrm{H}_{2} \mathrm{SO}_{4}$. The red, white, and yellow colors represent oxygen, hydrogen, and sulfur atoms, respectively. 


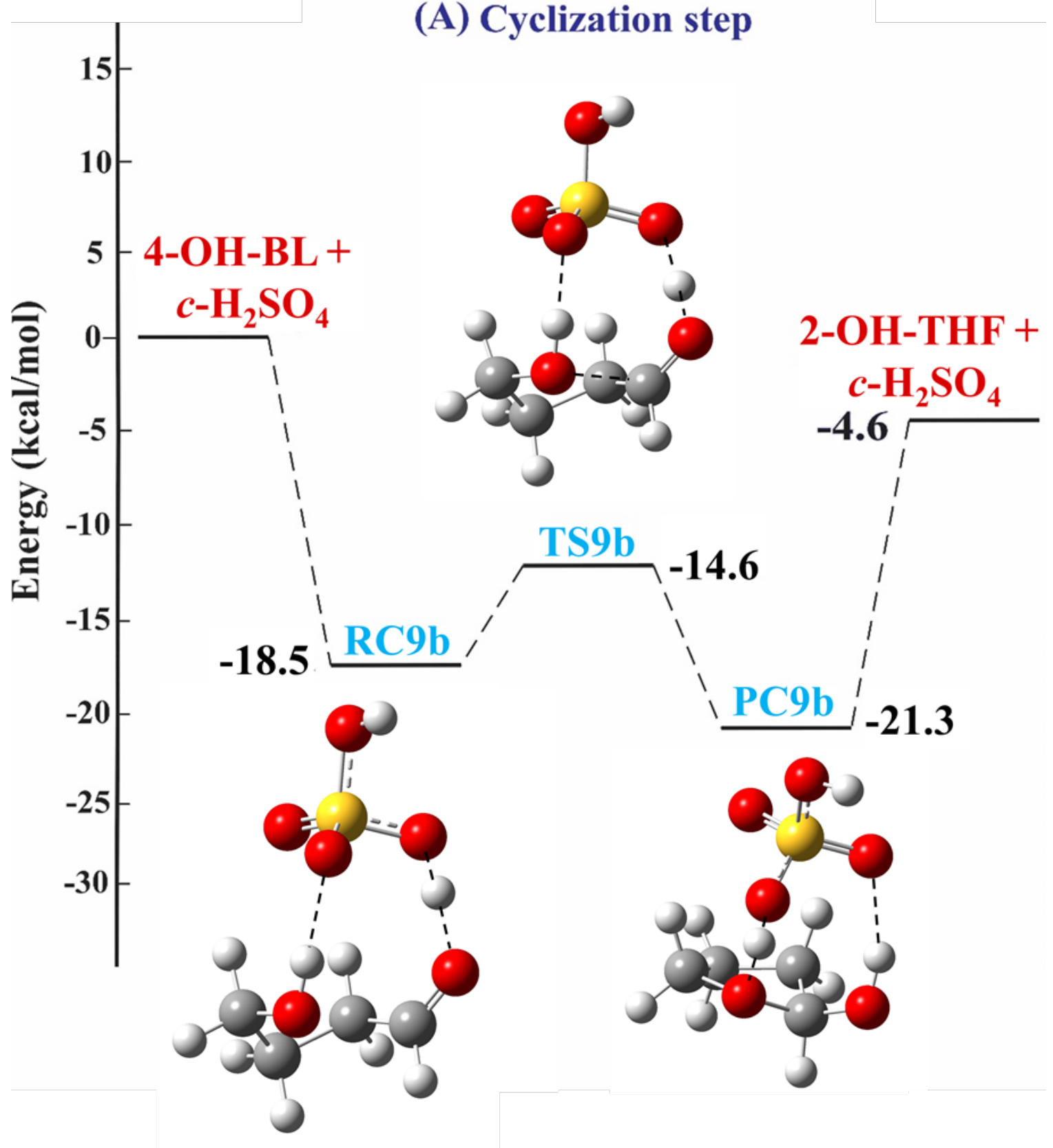

Figure S3. CCSD(T)/6-311++G(3df,3pd)//M06-2X/6-311++G(3df,3pd) calculated potential energy profile for the cyclization of 4-hydroxybutanal catalyzed by a single $c i s-\mathrm{H}_{2} \mathrm{SO}_{4}$ molecule (denoted as $c-\mathrm{H}_{2} \mathrm{SO}_{4}$ in the figure) leading to the formation of 2-hydroxytetrahydrofuran. The symbols correspond to: RC9b, TS9b, and PC9b represents reactant complex, transition state, and product complex, respectively. 


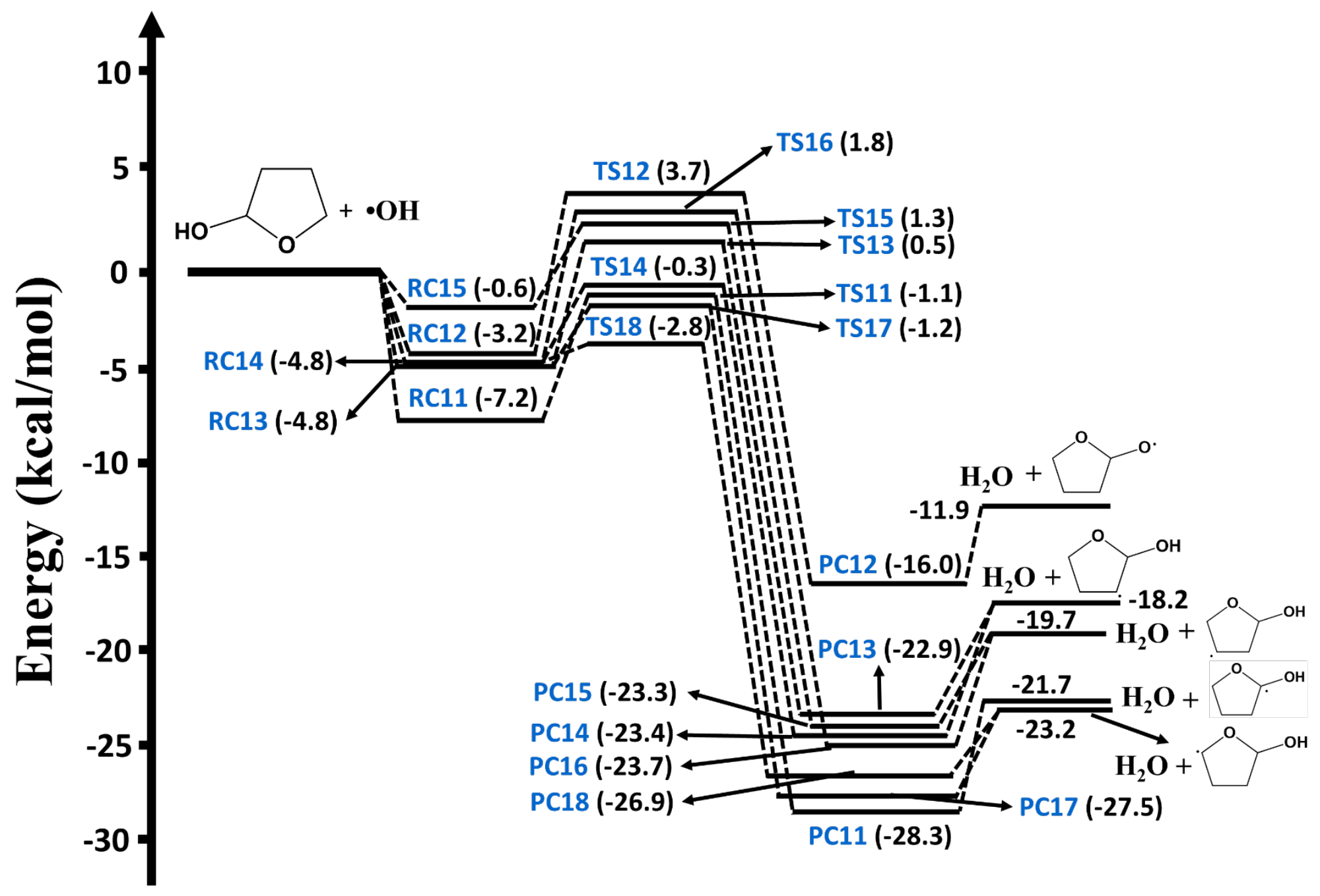

Figure S4: Potential energy diagram for the 2-OH-THF $+\cdot \mathrm{OH}$ reaction calculated at the CCSD(T)/6-311++G(3df,3pd)//M06-2X/6-311++G(3df,3pd) level. The energies (in kcal/mol) correspond to the abstraction of a hydrogen atom by the $\mathrm{OH}$ radical from the three $\mathrm{CH}_{2}$, one $\mathrm{CH}(\mathrm{OH})$ groups. The symbols RCn $(\mathrm{n}=11-15)$ correspond to the reactant complexes, $\mathrm{TSn}(\mathrm{n}=$ 11-18) correspond to the transition states, and PCn $(n=11-18)$ correspond to the product complexes, respectively. 